\title{
Vestibular schwannomas : new developments in the management of vestibular schwannomas
}

Citation for published version (APA):

van de Langenberg, R. (2012). Vestibular schwannomas : new developments in the management of vestibular schwannomas. [Doctoral Thesis, Maastricht University]. Datawyse / Universitaire Pers Maastricht. https://doi.org/10.26481/dis.20121207rl

Document status and date:

Published: 01/01/2012

DOI:

10.26481/dis.20121207rl

Document Version:

Publisher's PDF, also known as Version of record

\section{Please check the document version of this publication:}

- A submitted manuscript is the version of the article upon submission and before peer-review. There can be important differences between the submitted version and the official published version of record.

People interested in the research are advised to contact the author for the final version of the publication, or visit the DOI to the publisher's website.

- The final author version and the galley proof are versions of the publication after peer review.

- The final published version features the final layout of the paper including the volume, issue and page numbers.

Link to publication

\footnotetext{
General rights rights.

- You may freely distribute the URL identifying the publication in the public portal. please follow below link for the End User Agreement:

www.umlib.nl/taverne-license

Take down policy

If you believe that this document breaches copyright please contact us at:

repository@maastrichtuniversity.nl

providing details and we will investigate your claim.
}

Copyright and moral rights for the publications made accessible in the public portal are retained by the authors and/or other copyright owners and it is a condition of accessing publications that users recognise and abide by the legal requirements associated with these

- Users may download and print one copy of any publication from the public portal for the purpose of private study or research.

- You may not further distribute the material or use it for any profit-making activity or commercial gain

If the publication is distributed under the terms of Article $25 \mathrm{fa}$ of the Dutch Copyright Act, indicated by the "Taverne" license above, 


\section{Vestibular Schwannomas}

New developments in the management of vestibular schwannomas 
(C) Copyright R. van de Langenberg, Maastricht 2012

Universitaire Pers Maastricht

\section{ISBN 9789461591807}

\section{Cover design: Geert van de Langenberg}

Layout: Tiny Wouters

All rights reserved. No part of this publication may be reproduced in any form

or by any means, electronically, by print or otherwise without written permission of the copyright owner.

Financial support for the publication of this thesis was kindly provided by:

F BRAINLAB, ALK- Abelló, ATOS Medical, Beter Horen B.V., Carl Zeiss, Chiesi Pharmaceuticals B.V., Daleco Pharma B.V., Dos Medical B.V., Elekta, Electro Medical instruments B.V., Entermed, GSK Glaxo Smith Kline, HAL Allergy Benelux B.V., Oticon B.V., Pentax Lifecare Nederland B.V., Specsavers Hoorservice, Stallergenes B.V., Streukens Hooroplossingen, van Boxtel hoorwinkels, Nationale Hoorstichting/VriendenLoterij. 


\title{
Vestibular Schwannomas
}

\section{New developments in the management of vestibular schwannomas}

\author{
PROEFSCHRIFT \\ ter verkrijging van de graad van doctor \\ aan de Universiteit Maastricht, \\ op gezag van de Rector Magnificus, Prof. dr. L.L.G. Soete, \\ volgens het besluit van het College van Decanen, \\ in het openbaar te verdedigen \\ op vrijdag 7 december 2012 om 14:00 uur
}

door

Rick van de Langenberg Geboren op 25 oktober 1983 te Tilburg

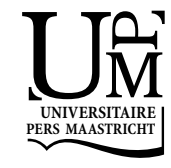




\section{Promotores}

Prof. dr. R.J. Stokroos

Prof. dr. B. Kremer

\section{Beoordelingscommissie}

Prof. dr. H. Kingma (voorzitter)

Prof. dr. ir. J.H.M. Frijns (Leids Universitair Medisch Centrum)

Prof. dr. W. Grolman (Universitair Medisch Centrum Utrecht)

Prof. dr. Y. Temel 


\section{Contents}

Chapter 1 General introduction and outline of the thesis

Chapter 2 Follow-up assessment of vestibular schwannomas: volume 25 quantification versus two-dimensional measurements Neuroradiology. 2009;51:517-24

Chapter 3 Predictors of volumetric growth and auditory deterioration 41 in vestibular schwannomas followed in a wait and scan policy Otology \& Neurotology. 2011;32:338-44

Chapter 4 Volume changes after stereotactic LINAC radiotherapy in 57 vestibular schwannoma: control rate and growth patterns International Journal Radiation Oncology * Biology * Physics. 2012;84:343-49

Chapter 5 Management of large vestibular schwannoma. Part I. Planned subtotal resection followed by Gamma Knife surgery: radiological and clinical aspects Journal of Neurosurgery. 2011;115:875-84

Chapter 6 Management of large vestibular schwannoma. Part II. Primary Gamma Knife surgery: radiological and clinical aspects

Journal of Neurosurgery. 2011;115:885-93

Chapter 7 Editorial and response to editorial. Vestibular schwannomas 115 Journal of Neurosurgery. 2011;115:894-99

Chapter 8 Discussion and conclusion

Summary

Samenvatting

Dankwoord 159

Curriculum Vitae 165

List of publications 169 

GENERAL INTRODUCTION

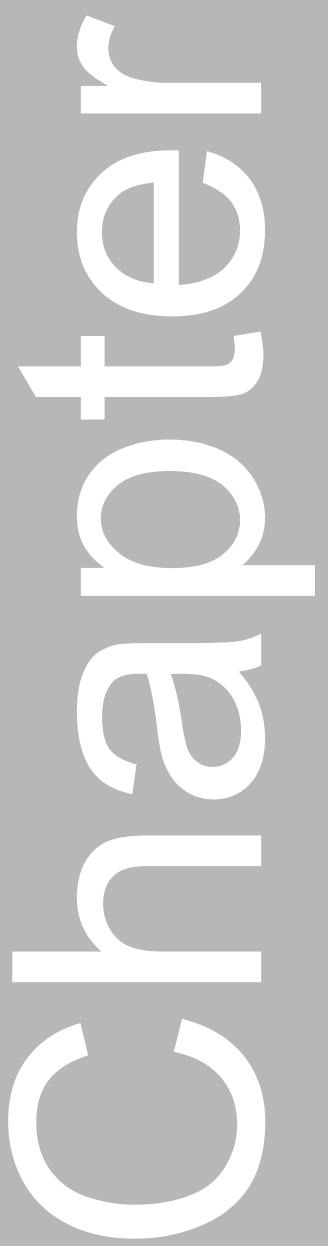


choment 


\section{General introduction}

Vestibular schwannomas (VS) are benign neoplasms originating from the Schwann cells of the neurolemnal sheath from the vestibular part of the 8th cranial nerve. When manifested in the internal auditory canal (IAC), they may grow in the direction of least mechanical resistance: the cerebellopontine angle (CPA). The vast majority of VS are sporadic and unilateral. In less than $5 \%$ of the cases, VS are bilateral and occur in the autosomal dominant inherited genetic condition called "neurofibromatosis type 2" (NF2), which is caused by inactivating mutations of the NF2 tumor suppressor gene. It is a relatively rare condition with an incidence of $1 / 50.000$ and almost all affected individuals develop bilateral VS by the age of $30^{1,2}$. The main focus of this thesis is on patients with unilateral VS.

VS comprise about $90 \%$ of all tumors appearing in the CPA, making it by far the most common neoplasm in this anatomic region ${ }^{3,4}$. In addition, $6 \%$ of all intracranial tumors consist of $V^{5,6}$. Because of it's delicate position, and proximity to several cranial nerves, VS can be accompanied by a large scale of symptoms, varying from sensorineural hearing loss to tinnitus, imbalance, vertigo, facial nerve paresis and trigeminal hypesthesia, ${ }^{7,8}$ Larger VS may cause hydrocephalus or symptomatic mass effect. The fact that vertigo is less prominent in VS patients is due to the fact that the ipsilateral central vestibular system gradually compensates for the slow decline in vestibular function on the affected side.

The annual incidence of diagnosed VS lies around 1/100.000 and is increasing due to an ageing population, and the increasing use and sensitivity of magnetic resonance imaging (MRI), being the most sensitive tool to diagnose these neoplasms $\mathrm{s}^{9,10}$. Before MRI was available, VS were diagnosed by performing $\mathrm{x}$-rays, later combined with pneumoventriculography ${ }^{11}$. Diagnoses could be made more accurately upon the introduction of the computed tomography (CT) scan. Diagnosis often did not occur, however, since a VS is isodense to surrounding brain tissue. The combination of CT with auditory brain stem responses (ABR), a fairly sensitive method to diagnose retrocochlear pathology, increased the possibility of accurate diagnosis. The use of iodated contrast material, which enhances the VS on a CT scan, also increased the sensitivity of diagnosis, although small VS $<5 \mathrm{~mm}$ were still frequently missed $^{12}$. All these imaging methods, which were used before the discovery of $\mathrm{MRI}$, are suboptimal techniques for visualizing VS. First, only a widening of the internal auditory canal can be ascertained by using plain x-rays or CT scan, although iodated contrasts may provide a more characteristic VS appearance. Second, specific VS features, as seen on MRI (specific enhancement pattern on gadolinium enhanced T1-weighted MRI and T2-weighted MRI), can not be seen with early imaging techniques, making it difficult to differentiate between a 
VS and, for example, a meningeoma, lipoma, epidermoid cyst or even a metastasis, which may also occur in the IAC or CPA ${ }^{13}$. Nowadays, CT imaging (the "second best" imaging method) in VS management is only used in patients with MRI contraindications, such as patients with intracranial clips, metal fragments or electric implants.

The true prevalence of VS probably is much higher than that diagnosed. Two large studies, performing 893 temporal bone dissections in cadavers, found a VS in $0.89 \%$ of the temporal bones ${ }^{14,15}$. The prevalence may therefore lie around 890/100.000, indicating that most of these tumors never become symptomatic and show no or very limited growth during life. This is the rationale for the "wait and scan policy" (W\&S), which has gained enormous popularity the last decades. It has therefore earned a clear position in the therapeutic arsenal for treating VS patients. Nowadays there are basically three main therapeutic options available for the management of VS: microsurgery, radiosurgery and the W\&S policy.

\section{The treatment triad of vestibular schwannomas}

\section{Microsurgery}

VS present themselves in a delicate space, within the posterior fossa, close to critical neurovascular structures, such as the brainstem and the cranial nerves. Historically, surgery of the CPA was a challenge, especially in absence of a good understanding of the microsurgical anatomy, asepsis, physiology, instruments and antibiotics.

The first description of a VS was most likely made in 1777 by Edouard Sandifort (1742-1814), a professor of anatomy in the Netherlands ${ }^{16}$. He discovered a small unilateral tumor "adherent to the right auditory nerve" during autopsy in a patient with right sided deafness ${ }^{6,16,17}$. Sir Charles Balance (18561936) is credited for the first successful VS surgery in a patient with progressive sensorineural hearing loss, ataxia and papilledema ${ }^{16,18}$ in 1894. As might be expected, the patients undergoing CPA surgery were suffering from severe symptoms due to processes causing symptomatic mass effect. Even though there was a complete fifth and seventh nerve paralysis postoperatively, the patient survived ${ }^{16,18}$. At that time most physicians found intracranial surgery unwise and irresponsible ${ }^{19}$. The mortality rates in VS surgery were high: at the international congress of medicine in London, in 1913, three leading European neurosurgeons, Horsley, Eiselberg and Krause, reported mortality rates varying from $67-84 \%$, and in case of survival, postoperative morbidity was high ${ }^{20}$. All these neurosurgeons used the retrosigmoid approach, in which they used an index finger to quickly dislodge the tumor which usually resulted in injury to the cerebellum and brainstem ${ }^{20}$. Harvey Cushing and later, Walter Dandy 
(Cushings pupil), two American neurosurgeons, further evolved the field of neurosurgery in the cerebellopontine angle by introducing refinements in the technique of removing the VS, thereby reducing postoperative mortality rates ${ }^{20}$. Cushing, for example, introduced the use of vascular clips and electrocautery, to control intraoperative hemorrhage ${ }^{21}$. In 1916, he published his results after treating patients with a "safe" subtotal resection, with postoperative mortality rates of $10-15 \%$. However, the mortality rate from recurrences was $54 \%$ in the 5 years follow-up period ${ }^{19}$. In contrast, Dandy favored a total extirpation. In 1941 he reported a mortality rate of $10.87 \%$, although facial nerve function was spared in only one of the 45 cases $^{22}$. Interestingly, discussion regarding these contrasting approaches toward VS (the extent of removal versus preservation of function in a benign tumor) is still actual, particularly in large VS and will be discussed in this thesis.

VS surgery continued to evolve, with the development of new surgical approaches, surgical and diagnostic aids and antibiotics. The retrosigmoid approach was used in the vast majority of VS surgery, until $1964^{16}$. William House, an otologist, and William Hitselberger, a neurosurgeon, introduced the operating microscope for VS microsurgery and developed the translabyrinthine and middle fossa approaches, as used today ${ }^{20,23}$. Their collaboration illustrated the onset of multidisciplinary evaluation and treatment of VS patients, which today is the rule rather than exception. These new approaches and techniques have led to an increased rate of facial nerve preservation, with at the same time a more radical removal of the tumor. In 1968, House reported a series of 200 surgically treated VS, with a facial nerve preservation rate of $68 \%$. Of the remaining $32 \%$ patients, $9.5 \%$ still showed improvement of function during follow-up ${ }^{24}$. The introduction of electromyography for monitoring the facial nerve intraoperatively, has further decreased the risk of facial nerve injury ${ }^{25}$.

Three main surgical techniques are presently used in the surgical treatment of VS, each with advantages and disadvantages (Table 1.1). The pro's and con's of any specific surgical technique should be weighed against other techniques (Table 1.1). Clearly, patient characteristics, such as hearing and contralateral hearing, vestibular function and VS characteristics and, in particular, VS size should be taken into account.

Hearing is classified according to the American Academy of OtolaryngologyHead and Neck Surgery (AAO-HNS) ${ }^{26}$. It is considered to be functional when it is graded as hearing class $A$ (pure tone audiogram (PTA) $\leq 30 \mathrm{~dB}$, speech discrimination score (SDS) $\geq 70 \%$ ) or B (PTA $>30 \mathrm{~dB}$, $\leq 50 \mathrm{~dB}$, SDS $\geq 50 \%$ ), whereas class C (PTA $>50 \mathrm{~dB}$, SDS $\geq 50 \%$ ) and D (PTA any level, SDS $<50 \%$ ) are not. This distinction is questionable. For example, for a patient with class $C$ hearing in the only hearing ear, the hearing is clearly functional.

If functional hearing is present, hearing preserving surgery can be performed. If a total resection is the final goal, the chance of hearing preservation is minimal 
when dealing with a VS $\geq 2 \mathrm{~cm}$, despite initial hearing status ${ }^{5,27}$. Using intraoperative $A B R$ may increase chances for hearing preservation ${ }^{28}$. The middle fossa approach seems to offer the best possibility for hearing preservation, due to the fact that only small VS can be operated by this approach: the chance of preserving hearing is inversely related to VS size ${ }^{29}$. Despite superb microsurgical skills and instruments, the mortality rate of VS surgery lies around $1 \%$.5, Moreover, postoperative morbidity should not be underestimated. First, there is the threat of complications, inherent to undergoing a major surgical procedure (such as pneumonia, deep vein thrombosis and specifically for cranial surgery, meningitis or cerebrospinal fluid leakage $)^{5}$. Second, morbidity to adjacent cranial nerves plays an important role. As is the case with the cochlear nerve in pursuing a complete resection, facial nerve outcome is also inversely related to the initial VS size ${ }^{31-33}$. Especially the effect of facial nerve paresis on the patient's quality of life should not be underrated $^{34}$.

Table $1.1^{5}$

\begin{tabular}{|c|c|c|}
\hline Approach & Advantage & Disadvantage \\
\hline Middle Fossa & $\begin{array}{l}\text { - Excellent exposure fundus IAC } \\
\text { and labyrinthine segment facial } \\
\text { nerve } \\
\text { - Auditory function may be } \\
\text { preserved }\end{array}$ & $\begin{array}{l}\text { - Not applicable for tumors } \\
\text { extending the IAC because of } \\
\text { poor exposure posterior fossa, } \\
\text { therefore only applicable for small } \\
\text { intracanalicular VS } \\
\text { - Required significant traction on } \\
\text { the temporal lobe with the risk of } \\
\text { seizures, aphasia and stroke } \\
\text { - The facial nerve is on top of the } \\
\text { VS and therefore more at risk }\end{array}$ \\
\hline Translabyrinthine & $\begin{array}{l}\text { - Excellent exposure fundus IAC } \\
\text { and labyrinthine segment facial } \\
\text { nerve } \\
\text { - Most direct working distance to } \\
\text { CPA } \\
\text { - Low or little cerebellar traction in } \\
\text { small tumors }\end{array}$ & $\begin{array}{l}\text { - Sacrifice of auditory and } \\
\text { vestibular function } \\
\text { - Limited exposure posterior fossa } \\
\text { - Relatively complex approach } \\
\text { - Variable exposure depending on } \\
\text { pneumatisation of the mastoid } \\
\text { - Limited exposure IAC possible in } \\
\text { case of high jugular bulb }\end{array}$ \\
\hline Retrosigmoid & $\begin{array}{l}\text { - Auditory function may be } \\
\text { preserved } \\
\text { - Excellent exposure posterior } \\
\text { fossa, cerebellum, brain stem. } \\
\text { - Relatively rapid approach }\end{array}$ & $\begin{array}{l}\text { - Difficult exposure to the fundus of } \\
\text { the IAC } \\
\text { - Oblique angle (long working } \\
\text { distance) } \\
\text { - Required retraction of cerebellum } \\
\text { - Higher incidence of cerebrospinal } \\
\text { fluid leak }\end{array}$ \\
\hline
\end{tabular}

IAC: internal auditory canal; VS: vestibular schwannoma; CPA: cerebellopotine angle. 


\section{Radiosurgery}

The main goal of radiosurgery is not, as is the case with malignant tumors, eradication of the tumor. The primary goal in VS radiosurgery is to achieve growth control. High energy radiation beams cause irreparable damage to cells by acting on essential cell structures (e.g. cell membranes and DNA). Generally, VS are being treated with either Gamma Knife surgery (GKS) or linear accelerator radiosurgery (LINAC). Both of these methods will be evaluated in this thesis.

\section{Gamma Knife surgery}

Professor Lars Leksell (1907-1986), a neurosurgeon of the Karolinska Institute in Stockholm, Sweden, developed the Leksell Gamma Knife in the 1960s. This technique facilitated ionizing radiation to specific pathological targets with the intention of inactivating the pathological substrate: with this system access could be gained to any intracranial region with minimal trauma. In 1969 Leksell described the use of GKS to treat $\mathrm{VS}^{35}$. The first computerized planning was devised in 1976 and given the limitations of CT imaging that time, one week planning time was required ${ }^{36}$.

The Gamma Knife works by using Co-60 (Cobalt) radiation sources, a radioactive isotope that emits a penetrating gamma-ray by decay. This is an energetic photon (from the same electromagnetic family as light or x-rays, but far more energetic and harmful). Gamma-rays are capable of damaging living cells when they slow down and transfer energy to the cells. In the latest version of the Gamma Knife device, the Leksell Gamma Knife Perfexion, 192 of these sources are available. The Co-60 sources are fitted and do not rotate around the patient. The individual beams are very narrow. This enables the construction of a very precise radiation field, focused on and limited to the concerned lesion. Normal tissue escapes dangerous levels of radiation, because there is a rapid fall of radiation levels just beyond the edge of the lesion $^{37}$. It is the surgical precision of the radiation field, administered in a single session, that led to the term radiosurgery ${ }^{38}$. "Stereotactic" means that it is possible to locate an intracerebral structure. To gain a high degree of "stereotactic precision" the patient's head is fixed with a stereotactic frame. Pretreatment planning MRI occurs with this head-frame on. By using specific software, physicians can localize the intracranial pathology on MRI and plan the radiosurgical procedure.

\section{LINAC radiosurgery}

Another radiosurgical approach is to treat VS with LINAC radiosurgery. In 1951, Lars Leksell was experimenting with "radiosurgery" by using an x-ray 
unit. The limited penetration of these x-rays (which are also photons), resulted in suboptimal doses spread across the treated brain ${ }^{39,40}$. Leksell therefore sought a more penetrating beam and found Co-60, used for $\mathrm{GKS}^{39}$. In 1983 Betti and Derechinsky described the use of a hospital based linear accelerator for radiosurgery ${ }^{41}$. Throughout the 1980s several LINAC based radiosurgery systems were described ${ }^{39}$. LINAC based radiosurgery still occurs using $x$-rays, which were not suitable for intracranial radiosurgery, decades earlier. The system works as follows: to produce photons, electrons are released from an electron gun and injected into an accelerator tube. This tube consists of several chambers where electrical charges can be alternated quickly from positive to negative. When the negatively charged electron exits the tube, they can be focused with a magnetic field. These "bending magnets" are located at the head of the LINAC station. The electrons are, after bending, fired on a metal target, creating photons with the "Brehmstrahlungseffect" (electromagnetic radiation is produced by deceleration of a charged particle when deflected by another particle, in this case an electron and an atomic nucleus (metal target)). The electron loses kinetic energy which is converted into photons. The photon beam is then collimated and focused on a stereotactically defined intracranial target $^{39}$. A major difference, when compared with GKS, is that the radiation beams are emitted by a single source, rotating slowly around the head of the patient.

An advantage of LINAC radiosurgery is that it is possible to apportion the dose to be given to the intracranial process requiring irradiation. This is then called "fractionated radiotherapy": patients need to be treated day after day, for several weeks, which is clearly not optional for all patients because of traveling distance or comorbidity. Evidence indicates that fractionated LINAC radiotherapy might be more appropriate in case of functional hearing, and the wish to preserve this, with similar tumor control rates ${ }^{42}$.

\section{Radiosurgical control rates and morbidity}

In the last decades, considerable attention has been paid to the radiosurgical dose administered to VS. In the early days of VS radiosurgery, it was assumed that these benign VS were relatively insensitive to radiation, and therefore a dose to the tumor periphery of 25-35 Gy was used ${ }^{43}$. Furthermore, planning schedules were less reliable because physicians were using CT scans to calculate the area of dose coverage. The dose was gradually lowered, and MRI was introduced, making it possible to make a far more reliable radiosurgical planning than was the case with CT planning. In 1992 Linskey et al. reported results after administering $16 \mathrm{~Gy}$ to the VS margins ${ }^{44}$ : a high rate of tumor control was seen $(97 \%)$, however $30 \%$ of the patients developed a facial nerve paresis in the first postradiosurgical year and 33\% developed trigeminal neuropathies. Although the vast majority of these pareses improved over time, 
it was assumed that the optimal equilibrium of growth control versus preservation of function had not yet been established. In the past ten years it seems that an optimal balance has been found: the marginal dose applied to the VS has been minimized to 12-13 Gy: a similar growth control is achieved, with lower postradiosurgical morbidity: facial nerve paresis and trigeminal dysfunction are seldom reported, namely in $\leq 1 \%$ of the cases ${ }^{45-48}$. This holds true for both GKS and LINAC radiosurgery. Risk factors for facial and trigeminal nerve dysfunction after radiosurgery are the prescribed dose and volume of the $\mathrm{VS}^{49-51}$. Apart from excellent results regarding growth control, hearing preservation is possible ${ }^{27}$. Advantages of a radiosurgical treatment are the avoidance of surgery with a surgically related hospital stay, morbidity and mortality. In addition, radiosurgery is less expensive than a microsurgical resection provided that the rate of tumor progression after radiosurgery remains low in the long-term follow-up ${ }^{52}$.

Two important disadvantages remain in treating VS with radiosurgery. First, the VS does not disappear, necessitating lifelong follow-up. Second, there is a risk of inducing a secondary, radiotherapy induced malignancy. The incidence varies in literature from $1 / 1000$ until $3 / 200.000$ of the treated patients ${ }^{53,54}$.

\section{Wait and scan policy}

The indolent nature of VS, in which many tumors never grow, or grow slow enough to never require treatment, has led to the introduction of the W\&S policy. In these patients it is unnecessary to perform an intervention which is, in spite of all improvements, not without risks. Also the fact that more small (asymptomatic) intracanalicular VS are being diagnosed contributed to the popularity of the W\&S policy, in which patients with smaller VS are entered ${ }^{10,55}$ Patients may also enter the W\&S policy because of an explicit wish not to undergo treatment, (severe) comorbidity, older age, or the presence of a VS in the only hearing ear.

When a patient enters this W\&S policy, they are followed by sequential MRI, clinical evaluation and audiometric testing if functional hearing is present. After the diagnosis is made, the following MRI will take place after 6 months and annually thereafter. In case of minimal or no growth, MR imaging every 2 years is possible. It is important to inform patients about the pro's and con's of this conservative way of following the VS: because of the indolent nature of VS, intervention, with possible morbidity or even mortality, might be prevented. On the other hand, in case of tumor growth during a W\&S policy, a VS may become more difficult to operate upon or functional hearing, if present, may be lost. Furthermore, radiological and clinical follow-up for the rest of the patient's life is required. A W\&S policy is also interesting from a cost-effective perspective: the W\&S policy offers an economic advantage when compared with invasive treatment options ${ }^{56}$. This argument plays an important role in the 
light of the increasing number of patients being diagnosed with VS, and with the health care costs, that are rising annually.

Growth, retaining functional hearing and absence of severe symptoms are important factors for VS patients being followed in a W\&S policy. Changes in these factors may cause a switch in treatment policy, from conservative to invasive. Growth can be estimated by measuring various dimensions. Because the W\&S policy is based on evaluating the presence of growth, a precise method for measuring VS size, is essential. Historically, VS are measured with two-dimensional measurements, and still, in clinical practice, clinicians use an arbitrary difference of 1 or 2 millimeter between subsequent scans in one or two directions as proof of significant growth. If one takes into account that a VS is not a flat object, but a three-dimensional process, volume measurements may provide a better, "real", estimate of VS size. Invasive treatment decisions would then be based on a more precise measurement. This issue is an important topic of this thesis.

Considerable research has been directed towards finding risk factors for growth and preserving functional hearing. Many studies found factors predicting growth or hearing deterioration, although these findings are not consistent among studies. In this thesis, baseline risk factors predicting growth or hearing deterioration for VS patients being followed in a W\&S policy will be discussed. A large meta-analysis, analyzing all studies publishing results on predictive factors for growth and hearing deterioration during a W\&S policy until 2005, found growth during the first year to predict future growth. In addition, during follow-up time, $50 \%$ of the patients lost functional hearing ${ }^{57}$. Patients with excellent results on both tone and speech audiogram $(100 \%$ speech discrimination score (SDS)) on the affected ear, are somehow "protected" from losing serviceable hearing during follow-up in a W\&S policy, according to a large study of Stangerup et al. ${ }^{58}$. Serviceable hearing was retained by $90 \%$ of these patients during follow-up. This percentage decreased significantly when initial SDS was less than $100 \%$.

\section{Therapy selection}

In VS literature, VS are often divided into 4 grades, according to the Koos classification $^{59}$. Koos Grade I VS are located in the IAC. Grade II extends into the CPA; Grade III compresses the brain stem and grade IV deviates the $4^{\text {th }}$ ventricle. For progression in small/medium sized VS (typically Koos Grade I, II, sporadically III) radiosurgery is the main treatment option. Two prospective, non randomized studies have shown comparable outcomes for small/medium sized VS after radiosurgery and microsurgery, with a higher chance of hearing preservation and avoiding cranial nerve morbidity and mortality in the radiosurgical group ${ }^{60,61}$. In case of progression after radiosurgery, a second 
radiosurgical treatment is possible, with good control rates and a very low risk of complications ${ }^{62,63}$.

Progressive Koos Grade III and IV VS can still be treated with radiosurgery, if the extracanalicular dimension is $<4 \mathrm{~cm}$, in absence of symptomatic mass effect $^{64}$. Larger VS $(>4 \mathrm{~cm})$ are not considered as good candidates for radiosurgery, because some degree of symptomatic mass effect is usually present. In case of symptomatic mass effect, a microsurgical treatment is the therapy of choice. In this thesis results are presented of large VS being treated either by subtotal microsurgical resection, followed by radiosurgery or by primary radiosurgery. This group of large VS represents the "grey" area in which indications for one therapy or another are not always clear cut.

In case of continuing VS growth or severe symptomatology, the need for intervention is evident. The presence of serviceable hearing, and the wish to preserve this, seems less evident. As the meta-analysis of Smouha et al. indicates, the overall risk of losing functional hearing lies around $50 \%$, when a patient is being followed in a W\&S policy ${ }^{57}$. The possible causes of hearing deterioration in VS patients in a W\&S policy will be discussed in this thesis. A recent systematic review of Yang et al. showed a hearing preservation rate of $57 \%$ after radiosurgical VS treatment. Initial VS volume did not affect hearing outcome while the radiosurgical dose used $\mathrm{did}^{65}$. Wanibuchi et al. reviewed results of larger studies preserving functional hearing in VS microsurgery, which ranged from $18-58 \%{ }^{27}$; in large VS the range varied from $0-28 \%$. Generally, the highest chance of preserving functional hearing is in the case of small intracanalicular VS, operated with the middle fossa approach ${ }^{29}$. A problem regarding hearing outcome after complete microsurgical resection is that patients with postoperative functional hearing may subsequently experience hearing decline. Chee et al. demonstrated this in their study. They followed patients in whom hearing preserving VS microsurgery was performed. Forty percent of the patients with functional hearing in the early postoperative period, lost it during long term follow-up, with a mean follow-up of 9.4 years $^{66}$. Possible hypotheses are that postoperative ischemia results from scarring, fibrosis compresses the vessels in the internal auditory canal or a VS recurrence occurs that infiltrates the cochlear nerve ${ }^{29}$. Late loss of functional hearing after radiosurgery occurs because of late toxic effects of the dose applied to the cochlea. Continuing compression of VS on the cochlear nerve may also play a role. According to the above, it seems that when intervention is necessary in progressive large VS, a radiosurgical approach should be performed if there is a focus on preserving hearing. In smaller, non-growing VS with a superb SDS, a W\&S policy may be advised ${ }^{58}$. In other cases, it is difficult to determine the superior treatment regimen with the best chance of hearing preservation. This is mainly due to the lack of prospective, randomized controlled trials. The two mentioned prospective, non randomized studies found 
better hearing after radiosurgery, when compared to microsurgery, however, the middle fossa approach (giving the best hearing results for small intracanalicular VS) was not practiced in one study ${ }^{60}$, and in only $6 \%$ of the patients in the other ${ }^{61}$.

The absence of prospective randomized studies does not hold true for just the hearing outcome in VS management, but also for factors such as VS control rates or other clinical features, for all three treatment modalities. Because it is difficult to conduct such studies, answers are not expected in the future. It seems that any evidence we have so far, is the best we will have. Apart from using results from VS literature, the decision to undergo a specific treatment certainly also depends on the expertise and VS exposure of each multidisciplinary skull base team (including a neurotologists, neurosurgeon, radiotherapist and neuroradiologist). For example, the results of VS microsurgery show a learning curve indicating that the preservation of functional hearing or facial nerve function also depend on the experience of the operating surgeon $^{67,68}$. 


\section{Aims and outline of the present thesis}

The aim of this thesis was to gain insight in new developments for managing VS, both conservatively and invasively. As discussed in the current chapter, the management of VS has evolved tremendously during the past century: emphasis in the early years was on saving lives, nowadays it is on saving function. In the present thesis, this evolution is being continued, by comparing current "common practices" with new ones, in order to define new guidelines in the management of VS patients.

Chapter 2 addresses a key issue in VS management: the evaluation of VS growth. So far, in clinical practice, VS are measured by using two-dimensional measurements. This chapter provides evidence for the use of volume measurements in VS management. Furthermore, cut off points for growth beyond measurement error, both for two-dimensional and volume measurements are given. These data provide the cornerstone for the chapters following.

Chapter 3 evaluates baseline risk factors predicting volumetric growth or hearing deterioration in patients with VS, being followed in a W\&S policy.

Chapter 4 presents the outcome (the radiological control rate and the absence of further intervention rate) of small/medium sized VS, being treated with LINAC based stereotactic radiosurgery or radiotherapy.

Chapter 5 presents the results of patients with large VS, treated with a planned subtotal microsurgical resection, in order to decompress the brain stem, followed by GKS to obtain growth control of the residual VS.

Chapter 6 presents the results of patients with large VS, being treated with primary GKS, without previous treatment.

Chapter 7 presents an editorial of experts in the field of VS management on Chapter 5 and 6 , with a response to this editorial. This chapter emphasizes the shifting borders of radiosurgery and the "grey" area of VS management in large VS, in which exact indications for one treatment or another are not always clear cut. For various reasons, physicians may therefore opt for different treatment strategies.

Chapter 8 comprises the discussion and conclusion.

Chapter 9 includes a summary of this thesis. 


\section{References}

1. Goutagny S, Kalamarides M. Meningiomas and neurofibromatosis. J Neurooncol. 2010;99:341-7.

2. Evans DG. Neurofibromatosis 2 [Bilateral acoustic neurofibromatosis, central neurofibromatosis, NF2, neurofibromatosis type II]. Genet Med. 2009;11:599-610.

3. Kohan D, Downey LL, Lim J, Cohen NL, Elowitz E. Uncommon lesions presenting as tumors of the internal auditory canal and cerebellopontine angle. Am J Otol. 1997;18:386-92.

4. Bonneville F, Sarrazin JL, Marsot-Dupuch K, Iffenecker C, Cordoliani YS, Doyon D, Bonneville JF. Unusual lesions of the cerebellopontine angle: a segmental approach. Radiographics. 2001;21:419-38.

5. Bennett M, Haynes DS. Surgical approaches and complications in the removal of vestibular schwannomas. Otolaryngol Clin North Am. 2007;40:589-609, ix-x.

6. Machinis TG, Fountas KN, Dimopoulos V, Robinson JS. History of acoustic neurinoma surgery. Neurosurg Focus. 2005;18:e9.

7. Rosenberg SI. Natural history of acoustic neuromas. Laryngoscope. 2000;110: 497-508.

8. Walsh RM, Bath AP, Bance ML, Keller A, Tator $\mathrm{CH}$, Rutka JA. The role of conservative management of vestibular schwannomas. Clin Otolaryngol Allied Sci. 2000;25:28-39.

9. Shin YJ, Fraysse B, Cognard C, Gafsi I, Charlet JP, Berges C, Deguine O, Tremoulet M. Effectiveness of conservative management of acoustic neuromas. Am J Otol. 2000;21:857-62.

10. Stangerup SE, Caye-Thomasen $P$, Tos $M$, Thomsen J. The natural history of vestibular schwannoma. Otol Neurotol. 2006;27:547-52.

11. Dandy WE. Ventriculography Following the Injection of Air into the Cerebral Ventricles. Ann Surg. 1918;68:5-11.

12. Mafee MF, Valvassori GE. Imaging of the temporal bone. In: Wackym PA, Snow JB (eds) Ballenger's Otorhinolaryngology Head and Neck Surgery. Shelton CT, USA: Peoples Medical Publishing House. 2009:145-72.

13. van de Langenberg $R$, Stokroos $R$, de Bondt BJ. Radiology quiz case 2. Metastasis from NSCLC in both IACs. Arch Otolaryngol Head Neck Surg. 2009;135:717, 9.

14. Leonard JR, Talbot ML. Asymptomatic acoustic neurilemoma. Arch Otolaryngol. 1970;91:117-24.

15. Stewart TJ, Liland J, Schuknecht HF. Occult schwannomas of the vestibular nerve. Arch Otolaryngol. 1975;101:91-5.

16. Koerbel A, Gharabaghi A, Safavi-Abbasi S, Tatagiba M, Samii M. Evolution of vestibular schwannoma surgery: the long journey to current success. Neurosurg Focus. 2005;18:e10.

17. Ahn MS, Jackler RK, Lustig LR. The early history of the neurofibromatosis. Evolution of the concept of neurofibromatosis type 2. Arch Otolaryngol Head Neck Surg. 1996;122:1240-9.

18. Stone JL. Sir Charles Ballance: pioneer British neurological surgeon. Neurosurgery. 1999;44:610-31.

19. Cushing $H$. Tumors of the Nervus Acusticus and the Syndrome of the Cerebellopontine Angle. Philadelphia: Saunders; 1917.

20. Akard W, Tubbs RS, Seymour ZA, Hitselberger WE, Cohen-Gadol AA. Evolution of techniques for the resection of vestibular schwannomas: from saving life to saving function. J Neurosurg. 2009;110:642-7. 
21. Ellis H. Harvey Cushing and electrosurgery. Contemp Surg. 1981;19:73-5.

22. Dandy WE. Results of removal of acoustic tumors by the unilateral approach Arch Surg. 1941;42:1026-33.

23. House WF. Surgical exposure of the internal auditory canal and its contents through the middle, cranial fossa. Laryngoscope. 1961;71:1363-85.

24. House WF. Acoustic neuroma. Case summaries. Arch Otolaryngol. 1968;88: 586-91.

25. Youssef AS, Downes AE. Intraoperative neurophysiological monitoring in vestibular schwannoma surgery: advances and clinical implications. Neurosurg Focus. 2009;27:E9.

26. Committee on Hearing and Equilibrium guidelines for the evaluation of hearing preservation in acoustic neuroma (vestibular schwannoma). American Academy of Otolaryngology-Head and Neck Surgery Foundation, INC. Otolaryngol Head Neck Surg. 1995;113:179-80.

27. Wanibuchi M, Fukushima T, McElveen JT, Jr., Friedman AH. Hearing preservation in surgery for large vestibular schwannomas. J Neurosurg. 2009;111:845-54.

28. Phillips DJ, Kobylarz EJ, De Peralta ET, Stieg PE, Selesnick SH. Predictive factors of hearing preservation after surgical resection of small vestibular schwannomas. Otol Neurotol. 2010;31:1463-8.

29. Noudel R, Gomis P, Duntze J, Marnet D, Bazin A, Roche PH. Hearing preservation and facial nerve function after microsurgery for intracanalicular vestibular schwannomas: comparison of middle fossa and retrosigmoid approaches. Acta Neurochir (Wien). 2009;151:935-44.

30. Samii M, Matthies C. Management of 1000 vestibular schwannomas (acoustic neuromas): surgical management and results with an emphasis on complications and how to avoid them. Neurosurgery. 1997;40:11-21.

31. Wiet RJ, Mamikoglu B, Odom L, Hoistad DL. Long-term results of the first 500 cases of acoustic neuroma surgery. Otolaryngol Head Neck Surg. 2001;124: 645-51.

32. Mamikoglu B, Wiet RJ, Esquivel CR. Translabyrinthine approach for the management of large and giant vestibular schwannomas. Otol Neurotol. 2002;23: 224-7.

33. Lanman TH, Brackmann DE, Hitselberger WE, Subin B. Report of 190 consecutive cases of large acoustic tumors (vestibular schwannoma) removed via the translabyrinthine approach. J Neurosurg. 1999;90:617-23.

34. Cross T, Sheard CE, Garrud P, Nikolopoulos TP, O'Donoghue GM. Impact of facial paralysis on patients with acoustic neuroma. Laryngoscope. 2000;110:1539-42.

35. Leksell L. A note on the treatment of acoustic tumours. Acta Chir Scand. 1971;137:763-5.

36. Bambakidis NC, Megerian CA, Spetzler RF. Surgery of the Cerebellopontine Angle Shelton, CT, USA: Peoples Medical Publishing House. 2009:135.

37. Griffith R, Harsh IV. Gamma Knife Radiosurgery: principles and techniques. In: Germano IM, ed. LINAC and Gamma Knife Radiosurgery. Park Ridge, III, USA: The American Association of Neurological Surgeons. 1999:11-8.

38. Ganz JC. Gamma Knife Neurosurgery. Vienna, Austria. Springer-Verlag. 2011:5.

39. Schultz CJ, Gillin M, Mueller WM. Modified Linear Accelerator Radiosurgery: Principles and Techniques. In: Germano IM, ed. LINAC and Gamma Knife Radiosurgery. Park Ridge, III, USA: The American Association of Neurological Surgeons. 1999:19-30. 
40. Leksell L. The stereotaxic method and radiosurgery of the brain. Acta Chir Scand. 1951;102:316-9.

41. Betti O, Derechinsky V. [Multiple-beam stereotaxic irradiation]. Neurochirurgie. 1983;29:295-8.

42. Collen C, Ampe B, Gevaert T, Moens M, Linthout N, De Ridder M, Verellen D, D'Haens J, Storme G. Single Fraction Versus Fractionated Linac-Based Stereotactic Radiotherapy for Vestibular Schwannoma: A Single-Institution Experience. Int J Radiat Oncol Biol Phys. 2011, Jun 11 [Epub ahead of print].

43. Iwai $\mathrm{Y}$, Yamanaka K, Shiotani M, Uyama T. Radiosurgery for acoustic neuromas: results of low-dose treatment. Neurosurgery. 2003;53:282-87.

44. Lunsford LD, Linskey ME. Stereotactic radiosurgery in the treatment of patients with acoustic tumors. Otolaryngol Clin North Am. 1992;25:471-91.

45. Lunsford LD, Niranjan A, Flickinger JC, Maitz A, Kondziolka D. Radiosurgery of vestibular schwannomas: summary of experience in 829 cases. J Neurosurg. 2005;102 Suppl:195-9.

46. Chung WY, Liu KD, Shiau CY, Wu HM, Wang LW, Guo WY, Ho DM, Pan DH. Gamma knife surgery for vestibular schwannoma: 10-year experience of 195 cases. J Neurosurg. 2005;102 Suppl:87-96.

47. Kondziolka D, Lunsford LD, McLaughlin MR, Flickinger JC. Long-term outcomes after radiosurgery for acoustic neuromas. N Engl J Med. 1998;339:1426-33.

48. Friedman WA, Bradshaw $P$, Myers A, Bova FJ. Linear accelerator radiosurgery for vestibular schwannomas. J Neurosurg. 2006;105:657-61.

49. Flickinger JC, Kondziolka D, Niranjan A, Maitz A, Voynov G, Lunsford LD. Acoustic neuroma radiosurgery with marginal tumor doses of 12 to $13 \mathrm{~Gy}$. Int J Radiat Oncol Biol Phys. 2004;60:225-30.

50. Ito K, Shin M, Matsuzaki M, Sugasawa K, Sasaki T. Risk factors for neurological complications after acoustic neurinoma radiosurgery: refinement from further experiences. Int J Radiat Oncol Biol Phys. 2000;48:75-80.

51. Williams JA. Fractionated stereotactic radiotherapy for acoustic neuromas: preservation of function versus size. J Clin Neurosci. 2003;10:48-52.

52. Banerjee R, Moriarty JP, Foote RL, Pollock BE. Comparison of the surgical and follow-up costs associated with microsurgical resection and stereotactic radiosurgery for vestibular schwannoma. J Neurosurg. 2008;108:1220-4.

53. Loeffler JS, Niemierko A, Chapman PH. Second tumors after radiosurgery: tip of the iceberg or a bump in the road? Neurosurgery. 2003;52:1436-40.

54. Ganz JC. Gamma knife radiosurgery and its possible relationship to malignancy: a review. J Neurosurg. 2002;97(5 Suppl):644-52.

55. Stangerup SE, Caye-Thomasen $\mathrm{P}$, Tos M, Thomsen J. Change in hearing during 'wait and scan' management of patients with vestibular schwannoma. J Laryngol Otol. 2008;122:673-81.

56. Verma S, Anthony R, Tsai V, Taplin M, Rutka J. Evaluation of cost effectiveness for conservative and active management strategies for acoustic neuroma. Clin Otolaryngol. 2009;34:438-46.

57. Smouha EE, Yoo M, Mohr K, Davis RP. Conservative management of acoustic neuroma: a meta-analysis and proposed treatment algorithm. Laryngoscope. 2005; 115:450-4.

58. Stangerup SE, Tos M, Thomsen J, Caye-Thomasen P. Hearing outcomes of vestibular schwannoma patients managed with 'wait and scan': predictive value of hearing level at diagnosis. J Laryngol Otol. 2010;124:490-4. 
59. Koos WT, Day JD, Matula C, Levy DI. Neurotopographic considerations in the microsurgical treatment of small acoustic neurinomas. J Neurosurg. 1998;88: 506-12.

60. Myrseth E, Moller P, Pedersen PH, Lund-Johansen M. Vestibular schwannoma: surgery or gamma knife radiosurgery? A prospective, nonrandomized study. Neurosurgery. 2009;64:654-61.

61. Pollock BE, Driscoll CL, Foote RL, Link MJ, Gorman DA, Bauch CD, Mandrekar $\mathrm{JN}$, Krecke $\mathrm{KN}$, Johnson $\mathrm{CH}$. Patient outcomes after vestibular schwannoma management: a prospective comparison of microsurgical resection and stereotactic radiosurgery. Neurosurgery. 2006;59:77-85.

62. Yomo S, Arkha Y, Delsanti C, Roche PH, Thomassin JM, Regis J. Repeat gamma knife surgery for regrowth of vestibular schwannomas. Neurosurgery. 2009;64: 48-54.

63. Liscak R, Vladyka V, Urgosik D, Simonova G, Vymazal J. Repeated treatment of vestibular schwannomas after gamma knife radiosurgery. Acta Neurochir (Wien). 2009;151:317-24.

64. Yang HC, Kano H, Awan NR, Lunsford LD, Niranjan A, Flickinger JC, Novotny J, Jr., Bhatnagar JP, Kondziolka D. Gamma Knife radiosurgery for larger-volume vestibular schwannomas. Clinical article. J Neurosurg. 2011;114:801-7.

65. Yang I, Aranda D, Han SJ, Chennupati S, Sughrue ME, Cheung SW, Pitts LH, Parsa AT. Hearing preservation after stereotactic radiosurgery for vestibular schwannoma: a systematic review. J Clin Neurosci. 2009;16:742-7.

66. Chee GH, Nedzelski JM, Rowed D. Acoustic neuroma surgery: the results of longterm hearing preservation. Otol Neurotol. 2003;24:672-6.

67. Mangham CA, Jr. Retrosigmoid versus middle fossa surgery for small vestibular schwannomas. Laryngoscope. 2004;114:1455-61.

68. Moffat DA, Hardy DG, Grey PL, Baguley DM. The operative learning curve and its effect on facial nerve outcome in vestibular schwannoma surgery. Am J Otol. 1996; 17:643-7. 



\section{FOLLOW-UP ASSESSMENT OF VESTIBULAR SCHWANNOMAS: VOLUME QUANTIFICATION VERSUS TWO-DIMENSIONAL MEASUREMENTS}

Rick van de Langenberg Bert J de Bondt Patty J Nelemans Brigitta G Baumert Robert J Stokroos

Neuroradiology. 2009;51:517-524 


\section{Abstract}

\section{Introduction}

A conservative treatment strategy is often proposed as primary treatment option in the management of vestibular schwannomas (VS). In this "wait and scan" policy, audiovestibular symptoms are monitored regularly and VS growth is measured on consecutive magnetic resonance images (MRI). The aim of this study is validation of two-dimensional versus volume MRI assessment in the longitudinal follow-up of VS and to define tumor growth beyond measurement error.

\section{Materials and Methods}

MRI scans of 68 consecutive patients with VS were analyzed retrospectively. Two-dimensional and volume measurements on contrast enhanced (CE) T1-weighted and T2-weighted images were performed independently by two readers. Smallest detectable differences (SDD) were calculated and intra-class correlation coefficients (ICC) were determined for both assessment methods.

\section{Results}

Two-dimensional and volume measurements both showed best reproducibility on CE T1-weighted images. SDD for differences relative to baseline MRI [SDD(\%)] for two-dimensional measurements had a higher interobserver error compared to volume measurements ( $40 \%$ versus $19.7 \%)$, which decreases when tumor size increases. The ICC for two-dimensional measurements in three directions was $0.947,0.974$ and 0.978 and for volume measurements 0.999 .

\section{Conclusion}

Volume measurements are more accurate compared to two-dimensional measurements for the evaluation of VS growth. These measurements are assessed preferably on CE T1-weighted images. SDD (\%) strongly depends on VS size. SDD between consecutive scans exceeds the common clinical applied criterion of 1 or $2 \mathrm{~mm}$ growth to define growth. 


\section{Introduction}

The wide availability and technical improvement of magnetic resonance imaging (MRI) has led to an increase in detection of vestibular schwannoma (VS) at an early stage ${ }^{1}$. Accordingly, the incidence of diagnosed VS has increased $^{2,3}$. The natural development of VS remains uncertain as growth percentages between $30-90 \%$ have been reported, depending at least in part on the length of the observation period ${ }^{2,4}$. So far, no clinical parameters have been identified to correlate with VS growth ${ }^{5-7}$ and therefore VS growth is objectified by performing consecutive $\mathrm{MRI}^{2,5}$. If growth is found on MRI, an intervention may be chosen, such as surgical resection or radiation therapy ${ }^{6,8}$. Most patients therefore enter the so called "wait and scan" policy for a certain period, in which the audiovestibular symptoms are monitored regularly and VS growth is measured on consecutive $\mathrm{MRI}^{2}$.

Radiologists generally use two-dimensional measurements to assess VS growth, although volume measurements seem to provide more accurate growth assessment ${ }^{1,9,10}$ since VS shows asymmetric growth in all directions. Little information is published about the diameter increase or volume increment between subsequent images that constitute to VS growth beyond measurement error ${ }^{9-12}$. Usually, when an increase in tumor diameter or volume is found it is considered to be growing, but validation of this observation is lacking. Especially when invasive treatment decisions are based on these observations it is of great importance to find the most suitable method to assess growth of VS on MRI and to provide a definition of growth beyond measurement error.

This study focuses on the accuracy and reproducibility of VS volume measurements compared to two-dimensional measurements to determine VS growth on MRI. The hypothesis is that measurement of tumor volume with specific area tracing software is a more accurate tool compared to twodimensional measurements for determining tumor growth.

\section{Patients and methods}

All patients who received an MRI scan of the cerebellopontine angle (CPA) between January 2003 and March 2008 in our tertiary referral center were analyzed retrospectively. Patients were included in this study if a radiological diagnosis of a VS was made, resulting in 102 patients. Thirty patients were excluded who had been treated by surgery or radiotherapy, resulting in 72 patients. Four patients who had an intralabyrinthine schwannoma were also excluded. There were no patients with neurofibromatosis type 2 . MRI images of 68 patients, $32(47 \%)$ males and $36(53 \%)$ females, age range $36-84$ years, median age 63.5 years, were available; one scan was available in 21 patients, 
two scans were available in 22 patients, three scans in 10 patients, four scans in 8 patients, five scans in 4 patients and six scans in 3 patients, resulting in a total of 165 scans suitable for analysis. In patients with $>1$ scan, mean follow up was 21.8 months (SD 15.7).

All examinations were performed at $1.5 \mathrm{~T}$ (Gyroscan, Powertrack 6000, Philips, Best, The Netherlands) using a Head-Neck coil (Philips, Best, The Netherlands). The MR protocol consisted of axial 2D SE T1-weighted images (TR/TE, 550/15 ms; slice thickness, $3 \mathrm{~mm}$; inter slice gap, $0.3 \mathrm{~mm}$; number of slices, 12; FOV, $180 \mathrm{~mm}$ (RFOV 80\%); and matrix 256x256), axial 3D TSE T2-weighted images (TR/TE, 3,000/250 ms; slice thickness, $0.35 \mathrm{~mm}$; number of overcontiguous slices, 30; FOV, $130 \mathrm{~mm}$ (RFOV 80\%), and matrix 256x256) covering the skull base, and contrast enhanced (gadolinium $0.2 \mathrm{ml} / \mathrm{kg}$ body weight) axial 3D ISO T1-weighted images (TR/TE, 8.9/4.6 ms; slice thickness, $1 \mathrm{~mm}$; FOV, 256 (RFOV 80\%); and matrix 256x256) covering the entire skull base and cranium. All patients underwent the same MRI protocol with similar parameters and planes of acquisition to ascertain an optimal correlation in serial scans.

Two readers, experienced in head and neck imaging, independently performed the measurements on contrast enhanced T1-weighted images (CE T1-WI) and on the corresponding T2-weighted images (T2-WI). Both observers were blinded to each other's MR assessments and clinical information.

For two-dimensional assessment of VS, the maximum diameter was measured in three diameters: anteroposterior (AP), mediolateral (ML) [including the portion in the internal auditory canal, (IAC)], and craniocaudal (CC) (Figure 2.1A and 2.1B). To establish these dimensions, a digital submillimeter ruler was used. Volume assessment was done on a stereotactic radiotherapy treatment planning station, fitted with iPlan ${ }^{\circledR}$ RT image version 3-Advanced Contouring Workstation (BrainLAB Oncology Solutions, Feldkirchen, Germany). MR images were uploaded in this system, and area tracing software was used to outline the VS on each MR image that contained tumor tissue (Figure 2.2A). If there was a sharp contrast with surrounding tissue, the auto brush function (surrounding the VS automatically) was used. Manual segmentation was necessary in cases in which differentiation with surrounding tissue was difficult, because of the high sensitivity of this autotracer. Each segmentation result was checked visually. By tracing the VS surface on all slices the software was able to calculate VS volume (Figure 2.2B). Volumetric analysis was expressed in cubic centimeter.

To compare reproducibility of the measurements in different size categories, VS were classified into four stages, as defined by Hasegawa et al. ${ }^{13}$ : stage A, intracanalicular VS; stage $B$, VS extending into the CPA; stage C, VS compressing the brain stem and stage $D$, VS deviating the fourth ventricle. 

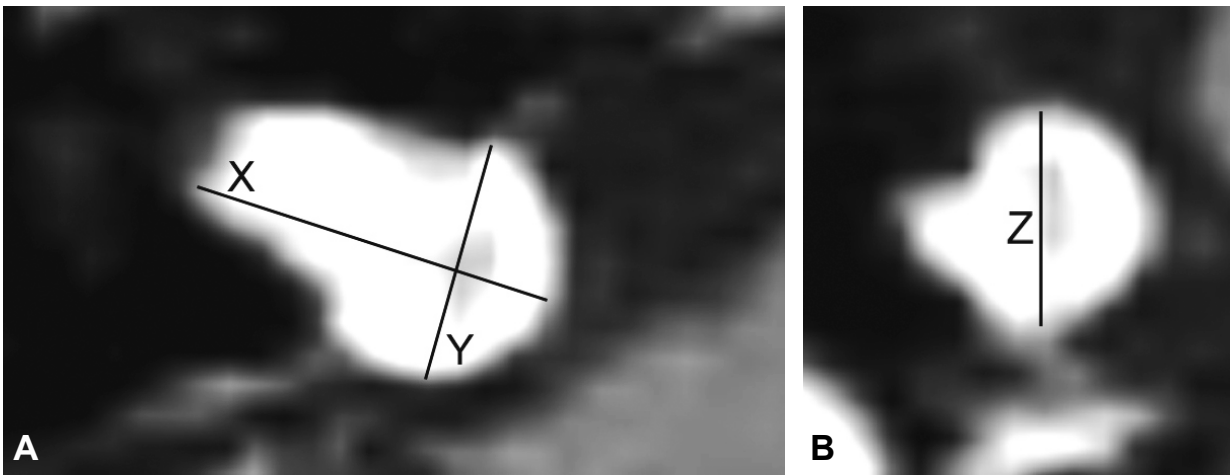

Figure 2.1 Contrast enhanced T1-weighted image with a vestibular schwannoma in the cerebellopontine angle on the right side. A. Measurements in the axial plane: $\mathrm{X}$ is the maximum mediolateral and $Y$ the maximum anteroposterior dimension. $B$. In the coronal plane the $Z$ demonstrates the maximum craniocaudal dimension.
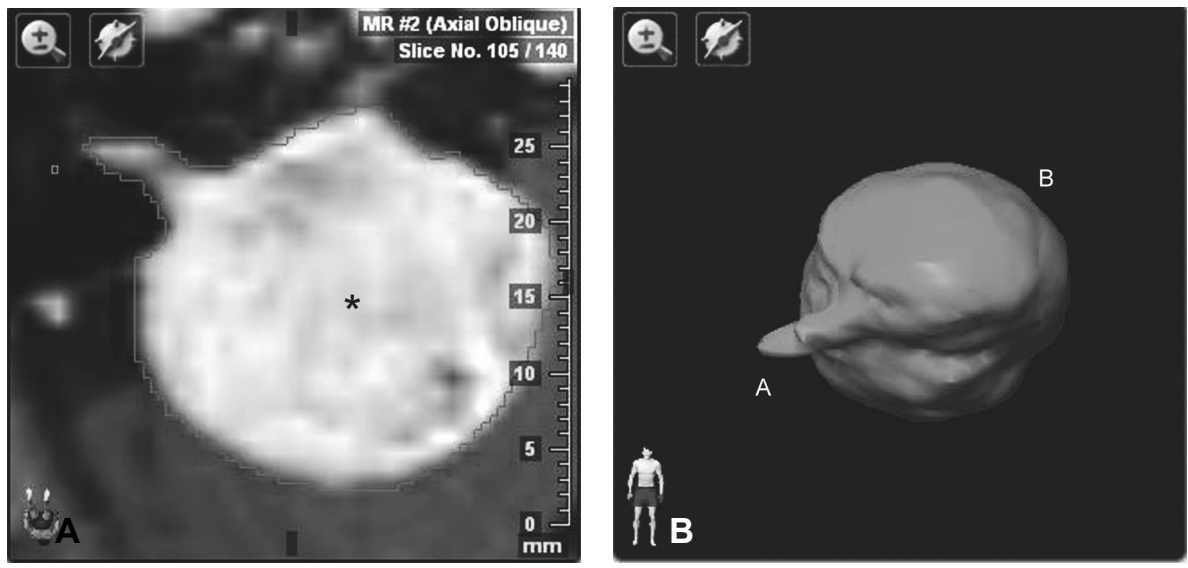

Figure 2.2 A. Example of area tracing with volume software. Axial contrast enhanced T1-weighted image shows a right sided vestibular schwannoma (asterix) with a large cerebellopontine angle component. The line around the VS is the result of the autotracer which lines the vestibular schwannoma. B. Three dimensional representation of a vestibular schwannoma (VS), integrating the surface of all slice intervals. The small intracanalicular (A) and large extracanalicular (B) portion of the VS can easily be identified.

\section{Statistical analysis}

Reproducibility measures consist of agreement and reliability parameters ${ }^{14}$. These parameters were evaluated for measurements by two different readers at one point in time. SPSS 15.0 statistical software (SPSS, Chicago, IL, USA) 
was used to perform the statistical calculations. For evaluation of interobserver reproducibility, we used baseline measurements of the MRI scans from the 68 patients for both readers, which can be considered as independent observations, whereas consecutive measurements within patients are correlated.

\section{Agreement parameters}

Agreement parameters measure the ability to achieve the same value in two measurements and give an indication of the size of measurement errors ${ }^{14}$. The agreement between two readers was evaluated using Bland and Altman plots $^{15}$. The Bland and Altman plot is the most robust method to quantify agreement in clinical measurements. Here, the differences between measurements (on the $Y$-axis) are plotted against the mean of two measurements (on the $X$-axis). The visual representation of agreement illustrates the magnitude and range of the differences, bias or outliers, and the relation between the magnitude of the differences and the magnitude of the mean values ${ }^{15}$. This method also assesses $95 \%$ limits of agreement. These limits of agreement are used to define the smallest detectable difference (SDD) as $1.96^{*}$ the standard deviation (SD) of the mean difference in measurements between the two readers. SDD represents the change that can be detected beyond measurement error ${ }^{15}$. We can define a tumor to have grown when the difference between two measurements falls outside this interval. In this way, it will be possible to discriminate between stable tumors and growing tumors, according to our measurements. For both two-dimensional and volume measurements, the smallest detectable difference for absolute (SDD) differences and for differences relative to baseline (SDD (\%)) was calculated. Differences relative to baseline were calculated using the following formula: $(A-B) /[(A+B) / 2]^{*} 100$, in which $A$ is the result of reader $A$ and $B$ the result of reader $B$. The SDD and SDD(\%) were presented for four VS stages: A, B, C and $\mathrm{D}$.

Use of the $\operatorname{SDD}(\%)$ enables comparison of the different unities involved in the two different measurement techniques (millimeter and cubic centimeter). Because in clinical practice all three diameter measurements (CC, AP and ML) are equally essential in assessing VS progression, we considered the diameter with lowest agreement as the limiting factor in diameter measurements. We compared the $\operatorname{SDD}(\%)$ of this diameter with the $\operatorname{SDD}(\%)$ of volume measurements.

\section{Reliability parameters}

Reliability parameters assess whether measurements can be used to distinguish patients from each other despite measurement error ${ }^{14}$. A parameter of reliability is the intraclass correlation coefficient (ICC). The ICCs are defined 
as the ratio of the variance among patients (patients variability) over the total variance (among patients, among readers, plus the error variance), which is expressed as a dimensionless number; being one (perfect reliability) in the most ideal case. ICC was calculated for interobserver diameter and volume VS measurement, and was also presented for four VS stages: A, B, C and D.

\section{Results}

Bland and Altman plots were constructed using data of the baseline MR images of the 165 scans from 68 patients (Figure 2.3 and 2.4). The standard deviation (SD) for each reader $(A$ and $B)$ and the $S D$ of the mean difference between readers are presented in Table 2.1. The SDD and SDD(\%) for absolute differences and differences relative to baseline MR images, respectively, are presented in Table 2.2. In Table 2.3 we present the ICC with $95 \%$ confidence intervals.

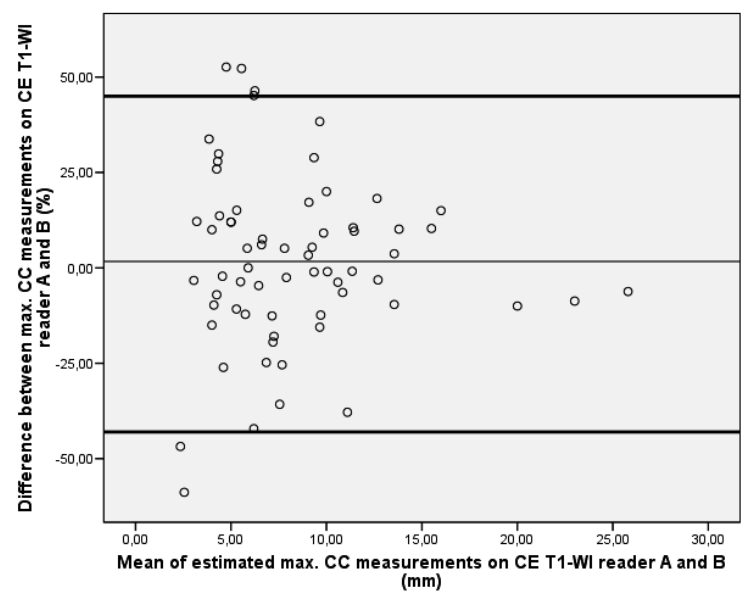

Figure 2.3 Bland and Altman plot of baseline two-dimensional maximum craniocaudal (CC) dimension measurements on contrast enhanced T1-weighted images. The values on the $Y$-axis represent the measurement differences between the two readers and their mean difference (thin line). The values on the $X$-axis represent the mean of both measurements. The thick black lines represent the $95 \%$ limits of agreement. Interobserver differences are larger in smaller vestibular schwannomas.

\section{Contrast enhanced T1- versus T2-weighted images}

Two-dimensional and volume assessments of VS were performed on both CE T1-WI (CC dimension, T1CC; AP dimension, T1AP; ML dimension, T1ML; volume, T1VOL) and T2-WI (CC dimension, T2CC; AP dimension, T2AP; ML 
dimension, T2ML; volume, T2VOL). The dimension with highest $\mathrm{SDD}(\%)$ was taken as a limiting factor, when comparing both imaging modalities.

With two-dimensional measurements, the $\operatorname{SDD}(\%)$ for T1CC appeared to be equal to T2CC (40.3 and 40.1) However, the T2AP dimension showed higher SDD(\%) compared to T1AP: 34.3 versus 28.3 , respectively. Therefore, CE T1-WI showed highest agreement in two-dimensional measurements. Also the ICC was consistently higher in T1CC, T1AP and T1ML directions, compared to T2CC, T2AP and T2ML directions $(0.947,0.974,0.978$ versus $0.943,0.961$, 0.948), reflecting higher reliability for CE T1-WI in two-dimensional measurements. For volume measurements, similar results were obtained: $\mathrm{SDD}(\%)$ for T1VOL was 19.7 compared to 30.1 in T2VOL. ICC values for volume measurements for both sequences were 0.999 .

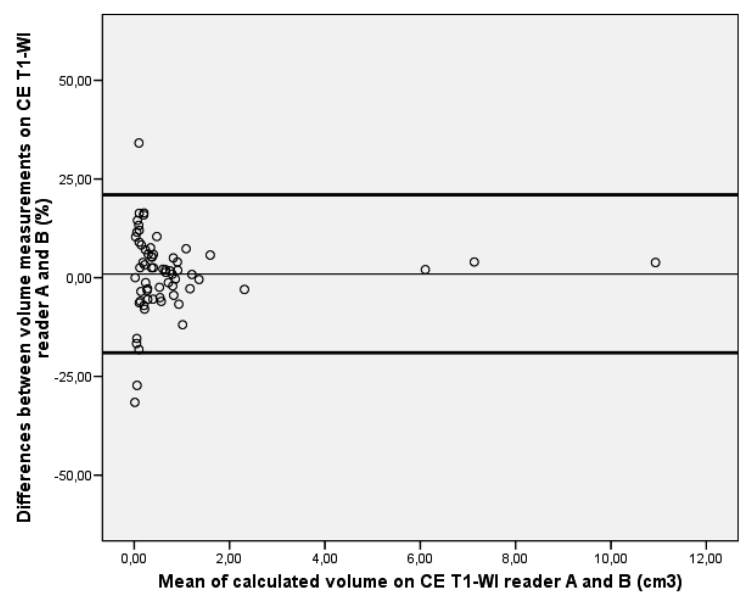

Figure 2.4 Bland and Altman plot of baseline volume measurements on contrast enhanced T1-weighted images. The values on the $Y$-axis represent the measurement differences between the two readers and their mean difference (thin line). The values on the $X$-axis represent the mean of both measurements. The thick black lines represent the $95 \%$ limits of agreement. Interobserver differences are larger in smaller vestibular schwannomas.

\section{Volume measurements versus two-dimensional measurements}

The SDD(\%) values for T1CC, T1AP and T1ML were 40.3, 28.3 and 20.9, respectively (Table 2.2). All three dimensions are equally essential in estimating VS growth. Because the T1CC dimension is the limiting factor in these two-dimensional measurements, with its lowest agreement, it was used to compare the two-dimensional measurements with volume measurements. The $\operatorname{SDD}(\%)$ for T1CC was considerably higher than the $\operatorname{SDD}(\%)$ for volume measurements (T1VOL): $40.3 \%$ versus $19.7 \%$. The $\operatorname{SDD}(\%)$ decreased with 
increasing tumour size from stage $A$ to stage $D$ VS, in both two-dimensional and in volume measurements (Table 2.2). In all tumor stages, volume measurements were associated with smaller $\operatorname{SDD}(\%)$ compared to $\mathrm{CC}$ dimensional measurements. The ICC revealed that both two-dimensional and volume measurements showed high interobserver reliability. However, volume measurements revealed a higher reliability compared to the three diameter measurements (0.999 versus $0.947,0.974$ and 0.978 ). Reliability increased with tumor size and volume measurements were more reliable in all VS stages (Table 2.3).

Table 2.1 Interobserver agreement parameters based on baseline contrast enhanced T1-weighted, images (CE T1-WI) and T2-weighted images in 68 patients.

\begin{tabular}{|c|c|c|c|c|}
\hline \multirow[t]{2}{*}{ Baseline } & \multicolumn{3}{|c|}{ Two-dimensional measurements on CE T1-WI (mm) } & \multirow{2}{*}{$\begin{array}{c}\text { Volume measurements } \\
\text { on CE T1-WI }\left(\mathrm{cm}^{3}\right) \\
\text { Vol }\end{array}$} \\
\hline & $\mathrm{CC}$ & AP & $M L$ & \\
\hline Obs A : mean (SD) & $8.40(4.60)$ & $8.70(4.90)$ & $12.70(5.70)$ & $0.85(1.74)$ \\
\hline A & 5.00 & 5.06 & 8.32 & 0.13 \\
\hline B & 9.05 & 9.71 & 14.28 & 0.63 \\
\hline C & 12.58 & 12.23 & 18.12 & 1.29 \\
\hline D & 17.50 & 18.33 & 21.62 & 4.71 \\
\hline Obs B : mean (SD) & $8.20(4.80)$ & $8.60(5.10)$ & $13.20(5.70)$ & $0.81(1.69)$ \\
\hline A & 4.59 & 5.03 & 8.58 & 0.13 \\
\hline B & 9.27 & 9.86 & 15.06 & 0.63 \\
\hline C & 11.53 & 13.10 & 18.28 & 1.06 \\
\hline D & 18.33 & 18.93 & 22.28 & 4.57 \\
\hline $\operatorname{Mean}_{\text {diff }}\left(S D_{\text {diff }}\right)$ & $0.12(1.53)$ & $-0.18(1.14)$ & $-0.48(1.08)$ & $0.01(0.055)$ \\
\hline \multirow[t]{2}{*}{ Baseline } & \multicolumn{3}{|c|}{ Diameter measurements on T2 -WI (mm) } & Volume measurements \\
\hline & $\mathrm{CC}$ & AP & $\mathrm{ML}$ & Vol \\
\hline Obs A : mean (SD) & $7.60(4.5)$ & $8.20(5.00)$ & $11.90(5.50)$ & $0.72(1.49)$ \\
\hline Obs B : mean (SD) & $7.20(4.00)$ & $8.40(4.90)$ & $12.10(5.00)$ & $0.72(1.50)$ \\
\hline$M_{\text {ean }}$ diff $\left(S D_{\text {diff }}\right)$ & $0.35(1.41)$ & $-0.18(1.37)$ & $-0.21(1.69)$ & $0.07(0.069)$ \\
\hline
\end{tabular}

The rows of reader $A$ and $B$ of each imaging modality present the means and standard deviations of the measurements assessed by these readers. The means and standard deviations are also subdivided according to vestibular schwannoma stage for CE T1-WI. The mean difference row presents the mean differences between the measurements of reader $A$ and $B$ and the SD of these differences. A: intracanalicular; B: extracanalicular; C: compressing brain stem; D: deviating the fourth ventricle. $\mathrm{CC}=$ craniocaudal dimension, $\mathrm{AP}=$ anteroposterior dimension, $\mathrm{ML}=$ mediolateral dimension, $\mathrm{VOL}=$ volume, Mean diff : mean of the observer difference. (SD diff): standard deviation of the differences between observer A and B, CE T1-WI: contrast enhanced T1 weighted images, T2WI: T2 weighted images. 


\section{Smallest detectable differences in two-dimensional and volume measurements}

SDD for two-dimensional measurements on CE T1-WI varied from 2.12-2.98 mm. For volume measurements on CE T1-WI SDD was $0.11 \mathrm{~cm} 3$ (Table 2.2).

Table 2.2 SDD and SDD(\%) for measurements performed on contrast enhanced T1-weighted images and T2-weighted images.

$\begin{array}{llllllllll} & \text { CC } & \text { CC } & \text { AP } & \text { AP } & \text { ML } & \text { ML } & \text { Vol } & \text { Vol } \\ & \text { SDD } & \text { SDD(\%) } & \text { SDD } & \text { SDD(\%) } & \text { SDD } & \text { SDD(\%) } & \text { SDD } & \text { SDD(\%) } \\ \text { T1 A-D } & 2.98 & 40.3 & 2.23 & 28.3 & 2.12 & 20.9 & 0.11 & 19.7 \\ \text { T2 A-D } & 2.76 & 40.1 & 2.69 & 34.3 & 2.69 & 30.52 & 0.14 & 30.1 \\ \text { A }(n=29) & 2.41 & 52.8 & 1.49 & 28.0 & 1.96 & 27.1 & 0.05 & 28.9 \\ \text { B }(n=27) & 3.23 & 34.5 & 2.53 & 26.5 & 2.33 & 17.8 & 0.06 & 10.4 \\ \text { C }(n=6) & 3.60 & 32.7 & 3.05 & 28.7 & 2.49 & 14.9 & 0.07 & 8.7 \\ \text { D }(n=6) & 2.96 & 18.8 & 2.90 & 23.7 & 1.08 & 5.0 & 0.26 & 5.7\end{array}$

The SDD and SDD(\%) for CE T1-WI are subdivided according to vestibular schwannoma (VS) stage: $A=$ intracanalicular $V S, B=V S$ extending into the cerebellopontine angle, $C=V S$ compressing brain stem, $D=V S$ deviating the fourth ventricle. $S D D=$ smallest detectable difference, $S D D(\%)=$ smallest detectable difference relative to baseline measurements, $\mathrm{CC}=$ craniocaudal dimension, $\mathrm{AP}=$ anteroposterior dimension, $\mathrm{ML}=$ mediolateral dimension, $\mathrm{VOL}=$ volume.

Table 2.3 Intraclass correlation coefficients (ICC) for two-dimensional and volume measurements on contrast enhanced T1-weighted images and T2-weighted images

$\begin{array}{lcccc} & \mathrm{CC} & \mathrm{AP} & \mathrm{ML} & \mathrm{VOL} \\ \text { T1 A-D } & 0.947(0.915-0.967) & 0.974(0.957-0.984) & 0.978(0.965-0.987) & 0.999(0.999-1.000) \\ \text { T2 A-D } & 0.943(0.909-0.965) & 0.961(0.938-0.976) & 0.948(0.917-0.968) & 0.999(0.998-0.999) \\ \mathrm{A}(\mathrm{n}=29) & 0.564(0.260-0.768) & 0.862(0.729-0.932) & 0.947(0.892-0.975) & 0.987(0.973-0.994) \\ \mathrm{B}(\mathrm{n}=27) & 0.674(0.393-0.841) & 0.802(0.604-0.907) & 0.889(0.767-0.949) & 0.988(0.973-0.994) \\ \mathrm{C}(\mathrm{n}=6) & 0.840(0.315-0.975) & 0.917(0.588-0.988) & 0.968(0.822-0.995) & 0.999(0.993-1.000) \\ \mathrm{D}(\mathrm{n}=6) & 0.966(0.810-0.995) & 0.980(0.883-0.997) & 0.990(0.942-0.999) & 0.999(0.992-1.000)\end{array}$

ICC values for CE T1-WI are subdivided per VS stage: $A=$ intracanalicular VS, $B=V S$ extending into the cerebellopontine angle, $\mathrm{C}=\mathrm{VS}$ compressing brain stem, $\mathrm{D}=\mathrm{VS}$ deviating the fourth ventricle. $\mathrm{CC}=$ craniocaudal dimension, $\mathrm{AP}=$ anteroposterior dimension, $\mathrm{ML}=$ mediolateral dimension, $\mathrm{VOL}=$ volume.

\section{Discussion}

VS are benign neoplasms, originating from the neurolemnal sheath of the eighth cranial nerve and are predominantly found in the CPA and in the IAC. The incidence of VS varies from 1-2/100.000 2,12,16,17, although postmortem histopathological examinations show a higher incidence of about $2.7 \%{ }^{18}$. This discrepancy indicates that the vast majority of VS never become symptomatic, reflecting very slow or arrested growth. 
Therefore, the wait and scan policy has gained popularity as an alternative or prelude to surgery and radiation therapy. This can be justified as growth is known to be extremely variable with most VS remaining stable or showing minimal growth for many years ${ }^{16,19}$. The goal of this regimen is to minimize therapeutic risks and complications and to preserve an optimal quality of life in selected patients. Because no single reliable clinical feature exists that predicts tumor growth ${ }^{5-7}, \mathrm{MRI}$ is the mainstay in the conservative management of $\mathrm{VS}^{2,5}$. It is essential to use a measuring method that provides reliable measurements with a high interobserver agreement, as change in size is - besides its clinical presentation - the most relevant parameter.

Various ways of describing VS tumor growth have been proposed. The conventional method of assessing VS is by performing two-dimensional measurements. Some authors use the largest AP and/or ML dimension, sometimes combined with the $\mathrm{CC}$ dimension ${ }^{5,8,19-24}$. Others use the guidelines for measuring VS described by the American Academy of Otolaryngology, Head and Neck Surgery (AAO-HNS) ${ }^{6,7,25}$. The usefulness of two-dimensional measurements could be doubted. Firstly, two-dimensional measurements assesses VS growth in maximal three directions, while a VS shows asymmetric growth in all possible directions; therefore a two-dimensional assessment does not approach "real" tumor growth. Secondly, in VS with large diameters, a small increase in diameter corresponds to a much larger increase in VS volume than a similar increase in diameter in a small VS ${ }^{1}$. Volume measurements can be performed in several ways; some authors consider a VS to be an ellipsoid and calculate the volume using a mathematical formula $a^{11,21,26,27}$. However, this method has shown to produce a large overestimation of VS volume ${ }^{1,11}$. Others have performed true volumetric measurements by using (semi)automatic software to calculate VS volume ${ }^{1,9,11,12,21,28,29}$. According to the few studies comparing two-dimensional versus non-formula-based volume measurements, the VS volume measurements are more accurate compared to two-dimensional measurements ${ }^{1,9,10}$. Other authors disagree with this ${ }^{16,21,27}$ and also in clinical practice, most clinicians keep relying on two-dimensional measurements. However, the results of this study indicate that VS volume measurements, especially on the CE T1-WI, produce a better interobserver agreement and reliability compared to the two-dimensional measurements. This study therefore indicates that CE T1-WI volume measurements should replace twodimensional measurements in evaluating VS growth. The difference in interobserver agreement and reliability between the two measurement methods is of clinical significance, because invasive treatment decisions are based on these observations. Therefore the measurement method with highest agreement en reliability is necessary in assessing VS growth.

An exception may be made concerning small (stage A) VS. In these small intracanalicular VS, the CC dimension does not play an important role since the 
diameter of the internal auditory canal is usually quite constant. When one uses the AAO-HNS guidelines, the CC direction is not taken into account at all. Then, the AP direction is the limiting factor of the remaining $A P$ and $M L$ directions (Table 2.2). In stage A VS, under these conditions, we found that measurement error [SDD(\%)] of both two-dimensional and volume measurement techniques are comparable (AP 28.0, ML 27.1, VOL 28.9). Therefore, both measurement techniques can be used to evaluate stage A VS. In larger VS (stage $B$ and $C$ ), both $A P$ and $M L$ dimension show higher measurement error $[\mathrm{SDD}(\%)]$ compared to volume measurements. In stage $\mathrm{D}$ VS, the $\operatorname{SDD}(\%)$ of the ML dimension equals the $\operatorname{SDD}(\%)$ of volume measurements: 5 versus 5.7 respectively. This can be explained by the fact that the ML dimension is the longest distance in two dimensional VS measurements and measurement error will decrease when measurement distances increase. This occurs both in two-dimensional and in volume measurements and should be taken into account when evaluating growth in different VS stages (Table 2.2, Figure 2.3 and 2.4). Other authors confirm this phenomenon ${ }^{1,11,30}$. However, apart from this low $\operatorname{SDD}(\%)$ of the $\mathrm{ML}$ dimension in stage D VS, the far worse $\operatorname{SDD}(\%)$ of the AP dimension should also be taken into account in evaluating VS growth with two-dimensional measurements, thus pleading for the use of volume measurements in the assessment of stage B, C and D VS.

Overall, contrast enhanced images are necessary, both in volume and twodimensional measurements, to maintain a high reproducibility, since it facilitates differentiation of VS from surrounding tissue.

The calculated agreement [SDD and $\operatorname{SDD}(\%)$ ] and reliability (ICC) values were compared with findings reported in the literature. Studies addressing the change in length and volume exceeding measurement error (SDD) are reported inconclusively. In two-dimensional VS measurements many authors simply state a 1 or $2 \mathrm{~mm}$ increase in subsequent scans as evidence of growth without any statistical justification ${ }^{2,6-8,20,22,31}$. According to this present study, SDD varies between $2.12 \mathrm{~mm}$ for the $\mathrm{ML}$ dimension and $2.98 \mathrm{~mm}$ for the CC dimension on CE T1-WI (Table 2.2). This indicates that there is a measurement error in two-dimensional measurements which is not recognized when a measured increase in size of only 1 or $2 \mathrm{~mm}$ between follow-up scans is considered as tumor growth, and treatment decisions should be used with caution when using these arbitrary criteria.

In volume measurements, an absolute increase above which one can consider a VS to have grown, was not found in the literature. This study revealed SDD ranging from $0.05-0.26 \mathrm{~cm}^{3}$ (type A-D) on CE T1-WI (Table 2.2). Volume increase expressed as SDD(\%) varied from $15-89 \%$ in the literature ${ }^{9-12}$. However, the numbers of patients in these studies were small ${ }^{9,11,12}$ and generally, not only baseline measurements of the VS were taken into 
account $^{9,10,12}$. Therefore, the percentages reported in the literature could be questioned. In this study, $\operatorname{SDD}(\%)$ in CE T1-WI volume measurements was $19.7 \%$. ICC for two-dimensional measurements was not found in the literature. One study calculated ICC for volume measurements: Luppino et al. ${ }^{9}$ calculated ICC for two different types of volume measurement. Their "contour method", similar to our volume method, had an interobserver reliability of 0.96 , which is comparable to the ICC of 0.99 in this study.

This study and technique also harbor some limitations. Firstly, the assessments of reproducibility parameters were based on interobserver differences and not on intraobserver differences. It was assumed that this approach better reflects clinical practice, where it is usual that different clinicians assess subsequent scans.

Secondly, Brain $\angle A B$ volume software is not widely available in radiology departments.

Thirdly, performing these volume measurements takes a little more time compared to the conventional two-dimensional measurements. In our experience, VS contouring and volume calculation typically took a few minutes. This could be a limiting factor in introducing this method for VS in daily clinical practice. In the literature, 10-25 minutes for manual segmentation has been described, although these calculations were performed with different software and on older systems ${ }^{10,11}$.

Fourthly, the used volume measurement method is semi-automatic. However, there is still a human component, responsible for an interobserver difference. It is desirable to develop software able to perform even better automated volume measurements, in order to further diminish reader related measurement error.

\section{Conclusion}

CE T1-WI volume measurements show better interobserver agreement and reliability compared to two-dimensional measurements for the assessment of growth of VS. Small intracanalicular VS form an exception. When evaluating VS growth, one has to take VS baseline characteristics into account, because $\operatorname{SDD}(\%)$ strongly depends on VS size. The 1 or $2 \mathrm{~mm}$ difference commonly used to define growth of VS in consecutive scans in two-dimensional measurements lies within measurement error and should not direct clinical practice. 


\section{References}

1. Shin YJ, Fraysse B, Cognard C, Gafsi I, Charlet JP, Berges C, Deguine O, Tremoulet M. Effectiveness of conservative management of acoustic neuromas. Am J Otol. 2000;21:857-62.

2. Stangerup SE, Caye-Thomasen $P$, Tos $M$, Thomsen J. The natural history of vestibular schwannoma. Otol Neurotol. 2006;27:547-52.

3. Stangerup SE, Caye-Thomasen $\mathrm{P}$, Tos M, Thomsen J. Change in hearing during 'wait and scan' management of patients with vestibular schwannoma. J Laryngol Otol. 2008;122:673-81.

4. Charabi S, Tos M, Thomsen J, Charabi B, Mantoni M. Vestibular schwannoma growth: the continuing controversy. Laryngoscope. 2000;110:1720-5.

5. Slattery WH, 3rd, Fisher LM, Yoon G, Sorensen G, Lev M. Magnetic resonance imaging scanner reliability for measuring changes in vestibular schwannoma size. Otol Neurotol. 2003;24:666-70.

6. Walsh RM, Bath AP, Bance ML, Keller A, Tator $\mathrm{CH}$, Rutka JA. The role of conservative management of vestibular schwannomas. Clin Otolaryngol Allied Sci. 2000;25:28-39.

7. Raut VV, Walsh RM, Bath AP, Bance ML, Guha A, Tator CH, Rutka JA. Conservative management of vestibular schwannomas - second review of a prospective longitudinal study. Clin Otolaryngol Allied Sci. 2004;29:505-14.

8. Smouha EE, Yoo M, Mohr K, Davis RP. Conservative management of acoustic neuroma: a meta-analysis and proposed treatment algorithm. Laryngoscope. 2005;115:450-4.

9. Luppino FS, Grooters E, de Bruine FT, Zwinderman AH, van der Mey AG. Volumetrical measurements in vestibular schwannoma, the influence of slice thickness and patient's repositioning. Otol Neurotol. 2006;27:962-8.

10. Vokurka EA, Herwadkar A, Thacker NA, Ramsden RT, Jackson A. Using Bayesian tissue classification to improve the accuracy of vestibular schwannoma volume and growth measurement. AJNR Am J Neuroradiol. 2002;23:459-67.

11. Cross JJ, Baguley DM, Antoun NM, Moffat DA, Prevost AT. Reproducibility of volume measurements of vestibular schwannomas - a preliminary study. Clin Otolaryngol. 2006;31:123-9.

12. Niemczyk K, Vaneecloo FM, Lemaitre L, Lejeune JP, Skarzynski H, Dubrulle F, Vincent $C$. The growth of acoustic neuromas in volumetric radiologic assessment. Am J Otol. 1999;20:244-8.

13. Hasegawa T, Fujitani S, Katsumata S, Kida Y, Yoshimoto M, Koike J. Stereotactic radiosurgery for vestibular schwannomas: analysis of 317 patients followed more than 5 years. Neurosurgery. 2005;57:257-65.

14. de Vet HC, Terwee CB, Knol DL, Bouter LM. When to use agreement versus reliability measures. J Clin Epidemiol. 2006;59:1033-9.

15. Bland JM, Altman DG. Statistical methods for assessing agreement between two methods of clinical measurement. Lancet. 1986;1:307-10.

16. Rosenberg SI. Natural history of acoustic neuromas. Laryngoscope. 2000;110: 497-508.

17. Tos M, Charabi S, Thomsen J. Clinical experience with vestibular schwannomas: epidemiology, symptomatology, diagnosis, and surgical results. Eur Arch Otorhinolaryngol. 1998;255:1-6.

18. Yoshimoto $\mathrm{Y}$. Systematic review of the natural history of vestibular schwannoma. $J$ Neurosurg. 2005;103:59-63. 
19. Selesnick $\mathrm{SH}$, Johnson $\mathrm{G}$. Radiologic surveillance of acoustic neuromas. Am J Otol. 1998;19:846-9.

20. Doherty JK, Friedman RA. Controversies in building a management algorithm for vestibular schwannomas. Curr Opin Otolaryngol Head Neck Surg. 2006;14:305-13.

21. Fiirgaard B, Pedersen CB, Lundorf E. The size of acoustic neuromas: CT and MRI. Neuroradiology. 1997;39:599-601.

22. Fucci MJ, Buchman CA, Brackmann DE, Berliner KI. Acoustic tumor growth: implications for treatment choices. Am J Otol. 1999;20:495-9.

23. Tanaka $\mathrm{Y}$, Hongo K, Tada $\mathrm{T}$, Kobayashi $\mathrm{S}$. What is the best method for reporting tumor diameter in vestibular schwannoma? Neurosurgery. 2003;53:634-7.

24. Saito Al, Morris CG, Ito K, Watanabe F, Karasawa K, Mendenhall WM, Naoi Y. Comparing size evaluation methods for acoustic neuroma after stereotactic radiosurgery. Radiat Med. 2007;25:339-45.

25. Committee on Hearing and Equilibrium guidelines for the evaluation of hearing preservation in acoustic neuroma (vestibular schwannoma). American Academy of Otolaryngology-Head and Neck Surgery Foundation, INC. Otolaryngol Head Neck Surg. 1995;113:179-80.

26. Caye-Thomasen P, Hansen S, Dethloff T, Stangerup SE, Thomsen J. Sublocalization and volumetric growth pattern of intracanalicular vestibular schwannomas. Laryngoscope. 2006;116:1131-5.

27. Charabi S, Thomsen J, Mantoni M, Charabi B, Jorgensen B, Borgesen SE, Gyldensted C, Tos M. Acoustic neuroma (vestibular schwannoma): growth and surgical and nonsurgical consequences of the wait-and-see policy. Otolaryngol Head Neck Surg. 1995;113:5-14.

28. Laasonen EM, Troupp $H$. Volume growth rate of acoustic neurinomas. Neuroradiology. 1986;28:203-7.

29. Meijer OW, Weijmans EJ, Knol DL, Slotman BJ, Barkhof F, Vandertop WP, Castelijns JA. Tumor-volume changes after radiosurgery for vestibular schwannoma: implications for follow-up MR imaging protocol. AJNR Am J Neuroradiol. 2008;29:906-10.

30. Sarazin L, Jolivet O, Doyon D. Computerized evaluation of tumor volume with MRI. Applications to the surveillance of acoustic neurinoma. J Radiol. 1993;74:455-60.

31. Bozorg Grayeli A, Kalamarides M, Ferrary E, Bouccara D, El Gharem H, Rey A, Sterkers O. Conservative management versus surgery for small vestibular schwannomas. Acta Otolaryngol. 2005;125:1063-8. 



\section{PREDICTORS OF VOLUMETRIC GROWTH AND AUDITORY DETERIORATION IN VESTIBULAR SCHWANNOMAS FOLLOWED IN A WAIT AND SCAN POLICY}

Rick van de Langenberg Bert J de Bondt Patty J Nelemans Amy JC Dohmen Brigitta G Baumert

Robert J Stokroos

Otology \& Neurotology. 2011;32:338-344 


\section{Abstract}

\section{Objective}

The purpose of this study was to identify factors predicting growth and audiologic deterioration during follow-up (FU) in a wait and scan (W\&S) policy of vestibular schwannomas (VSs) using a novel volumetric measuring tool. So far, only consecutive magnetic resonance imaging (MRI) is able to show growth objectively, and growth, combined with hearing function, generally dictates further intervention. Other factors predicting growth or hearing deterioration would be invaluable and might ease clinical decision making.

\section{Study Design}

Retrospective case study

\section{Setting}

Tertiary referral center

\section{Patients}

Sixty-three patients diagnosed with VS at Maastricht University Medical Center between 2003 and 2008, with FU data available from 36 patients.

Intervention(s): A W\&S policy for unilateral VS with sequential contrast enhanced T1- and T2weighted MRI and audiograms during FU.

\section{Main Outcome Measure(s)}

1. Patient and radiologic VS features potentially related to growth and auditory function during a W\&S policy. 2. The correlation between increase in VS volume and audiologic deterioration during FU.

\section{Results}

Labyrinthine hypointensity on T2-weighted MRI and complaints of hearing loss at presentation are predictive of a faster deterioration of hearing $(P<0.05)$. Growth during the first FU year predicts further growth. VS volume does not correlate with audiologic deterioration significantly.

\section{Conclusions}

Hypointensity on T2-weighted MRI of the affected labyrinth will result in a significant faster deterioration of hearing. Hearing loss was more profound, and hearing will deteriorate significantly faster in patients presenting with complaints of hearing loss. Significant growth during the first year of FU predicts further growth during FU. Sequential MRI can not be substituted by audiologic examinations solely, because increase in VS volume does not correlate with audiologic deterioration significantly. 


\section{Introduction}

The vestibular schwannoma (VS) is a benign neoplasm originating from the $\mathrm{VIII}^{\text {th }}$ cranial nerve. Diagnosis may be occurring more frequently with widespread use of magnetic resonance imaging (MRI) in populations that are living longer. With increasing rates of diagnosis, clinical decision making may benefit from better tumor measurement and knowledge of growth characteristics. VS growth is known to be highly variable with annual growth numbers of $30 \%$ to $90 \%$, depending at least in part on the length of the observation period ${ }^{1-3}$. Microsurgery (MS) and stereotactic radiotherapy (SRT) are regarded as the main treatment options ${ }^{2,4-6}$. An increasing number of patients enter "wait and scan" (W\&S) policy, in which the audiovestibular function of the patient is evaluated and VS size is monitored by consecutive MRI. Progressive sensorineural hearing loss is the symptom reported most often at the time of VS diagnosis, and loss of hearing, as well as growth, may continue during this W\&S policy ${ }^{7-9}$. So far, sequential MRI is the only objective method to identify growth and is, combined with clinical symptoms such as hearing deterioration, generally decisive in clinical decision making ${ }^{10,11}$. This study focuses on determination of patient and radiologic VS-related factors predicting VS growth or hearing loss. Identification of these factors would be invaluable because they might ease clinical decision making and advising VS patients. For measuring VS size, a novel volumetric measuring tool was used, which is more sensitive compared with the 2-dimensional measurements ${ }^{12}$, used in daily practice and in previous studies s, $^{2,3-10,13-28}$.

\section{Materials and methods}

All 102 consecutive patients who received an MRI scan of the cerebellopontine angle (CPA) and who were diagnosed with a VS between January 2003 and July 2008 at Maastricht University Medical Center (a tertiary referral center), were evaluated retrospectively. Patients were eligible for the study if a radiologic diagnosis of a VS was made and patients were candidates for a W\&S policy. Excluded were 30 patients who were immediately treated by surgery or radiotherapy and 4 patients who had an intralabyrinthine schwannoma, resulting in a study population of 68 patients. In 5 patients an audiogram was unavailable leaving a study group of 63 patients with a unilateral VS with at least one combination of audiogram/MRI available for analysis.

A retrospective chart review was performed on these patients; data on sex and age at presentation were noted. Presence or absence of 3 major symptoms at presentation were recorded: presence of complaint of hearing loss, tinnitus and 
vertigo. These symptoms were reassessed annually, together with audiologic testing and a follow-up (FU) MRI.

Audiometric assessments were recorded in accordance with American Academy of Otolaryngology, Head and Neck Surgery (AAO-HNS) guidelines and consisted of the mean pure tone average (PTA, mean hearing threshold at $0.5,1,2$ and $4 \mathrm{kHz}$ ), speech discrimination score (SDS) and hearing class (A, $\mathrm{B}, \mathrm{C}$ and $\mathrm{D})^{29}$.

MRI sequences included T2-weighted images (T2-WI) and contrast enhanced T1-weighted images (CE T1-WI) in the axial plane. Slice thickness was $1 \mathrm{~mm}$; there was no slice gap. The volume measurements on axial CE T1-WI were performed by a reader, experienced in VS imaging, using a computer system fitted with specialized semi automatic software: iPlan® RT Image version 3 (BrainLab Oncology Solutions, Feldkirchen, Germany). Area tracing software was used to outline the VS on each MR image that contained tumor tissue. If there was a sharp contrast with surrounding tissue, the auto brush function (surrounding the VS automatically) was used. Manual segmentation was necessary in cases in which differentiation with surrounding tissue was difficult because of the high sensitivity of this autotracer. Each segmentation result was checked visually. By tracing the VS surface on all slices, the software was able to calculate VS volume. Volumetric analysis was expressed in cubic centimeter $\left(\mathrm{cm}^{3}\right)$. In our experience, VS contouring and volume calculation typically took one or two minutes. Morphological features were assessed as follows: signal intensity of the labyrinth was scored as either isointense compared with the contralateral labyrinth or hypointense; this observation was performed on T2-WI (Figure 3.1 and 3.2). As previously defined by Hasegawa et al. VS were staged in 4 subtypes according to their appearance on CE-T1WI: A: remaining in the internal auditory canal (IAC), B: extending in the CPA C: compressing the brain stem, D: deviating the fourth ventricle ${ }^{4}$. VS side was scored left or right. Further technical details regarding MRI, the parameters of the sequences used and measurement technique are published elsewhere ${ }^{12}$. Intervention was defined as either MS or SRT.

Significant growth was defined as a percentage change in VS volume between 2 subsequent scans that exceeded $19.7 \%$. In clinical practice, this value may more readily be rounded to $20 \%$ in order to make it more clinically applicable. In a previous study, this value was identified as the mean of the smallest detectable change (SDD\%) between subsequent scans, which is considered as definition of growth, detected beyond measurement error for volume measurements $(P<0.05)^{12}$. Volume measurements were performed in this study, because the SDD\% of $2 \mathrm{D}$ measurements in the commonly used mediolateral and anteroposterior VS axis were worse compared to volume measurements, for stage $B, C$ and $D$ and comparable only in stage $A$ VS. 


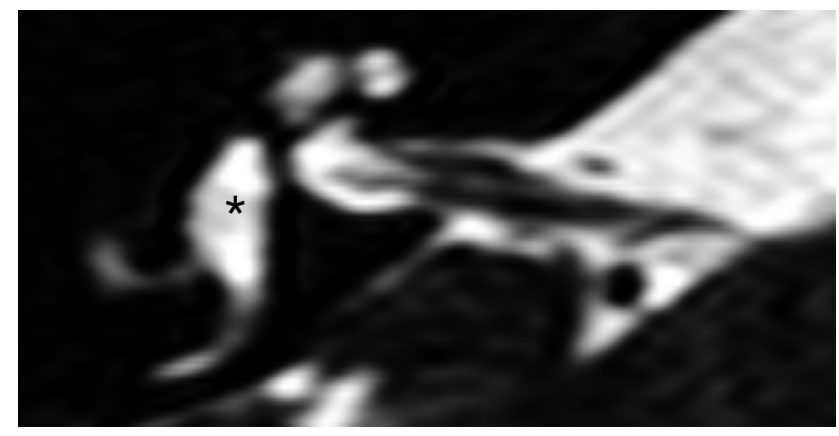

Figure 3.1 The right labyrinth (black asterisk) appearing with a isointense signal intensity on T2-weighted MRI.

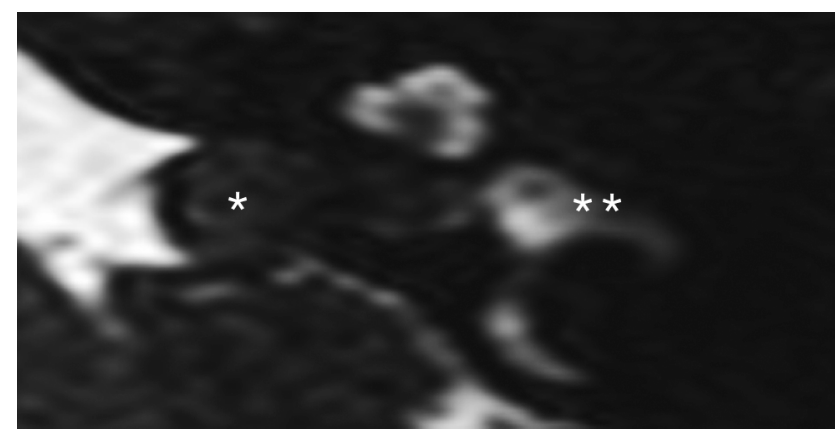

Figure 3.2 The left labyrinth (double white asterisk) appears hypointense, indicating a loss or change of inner ear fluids. A vestibular schwannoma (white asterisk) can be seen in the left internal auditory canal.

\section{Statistical analysis}

Absolute changes in volume were calculated by subtracting the VS volume at diagnosis from volume at the last measurement (volume at $t_{x}$-volume at $t_{0}$ ). Relative changes in volume were calculated as absolute change divided by the VS volume at diagnosis: (volume at $t_{x}$-volume at $t_{0}$ ) / volume at $t_{0}$. This relative change was multiplied by 100 to gain a percentage change in volume. Fisher exact and $\chi^{2}$ test were used for categorical data. Differences in mean values were tested for significance using the independent samples t-test for comparison between two groups and paired samples t-test for comparing means of two variables. Multivariate analyses were performed to estimate the independent effects of various baseline factors by mutual adjustment of variables incorporated in the models. Regression coefficients $(\beta)$ with corresponding $95 \%$ confidence intervals were calculated. Logistic analyses were performed with absence or presence of significant growth as dependent variable. In this case, odds ratios were derived by exponentiation of regression coefficients and represent the strength of the association between baseline 
variables and significant growth. Linear analyses were performed with change in PTA or change in SDS as dependent variable. In this case, the regression coefficient $(\beta)$ represents the mean difference in hearing loss between the groups with and without a specific baseline characteristic. The $95 \%$ confidence intervals indicate whether the regression coefficient differs significantly from zero. For all tests, P-values $\leq 0.05$ were considered to indicate statistical significance. All analyses were performed using SPSS 15.0 statistical software (SPSS, Chicago, IL, USA).

\section{Results}

\section{Study Group and Tumor Characteristics}

The study group consisted of 63 patients. In 27 patients, only one baseline MRI/audiogram measurement at the time of diagnosis was available. In 36 patients, FU MRI/audiogram measurements were available. Two measurements were available in 19 patients, 3 measurements in 9 patients, 4 measurements in 4 patients, 5 measurements in 3 patients, and 6 measurements in 1 patient, resulting in a total of 129 measurements suitable for analysis. The group with FU measurements consisted of 17 men, 19 women, median age 58.5 years (range $41-78 \mathrm{yr}$ ). Thirty-one $(86 \%)$ of 36 complained of hearing loss, $12(33 \%)$ complained of tinnitus and 10 patients $(30 \%)$ patients complained of vertigo. Interestingly, 3 of 5 patients not reporting of hearing loss at presentation did show asymmetry on their first audiogram, none of the patients complaining of hearing loss revealed symmetrical hearing. Median VS size was $0.33 \mathrm{~cm}^{3}$ (range $\left.0.05-1.64\right)$. Twenty-one VS (58\%) were located on the right side and $15 \mathrm{VS}(42 \%)$ on the left.

\section{Predictors of change in audiologic function}

At the time of diagnosis, mean PTA was $49 \mathrm{~dB}$ and SDS was $68 \%$ on the affected side, compared with $25 \mathrm{~dB}$ and $95 \%$ on the non affected side $(P<0.001)$. The mean PTA and mean SDS on the affected side deteriorated significantly to $59.5 \mathrm{~dB}(P<0.001)$ and $57.9 \%(P<0.001)$ between the first and last audiogram. Hearing on the nonaffected side did not deteriorate significantly during FU: mean PTA remained 25dB and SDS decreased to $92 \%$ (Table 3.1). Ears presenting with labyrinthine hypointensity on T2-WI, showed significant higher deterioration of PTA over time compared to labyrinths maintaining isointense signal intensity $(P=0.01)$ (Table 3.2). Also a significant correlation between patient age and degree of hearing loss at presentation was seen, both in the affected as in the unaffected ear (PTA, P<0.003). Age, however did not influence the degree of hearing loss during FU significantly (Table 3.2). 
Table 3.1 The change in mean pure-tone average and speech discrimination score from diagnosis to end of follow-up, between the affected and nonaffected ear, calculated with the paired sample t-test.

\begin{tabular}{lccc} 
& $\begin{array}{c}\text { At Diagnosis } \\
\text { (standard deviation) }\end{array}$ & $\begin{array}{c}\text { End of FU } \\
\text { (standard deviation) }\end{array}$ & P-value \\
\hline Pure-tone average, affected ear & $49 \mathrm{~dB}(25)$ & $59.5 \mathrm{~dB}(27)$ & 0.001 \\
Speech discrimination score, affected ear & $68 \%(34)$ & $57.9 \%(34)$ & 0.02 \\
Pure-tone average, nonaffected ear & $25 \mathrm{~dB}(15)$ & $25 \mathrm{~dB}(17)$ & 0.66 \\
Speech discrimination score, nonaffected & $95 \%(13)$ & $92 \%(18)$ & 0.41
\end{tabular}

Table 3.2 Regression coefficients $(\beta)$ with 95\% confidence intervals from multivariate linear analyses on the effect of baseline characteristics on hearing loss during follow-up.

\begin{tabular}{|c|c|c|c|c|}
\hline Patient factors & $\begin{array}{l}\text { Change in pure-tone average } \\
\text { regression coefficient (95\% } \\
\text { confidence interval) }\end{array}$ & $\begin{array}{l}\mathrm{P}- \\
\text { value }\end{array}$ & $\begin{array}{l}\text { Change speech discrimination } \\
\text { score regression coefficient } \\
\text { (95\% confidence interval) }\end{array}$ & $\begin{array}{c}\mathrm{P}- \\
\text { value }\end{array}$ \\
\hline Age & $-0.2(-0.6-0.2)$ & 0.31 & $0.2(-0.3-0.7)$ & 0.45 \\
\hline Sex & & & & \\
\hline $\begin{array}{l}\text { Male }(n=17) \\
\text { Female }(n=19)\end{array}$ & $-1.1(-10.0-7.9)$ & 0.81 & $4.0(-6.7-14.7)$ & 0.45 \\
\hline Hearing complaint & & & & \\
\hline $\begin{array}{l}\text { No }(n=5) \\
\text { Yes }(n=31)\end{array}$ & $13.3(0.1-26.5)$ & $0.049^{*}$ & $-3.8(-19.8-12.3)$ & 0.66 \\
\hline Tinnitus & & & & \\
\hline $\begin{array}{l}\text { No }(n=24) \\
\text { Yes }(n=12)\end{array}$ & $-2.9(-11.1-5.2)$ & 0.50 & $-2.2(-12.1-7.7)$ & 0.67 \\
\hline Vertigo & & & & \\
\hline $\begin{array}{l}\text { No }(26) \\
\text { Yes }(10)\end{array}$ & $6.4(-2.3-15.2)$ & 0.14 & $5.3(-5.2-15.8)$ & 0.31 \\
\hline $\begin{array}{l}\text { Radiologic factors } \\
\text { VS Stage }\end{array}$ & & & & \\
\hline $\begin{array}{l}\text { A }(n=17) \\
B(n=15) \\
C(n=3) \\
D(n=1)\end{array}$ & $-1.6(-7.8-4.6)$ & 0.59 & $-1.4(-8.9-6.0)$ & 0.69 \\
\hline VS Side & & & & \\
\hline $\begin{array}{l}L(n=15) \\
R(n=21)\end{array}$ & $-2.8(11.1-5.5)$ & 0.49 & $-9.6(-19.6-0.5)$ & 0.07 \\
\hline $\begin{array}{l}\text { Hypointense signal } \\
\text { intensity labyrinth }\end{array}$ & & & & \\
\hline $\begin{array}{l}\text { No }(n=22) \\
\text { Yes }(n=14)\end{array}$ & $11.3(3.0-19.6)$ & $0.01^{*}$ & $-3.1(-13.4-7.1)$ & 0.54 \\
\hline $\begin{array}{l}\text { Significant growth } \\
\text { Yes }(n=15) \\
\text { No }(n=21)\end{array}$ & $-2.9(-11.5-5.7)$ & 0.50 & $-5.9(-14.3-3.9)$ & 0.26 \\
\hline
\end{tabular}

Mutual adjustment was performed for all variables in the table. Regression coefficients represent the mean difference in hearing loss between groups. Change in PTA=difference between first and last mean score on pure-tone audiogram (decibels). Change in SDS=difference between first and last speech discrimination score (\%). VS Stages: A=remaining in the internal auditory canal, $\mathrm{B}=$ extending into the cerebellopontine angle, $\mathrm{C}=$ compressing the brain stem, $\mathrm{D}=$ deviating the

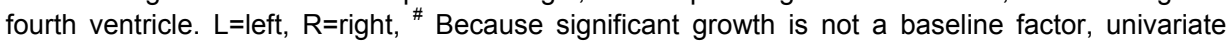
analysis was performed on this factor. ${ }^{*}=$ significant. 


\section{Predictors of changes in volume}

Over a median FU of 20.0 months (range 12-67 mo), 15 (41.6\%) of $36 \mathrm{VS}$ showed significant growth, whereas 21 VS $(58.3 \%)$ did not. Four of these nongrowing VS showed shrinkage. Of the 15 growing VS, 10 received intervention: 6 SRT $(60 \%)$ and 4 MS (40\%). One patient received SRT in absence of growth, because the patient preferred intervention, therefore 11 patients $(30.5 \%)$ received intervention. The other 5 patients showing growth remained in a W\&S policy, because it was the first FU scan on which growth was observed. The mean time period before intervention was 21.5 months (SD $13.4 \mathrm{mo}$ ). Patients who received intervention showed significant larger change in volume over time compared to the nonintervention group (30\% versus $5 \%$, $\mathrm{P}=0.02)$ and were significantly younger compared the W\&S counterpart $(56.2$ versus 63.9 respectively; $\mathrm{P}=0.01)$. Eight $(73 \%)$ of 11 patients receiving intervention, appeared with growth during the first FU year and six (24\%) of the 25 without intervention appeared with growth during the first $F U$ year $(P=0.01)$. Significant growth during the first year of FU was significantly related to further growth during follow up $(P=0.02)$, although significant growth during the first year of FU was not related to intervention during $F U(P=0.16)$. This paradox is caused by the fact that nonintervention in older patients with very slowly growing tumors complicated the calculation. No baseline factors were identified predicting growth (Table 3.3).

Table 3.3 Odds ratios (OR) with 95\% confidence intervals (Cl) from multivariate logistic analyses on the effect of baseline characteristics on significant growth during follow-up. Mutual adjustment was performed for all variables in the table. Odds ratio represents the strength of the association between a particular variable and probability of significant growth.

\begin{tabular}{|c|c|c|c|}
\hline Patient factors & & $\begin{array}{c}\text { Odds ratio ( } 95 \% \\
\text { confidence interval) } \\
\text { growth }\end{array}$ & P-value \\
\hline Age & $\begin{array}{l}<58.5 \text { year }(n=18) \\
>58.5 \text { year }(n=18)\end{array}$ & $0.50 \quad(0.08-2.72)$ & 0.40 \\
\hline Gender & $\begin{array}{l}\text { Male }(n=17) \\
\text { Female }(n=19)\end{array}$ & $1.29(0.60-29.9)$ & 0.77 \\
\hline Hearing complaint & $\begin{array}{l}\text { No }(n=5) \\
\text { Yes }(n=31)\end{array}$ & $1.02(0.06-16.4)$ & 0.99 \\
\hline Tinnitus & $\begin{array}{l}\text { No }(n=24) \\
\text { Yes }(n=12)\end{array}$ & $2.70 \quad(0.49-14.8)$ & 0.25 \\
\hline Vertigo & $\begin{array}{l}\text { No }(n=26) \\
\text { Yes }(n=10)\end{array}$ & $1.95 \quad(0.35-11.0)$ & 0.45 \\
\hline \multicolumn{4}{|l|}{ Radiological factors } \\
\hline VS Stage & $\begin{array}{l}A(n=17) \\
B-D(n=19)\end{array}$ & $4.48 \quad(0.61-29.9)$ & 0.15 \\
\hline VS Side & $\begin{array}{l}L(n=15) \\
R(n=21)\end{array}$ & $3.67 \quad(0.67-22.2)$ & 0.13 \\
\hline Hypointense signal intensity labyrinth & $\begin{array}{l}\text { No }(n=22) \\
\text { Yes }(n=14)\end{array}$ & $2.01 \quad(0.41-11.0)$ & 0.34 \\
\hline
\end{tabular}




\section{Increase in volume related to audiologic deterioration}

Multivariate linear analysis was performed on the relationship between increase in volume and hearing deterioration, in which we adjusted for the signal intensity of the affected labyrinth and the presence of intervention during the W\&S policy. No significant correlation was found between increase in volume and change in hearing function. The presence of significant growth was not associated with a significant difference in hearing loss when compared with stable VS (Table 3.2).

\section{Discussion}

The natural history of VS remains unclear, with percentages of tumors showing evidence of growth varying from 30 to $90 \%$, depending at least in part on the length of the observation period ${ }^{1-3}$. In a W\&S policy, containment of VS growth and maintenance of functional hearing are the major goals. Therefore, factors predicting growth and hearing loss in these patients would be invaluable. This study presents the data obtained after analyzing patients managed purely conservatively. For measuring VS size, a novel measuring tool was used, superior to 2-dimensional measurements ${ }^{12}$, used in previous studies , $^{2,3,7-10,13-28}$. As per a prior publication ${ }^{12}$, volume measurements are a more reproducible way to assess tumor growth, in contrast to 2-dimensional studies in which a 1 or 2 millimeter difference between subsequent scans was arbitrarily defined as growth ${ }^{2,3,7-10,13-28}$. Two-dimensional tumor measurement differences of 1 to 2 $\mathrm{mm}$ lie within measurement error as calculations with 3-dimensional measurements show ${ }^{12}$. To our best knowledge, this study is the first to use volume measurements and a statistically justified definition of significant growth to correlate growth and loss in audiologic function with baseline characteristics. Therefore, this study is expected to be more sensitive for detecting correlations, if they exist.

\section{Predictors of change in audiologic function}

Patients with complaints of hearing loss at diagnosis appear with a larger increase in PTA threshold during FU $(P=0.049)$. This is in agreement with recent findings of Stangerup et al. ${ }^{11}$ : established hearing loss is a strong predictor of further hearing loss, whereas patients with minimal PTA losses and an SDS of $100 \%$ are unlikely to loose their functional hearing.

The prognostic value of the labyrinthine signal intensity, measured on T2-WI MRI, for hearing loss in VS patients during FU in a W\&S policy, is presented for the first time. Somers et al. ${ }^{30}$ found that hypointense labyrinths were associated with a worse hearing outcome after hearing preservation VS 
surgery efforts. In this study, a significant difference in hearing loss could be demonstrated between FU in hypointense and isointense labyrinths $(P=0.01)$. This implies that whenever the labyrinth is hypointense on T2-WI, hearing worsens at a faster rate compared to isointense labyrinths. The exact cause of the diminished signal intensity is not well understood. Previous studies indicate that the presence of a VS causes significant biochemical alterations of the inner ear fluids ${ }^{31-33}$, with an increased perilymphatic protein content ${ }^{33,34}$. It has been suggested that these alterations are related to vascular impairment caused by mechanical obstruction by the $\mathrm{VS}^{30,31}$. This hypothesis may be supported by Lapsiwala et al. ${ }^{35}$, who found a significant correlation between IAC pressure and preoperative hearing level. This finding is specifically important when patients, who are still candidates for hearing preservation surgery or SRT, are considered for entering a W\&S policy. In patients who present with a hypointense labyrinth, with a wish to preserve hearing, active intervention may be preferable, because of a significant increased risk of further hearing deterioration.

VS side and VS stage showed no significant correlation with audiologic deterioration, similar to findings in literature ${ }^{8,9,17,18,36}$. Increasing age showed a significant relationship with worse hearing, both in the VS affected as in the unaffected group. This relation is due to a presbyacusis effect, which is present in both ears. Age, however, did not influence the degree of hearing loss over time in affected ears. Sex did not provide significant correlations with auditory function. According to our findings, only hearing loss at diagnosis and labyrinthine hypointensity are predictors of further hearing loss.

\section{Predictors of changes in volume}

Significant growth during the first year of FU was related significantly to growth during FU $(P=0.02)$. This relationship has been found before, although in all previous studies growth was defined as a predefined increase in VS diameter and not as a volume measure ${ }^{3,25,37}$.

None of the baseline characteristics, which were evaluated in this study, predicted growth. These findings are in agreement with preceding publications $s^{2,3,9,10,14-16,23,25}$, although only two studies used volume measurements ${ }^{38,39}$. In other studies, some baseline characteristics have been reported to be positively associated with VS growth, such as duration of symptoms greater than 10 years and tinnitus at presentation ${ }^{14}$, VS diameter greater than $20 \mathrm{~mm}^{16}$ and VS extending in the $\mathrm{CPA}^{7,8,18}$. One study found progressive sensorineural hearing loss at presentation to be associated with significant less VS growth during FU, compared with patients presenting with tinnitus or vertigo ${ }^{25}$. All of these prior studies used less reliable 2-dimensional measurements in their analysis. Apart from duration of symptoms and VS 
diameters, which were not assessed in this study, these relations could not be reproduced.

Significant VS growth was found in 15 (41.6\%) of 36 patients during FU. In 11 $(30.5 \%)$ of 36 patients, intervention was necessary. No factors could be identified which predict the need for intervention. This finding is in line with previous publications ${ }^{7,8,18}$, although some authors found that growth during the first year of FU was significantly associated with the need for intervention during $\mathrm{FU}^{37}$. Others reported that conservative management failed more often in patients who presented with vertigo ${ }^{19}$. According to our results, we can conclude that growth is not predictable by any information present at diagnosis and that growth during the first year of FU is a prognostic factor of further VS growth.

\section{Volume change as predictor of change in audiologic function}

By using precise volume measurements, changes in VS volume were compared with change in audiologic function. Increase in VS volume did not correlate significantly with a change in PTA or SDS. Hearing deteriorates whether the VS grows or not, which agrees with the conclusion of other authors $^{7,9,17,18,25,28}$, relying on 2-dimensional measurements with unreliable growth cut off points. Other 2-dimensional studies found "significant growth" related to a significant higher $\mathrm{PTA}^{7,9,18,20,21,28}$ and worse $\mathrm{SDS}^{9,18}$ during $\mathrm{FU}$, while others could not prove this for both $\mathrm{PTA}^{8,24,25,27}$ and $\mathrm{SDS}^{7,8,17,25,27,28}$. Few authors performed volume measurements related to hearing deterioration ${ }^{39,40}$. One study calculated VS volume for purely intracanalicular schwannomas with specific formulas ${ }^{40}$, which is known to produce large overestimations of tumor $\mathrm{size}^{23}$. They found absolute volume growth rate to be positively related to PTA deterioration and loss of PTA was significantly smaller in shrinking VS. Massick et al. ${ }^{39}$ found a significant correlation between change in VS volume and change in PTA and SDS; however the number of included patients was low and their measurement technique was not specified.

The finding that growth is not associated with change in audiologic function supports the hypothesis that hearing loss in VS patients is not mainly caused by compression of the cochlear nerve. Other factors might be involved, for example differences in biological VS behaviour, compromise in vascular supply of the cochlear nerve and cochlea and biochemical alterations or loss of inner ear fluids ${ }^{31-33}$. The latter hypothesis is supported by our finding that hearing loss occurs more often in patients showing a hypointense signal intensity on T2-WI.

Although the study findings do not directly support using volume measurements during FU of VS, we recommend volume measurements of VS as the most reliable way to assess whether a tumor is growing, where tumor 
growth is to be a factor in choosing whether to intervene ${ }^{12}$. Sequential MRI for determining VS growth remains the golden standard and can therefore not be replaced by audiologic examination solely.

The study had some limitations. Data on symptoms were recorded retrospectively, the number of patients with follow up MRI/audiogram combinations is low and the FU period is relatively short. For this reason, the study may not have been sufficiently powered to detect small but relevant associations between patient or VS related features baseline characteristics and changes in volume and audiologic function during FU.

\section{Conclusion}

In this study, patient and VS-related risk factors for growth or hearing loss were determined for patients followed in a W\&S policy, in which a novel volumetric measuring tool was used. Patients whose presenting complaints included hearing loss did indeed have significant worse hearing than those who were unaware of hearing loss at time of tumor diagnosis. Hypointensity of the affected labyrinth on T2-WI at diagnosis results in a significantly faster deterioration of PTA which may argue in favor of early intervention if hearing salvage is under consideration. Audiologic deterioration occurs irrespective of VS growth and growth during the first year of FU predicts further growth during FU. Volume measurements are not a reliable measure predicting auditory function and vice versa. No other factors predicting growth were identified; therefore, sequential MRI remains the only objective method for monitoring of VS growth during a W\&S policy. 


\section{References}

1. Charabi S, Tos M, Thomsen J, Charabi B, Mantoni M. Vestibular schwannoma growth: the continuing controversy. Laryngoscope. 2000;110(10 Pt 1):1720-5.

2. Stangerup SE, Caye-Thomasen $P$, Tos $M$, Thomsen J. The natural history of vestibular schwannoma. Otol Neurotol. 2006;27:547-52.

3. Smouha EE, Yoo M, Mohr K, Davis RP. Conservative management of acoustic neuroma: a meta-analysis and proposed treatment algorithm. Laryngoscope. 2005; 115:450-4.

4. Hasegawa T, Fujitani S, Katsumata S, Kida Y, Yoshimoto M, Koike J. Stereotactic radiosurgery for vestibular schwannomas: analysis of 317 patients followed more than 5 years. Neurosurgery. 2005;57:257-65.

5. Kondziolka D, Lunsford LD, McLaughlin MR, Flickinger JC. Long-term outcomes after radiosurgery for acoustic neuromas. The New England journal of medicine. 1998;339:1426-33.

6. Lunsford LD, Niranjan A, Flickinger JC, Maitz A, Kondziolka D. Radiosurgery of vestibular schwannomas: summary of experience in 829 cases. Journal of neurosurgery. 2005;102 Suppl:195-9.

7. Raut VV, Walsh RM, Bath AP, Bance ML, Guha A, Tator CH, Rutka JA. Conservative management of vestibular schwannomas - second review of a prospective longitudinal study. Clin Otolaryngol Allied Sci. 2004;29:505-14.

8. Walsh RM, Bath AP, Bance ML, Keller A, Tator $\mathrm{CH}$, Rutka JA. The role of conservative management of vestibular schwannomas. Clin Otolaryngol Allied Sci. 2000;25:28-39.

9. Stangerup SE, Caye-Thomasen $\mathrm{P}$, Tos M, Thomsen J. Change in hearing during 'wait and scan' management of patients with vestibular schwannoma. J Laryngol Otol. 2008;122:673-81.

10. Bakkouri WE, Kania RE, Guichard JP, Lot G, Herman P, Huy PT. Conservative management of 386 cases of unilateral vestibular schwannoma: tumor growth and consequences for treatment. Journal of Neurosurgery. 2009;110:662-9.

11. Stangerup SE, Tos M, Thomsen J, Caye-Thomasen P. Hearing outcomes of vestibular schwannoma patients managed with 'wait and scan': predictive value of hearing level at diagnosis. J Laryngol Otol. 2010;124:490-4.

12. van de Langenberg R, de Bondt BJ, Nelemans PJ, Baumert BG, Stokroos RJ. Follow-up assessment of vestibular schwannomas: volume quantification versus two-dimensional measurements. Neuroradiology. 2009;51:517-24.

13. Berrettini S, Ravecca F, Sellari-Franceschini S, Bruschini P, Casani A, Padolecchia R. Acoustic neuroma: correlations between morphology and otoneurological manifestations. J Neurol Sci. 1996;144:24-33.

14. Ferri GG, Modugno GC, Pirodda A, Fioravanti A, Calbucci F, Ceroni AR. Conservative management of vestibular schwannomas: an effective strategy. Laryngoscope. 2008;118:951-7.

15. Flint D, Fagan $P$, Panarese A. Conservative management of sporadic unilateral acoustic neuromas. J Laryngol Otol. 2005;119:424-8.

16. Fucci MJ, Buchman CA, Brackmann DE, Berliner KI. Acoustic tumor growth: implications for treatment choices. Am J Otol. 1999;20:495-9.

17. Graamans K, Van Dijk JE, Janssen LW. Hearing deterioration in patients with a non-growing vestibular schwannoma. Acta Otolaryngol. 2003;123:51-4. 
18. Hajioff D, Raut VV, Walsh RM, Bath AP, Bance ML, Guha A, Tator CH, Rutka JA. Conservative management of vestibular schwannomas: third review of a 10-year prospective study. Clin Otolaryngol. 2008;33:255-9.

19. Malhotra PS, Sharma P, Fishman MA, Grumbine FL, Tholey R, Dam VQ, Dasgupta A, Pequignot E, Willcox TO. Clinical, radiographic, and audiometric predictors in conservative management of vestibular schwannoma. Otol Neurotol. 2009;30: 507-14.

20. Nadol JB, Jr., Diamond PF, Thornton AR. Correlation of hearing loss and radiologic dimensions of vestibular schwannomas (acoustic Neuromas). Am J Otol. 1996;17: 312-6.

21. Rosenberg SI. Natural history of acoustic neuromas. Laryngoscope. 2000;110: 497-508.

22. Sakamoto T, Fukuda S, Inuyama Y. Hearing loss and growth rate of acoustic neuromas in follow-up observation policy. Auris Nasus Larynx. 2001;28 Suppl: S23-7.

23. Shin YJ, Fraysse B, Cognard C, Gafsi I, Charlet JP, Berges C, Deguine O, Tremoulet M. Effectiveness of conservative management of acoustic neuromas. Am J Otol. 2000;21:857-62.

24. Tierney PA, Chitnavis BP, Sherriff M, Strong AJ, Gleeson MJ. The relationship between pure tone thresholds and the radiological dimensions of acoustic neuromas. Skull Base Surg. 1998;8:149-51.

25. Tschudi DC, Linder TE, Fisch U. Conservative management of unilateral acoustic neuromas. Am J Otol. 2000;21:722-8.

26. Van Dijk JE, Duijndam J, Graamans K. Acoustic neuroma: deterioration of speech discrimination related to thresholds in pure-tone audiometry. Acta Otolaryngol. 2000;120:627-32.

27. van Leeuwen JP, Cremers CW, Thewissen NP, Harhangi BS, Meijer E. Acoustic neuroma: correlation among tumor size, symptoms, and patient age. Laryngoscope. 1995;105:701-7.

28. Walsh RM, Bath AP, Bance ML, Keller A, Rutka JA. Consequences to hearing during the conservative management of vestibular schwannomas. Laryngoscope. 2000;110:250-5.

29. Committee on Hearing and Equilibrium guidelines for the evaluation of hearing preservation in acoustic neuroma (vestibular schwannoma). American Academy of Otolaryngology-Head and Neck Surgery Foundation, INC. Otolaryngol Head Neck Surg. 1995;113:179-80.

30. Somers T, Casselman J, de Ceulaer G, Govaerts P, Offeciers E. Prognostic value of magnetic resonance imaging findings in hearing preservation surgery for vestibular schwannoma. Otol Neurotol. 2001;22:87-94.

31. O'Connor AF, Luxon LM, Shortman RC, Thompson EJ, Morrison AW. Electrophoretic separation and identification of perilymph proteins in cases of acoustic neuroma. Acta Otolaryngol. 1982;93:195-200.

32. Pialoux P, Freyss G, Trotoux J. [Study of the physico-chemical components of the labyrinthine fluids in diseases of the inner ear. Diagnostic value. 1st results]. Ann Otolaryngol Chir Cervicofac. 1972;89:385-96.

33. Silverstein $\mathrm{H}$, Schuknecht HF. Biochemical studies of inner ear fluid in man. Changes in otosclerosis, Meniere's disease, and acoustic neuroma. Arch Otolaryngol. 1966;84:395-402.

34. Johnsson LG, Hawkins JE, Jr., Rouse RC. Sensorineural and vascular changes in an ear with acoustic neurinoma. Am J Otolaryngol. 1984;5:49-59. 
35. Lapsiwala SB, Pyle GM, Kaemmerle AW, Sasse FJ, Badie B. Correlation between auditory function and internal auditory canal pressure in patients with vestibular schwannomas. Journal of neurosurgery. 2002;96:872-6.

36. Gerganov V, Nouri M, Stieglitz L, Giordano M, Samii M, Samii A. Radiological factors related to pre-operative hearing levels in patients with vestibular schwannomas. J Clin Neurosci. 2009;16:1009-12.

37. Deen HG, Ebersold MJ, Harner SG, Beatty CW, Marion MS, Wharen RE, Green JD, Quast L. Conservative management of acoustic neuroma: an outcome study. Neurosurgery. 1996;39:260-4.

38. Herwadker A, Vokurka EA, Evans DG, Ramsden RT, Jackson A. Size and growth rate of sporadic vestibular schwannoma: predictive value of information available at presentation. Otol Neurotol. 2005;26:86-92.

39. Massick DD, Welling DB, Dodson EE, Scholfield M, Nagaraja HN, Schmalbrock P, Chakeres DW. Tumor growth and audiometric change in vestibular schwannomas managed conservatively. Laryngoscope. 2000;110:1843-9.

40. Caye-Thomasen P, Dethloff T, Hansen S, Stangerup SE, Thomsen J. Hearing in patients with intracanalicular vestibular schwannomas. Audiol Neurootol. 2007;12: 1-12. 



\section{VOLUME CHANGES AFTER STEREOTACTIC LINAC RADIOTHERAPY IN VESTIBULAR SCHWANNOMA: CONTROL RATE AND GROWTH PATTERNS}

Rick van de Langenberg Amy JC Dohmen

Bert J de Bondt Patty J Nelemans Brigitta G Baumert Robert J Stokroos

International Journal Radiation Oncology * Biology * Physics. 2012;84:343-49 


\section{Abstract}

\section{Purpose}

The purpose of this study was to evaluate the control rate of vestibular schwannomas (VS) after treatment with linear accelerator (LINAC) based stereotactic radiosurgery (SRS) or radiotherapy (SRT), by using a validated volumetric measuring tool. Volume based studies on prognosis after LINAC based SRS or SRT for VS are reported scarcely. In addition, growth patterns and risk factors predicting treatment failure were analyzed.

\section{Materials and Methods}

Retrospectively, 37 VS patients were analyzed, treated with LINAC based SRS or SRT. Baseline and follow-up MRI were analyzed with volume measurements on contrast enhanced T1-weighted MRI. Absence of intervention after radiotherapy was defined as "no additional intervention group", absence of radiological growth was defined as "radiological control group". Significant growth was defined as a volume change of $19.7 \%$ or more, as calculated in a previous study.

\section{Results}

The cumulative 4-year probability of no additional intervention was $96.4 \%( \pm 0.03)$, the 4-year radiological control probability was $85.4 \%( \pm 0.1)$. The median follow-up was 40 months. Overall, shrinkage was seen in $65 \%$, stable VS in $22 \%$ and growth in $13 \%$. In $54 \%$ of all patients transient swelling was observed. No prognostic factors were found regarding VS growth. Previous treatment and SRS were associated with transient swelling significantly.

\section{Conclusions}

Good control rates are reported for LINAC based SRS or SRT in VS, in which the lower rate of radiological growth control is attributed to the use of the more sensitive volume measurements. Transient swelling after radiosurgery is a common phenomenon and should not be mistaken for treatment failure. Previous treatment and SRS were associated with transient swelling significantly. 


\section{Introduction}

A vestibular schwannoma (VS) is a benign tumor originating from the Schwann cells of the 8th cranial nerve. Incidence is increasing, mostly due to aging of the population and increasing use and improvement of magnetic resonance imaging $(\mathrm{MRI})^{1}$.

Three main treatment modalities exist in VS management: a so called "wait and scan" (W\&S) policy, in which audiovestibular symptoms are monitored regularly combined with sequential MRI to follow VS growth, microsurgery or stereotactic radiotherapy ${ }^{2}$. The latter has proven to be a well-established and safe alternative to microsurgery for small and medium sized VS, with lower morbidity and similar rates of $\mathrm{VS}$ control $^{3}$.

Many studies reported on control rate after radiotherapy for VS. An important limitation of these studies is that measurements are mostly based on twodimensions $(2 D)^{2,4-31}$ instead of volume measurements ${ }^{31-38}$, and are therefore not ideal in assessing VS growth precisely ${ }^{39}$. Another limitation is that these 2D-studies use arbitrary growth criteria of 1 or $2 \mathrm{~mm}^{2,5,6,10,13-16,20,25,27}$, or do not even mention these growth cut off points ${ }^{4,9,11,12,17,19,21,22,26,28,30,31,40}$. According to a previous study, these cut off points lie within the measurement error ${ }^{39}$, making the results of these studies questionable. Two articles used volume measurements after linear accelerator (LINAC) radiosurgery ${ }^{33,35}$. The control rate of VS after LINAC stereotactic radiosurgery (SRS) or radiotherapy (SRT) was reassessed in this study, by using a more precise assessment of VS growth with a validated volumetric measuring tool and previously defined growth cut-off points for this technique ${ }^{39}$. Furthermore, growth patterns were analyzed and risk factors predicting postradiosurgical VS growth were assessed.

\section{Patients and methods}

\section{Patients characteristics}

Patients who received LINAC based SRS or SRT for a VS between February 2003 and March 2008 in the department of the Maastricht Radiation-Oncology (MAASTRO-clinic), Maastricht University Medical Center were analyzed retrospectively in this study, resulting in 40 patients. One patient with neurofibromatosis type 2 was excluded. Two patients were excluded because they had only one follow-up (FU) MRI. Thirty-two patients were irradiated as primary treatment after being followed in a W\&S policy. Seven patients received radiotherapy after subtotal VS resection. Histopathological examination showed VS in five cases. Two cases appeared to be a meningeoma, for which they were excluded from this study. Therefore, the final 
analysis was based on 37 patients. Because this study was an analysis of radiological data, we did not include clinical FU parameters routinely for all patients. Clearly, in cases in which additional treatment was necessary, both radiological progression and clinical symptoms were taken into account and, if present, included in the present study.

\section{Stereotactic radiotherapy}

All patients were treated with LINAC-based stereotactic radiotherapy. Stereotactic radiotherapy was given either in a single session (stereotactic radiosurgery, SRS) or in a fractionated manner (SRT). Seventeen patients received SRS with 12 or $12.5 \mathrm{~Gy}$ prescribed to the $80 \%$ isodose line covering the VS margin. The other 20 patients received fractionated SRT with 30 fractions of $1.8 \mathrm{~Gy}$ to a total dose of $54 \mathrm{~Gy}$ (Table 4.1). SRT was prescribed to the $100 \%$ according to ICRU guidelines ${ }^{41}$. The technique of stereotactic radiotherapy has been described in detail previously ${ }^{42}$. Indications for LINAC based SRS or SRT were mainly clinical and/or radiological progression of the VS, postoperative radiotherapy in order to avoid further progression or primary irradiation in case of co-morbidity. SRS was applied in general when the VS was smaller than $2 \mathrm{~cm}$ in largest diameter, had no close contact to the brainstem or if useful hearing was absent. In case of larger VS, the presence of (close, $<2 \mathrm{~mm}$ ) contact to the brainstem as well as the presence of useful hearing on the side of the VS, fractionated SRT was chosen. Patient-related factors as old age, comorbidity or long travel distance were indicators for SRS.

Table 4.1 Patient and vestibular schwannoma characteristics $(n=37)$.

\begin{tabular}{|c|c|c|}
\hline \multicolumn{2}{|c|}{ Patient or VS Characteristic } & Number (\%) \\
\hline \multirow[t]{2}{*}{ Sex } & Male & $16(43)$ \\
\hline & Female & $21(57)$ \\
\hline \multirow[t]{2}{*}{ Age (year) } & Mean & 61.6 \\
\hline & Range & $37-81$ \\
\hline \multirow[t]{2}{*}{ Side } & Left & $22(59)$ \\
\hline & Right & $15(41)$ \\
\hline \multirow[t]{2}{*}{ Radiotherapy } & Single & $17(46)$ \\
\hline & Fractionated & $20(54)$ \\
\hline \multirow[t]{2}{*}{ Follow-up (months) } & Median & 40 \\
\hline & Range & $16-79.5$ \\
\hline \multicolumn{2}{|c|}{ Previous microsurgery } & $5(14)$ \\
\hline
\end{tabular}

\section{Follow-up}

Follow-up was performed with a first MRI scan 3 months after SRS/SRT (baseline scan) and yearly thereafter until 5 years after treatment. Thereafter, once every other year, scans are performed until at least 10 years of FU are reached. In case of radiological progression or clinical symptoms, a more frequent FU was used. Two hundred and five scans were available for analysis. 


\section{Methods}

MRI sequences included 1 or 2 -mm thick contrast-enhanced T1 weighted images (CE-T1 WI). The volume measurements were performed on axial coupes, using a computer system fitted with specialized software: iPlan ${ }^{2}$ RTImage version 4 (BrainLab Oncology Solutions, Feldkirchen, Germany). By tracing the VS surface on all slices the software was able to provide the VS volume by using the slice interval. Segmentation results were checked visually. Measurements typically took a few minutes. VS characteristics were noted: central nonenhancement was defined as a hypointense mass in the center, on CE-T1 WI. Cystic VS was defined as a VS with enhanced cysts making up at least one-third of nonenhancement of the VS volume on CE-T1 WI, and enhancing on T2 weighted images. Volumetric VS analysis was performed in cubic centimetre $\left(\mathrm{cm}^{3}\right)$. In all patients a pre-treatment MRI imaging protocol (3D scan with $1 \mathrm{~mm}$ voxels) was used to evaluate VS size before SRS/SRT start. Baseline VS size was defined as volume on the last pre-treatment MRI. VS growth rates were calculated for each FU. The growth patterns were visualised by plotting the change VS volume at each FU point against the FU time. Further technical details regarding MRI, the parameters of the sequences used, can be found in a previous publication, in which the volume measuring method used in the present study was validated ${ }^{39}$. In short, the maximum interobserver measurement error was calculated for a large set of VS. "Real" growth, beyond measurement error, can be found when growth exceeds this measurement error, which was $19.7 \%$. Therefore, significant growth was defined as a percentage increase in volume that exceeded $19.7 \%$. Significant shrinkage was defined as a percentage decrease in volume exceeding $19.7 \%$. When no significant growth or shrinkage was seen, these VS were called stable. Transient swelling was defined as shrinkage preceded by significant growth after radiotherapy. This shrinkage had to regress to baseline size before it was called "transient swelling", otherwise it was classified as growth.

The "radiological control group" included the VS in which no significant growth on the last FU scan was seen. Absence of intervention after SRS/SRT, which was either microsurgery (MS) or second radiotherapy (SRS/SRT), was defined as the "no additional intervention group". The decision to perform additional intervention was taken in a multidisciplinary team meeting. Additional intervention was necessary after diagnosing continuing growth or uncontrollable clinical symptoms. Radiological definition of continuing growth was based on at least 2 repeated MRI scans which both demonstrated growth. In the first two years after treatment growth was accepted because of the chance of transient swelling. Uncontrollable clinical symptoms are symptoms which are not treatable by adapted medication or otherwise and were not observed in our group. 


\section{Statistical analysis}

Differences in mean values were tested for significance using the independent samples t-test for comparison between two groups and using analysis of variance (ANOVA) for comparison between more than two groups. Fisher exact and $\chi^{2}$ test were used for categorical data. A Cox proportional hazards model was used to analyse hazard ratios (HR) for patient, treatment and VS characteristics on the occurrence of additional intervention, radiological failure and transient swelling. The cumulative probability of no additional intervention and radiological control with $95 \%-\mathrm{Cl}$ was calculated using Kaplan-Meier analyses. P-values $<0.05$ were considered to indicate statistical significance. All analyses were performed using SPSS15.0 statistical software (SPSS, Chicago, II, USA).

\section{Results}

\section{Patient characteristics}

There were 37 patients, 16 men and 21 women, with a mean age of 61.6 years (range 37-81). VS was right sided in 15 patients and left sided in 22 patients. VS volume ranged from 0.07 to $16.28 \mathrm{~cm}^{3}$ (mean $2.09 \mathrm{~cm}^{3}$ ). The median FU was 40.0 (16-79.5) months (Table 4.1).

\section{VS control rate}

\section{Radiological control group}

The 4-year radiological control probability was $85.4 \%( \pm 0,1)$ (Table 4.2, Figure 4.1). When comparing volumes of the last FU MRI with baseline MRI, significant VS shrinkage was seen in 24 patients (65\%), stable VS in 8 patients $(22 \%)$ and significant growth in 5 patients $(13 \%)$. Two of the 5 patients with significant growth showed continuous growth during FU. Two other patients showed transient swelling followed by regrowth, remaining stable thereafter. One patient showed transient swelling at last FU (16 months). Because this volume was still significantly above the baseline volume, this patient was included in the growth group. 
Table 4.2 Cumulative probability of survival without radiological failure during follow-up after LINAC radiosurgery of vestibular schwannoma.

\begin{tabular}{|lccc|}
\hline Follow-up (months) & Number at risk & Cumulative probability of survival (\%) & $95 \% \mathrm{Cl}$ \\
\hline 12 & 35 & 94.5 & $80.1-98.6$ \\
24 & 28 & 88.8 & $72.8-95.6$ \\
36 & 23 & 85.4 & $68.2-93.7$ \\
48 & 15 & 85.4 & $68.2-93.7$
\end{tabular}

$\mathrm{Cl}=$ confidence interval.

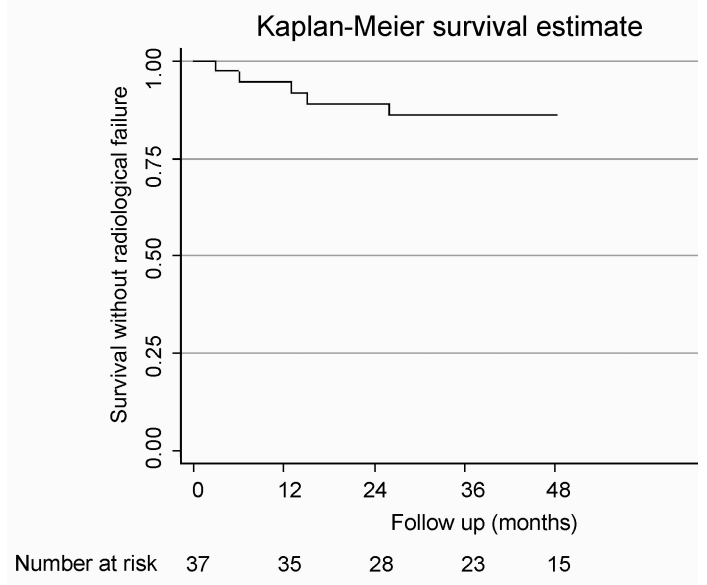

Figure 4.1 Cumulative probability of survival without radiological failure during FU after LINAC radiosurgery of vestibular schwannoma.

No additional intervention group

The 4-year probability of absence of additional treatment was $96.4 \%( \pm 0.03)$ (Table 4.3, Figure 4.2). Of the five patients showing growth, one patient with continuous growth received microsurgery 32 months after treatment. The other patient with continuous growth refused further intervention and remains in a W\&S policy, like the remaining three patients. These patients did not suffer from clinical symptoms accompanying VS growth. Another patient received microsurgery five years after SRT, because of late regrowth of VS volume from $50 \%$ to $108 \%$ after 60 months. 
Table 4.3 Cumulative probability of survival without additional intervention during follow-up after LINAC radiosurgery of vestibular schwannoma.

\begin{tabular}{|cccc} 
Follow-up & Number at risk & Cumulative probability of survival (\%) & $95 \% \mathrm{Cl}$ \\
\hline 12 & 37 & 100 & - \\
24 & 31 & 100 & - \\
36 & 24 & 96.4 & $77.2-99.5$ \\
48 & 15 & 96.4 & $77.2-99.5$
\end{tabular}

$\mathrm{Cl}=$ confidence interval.

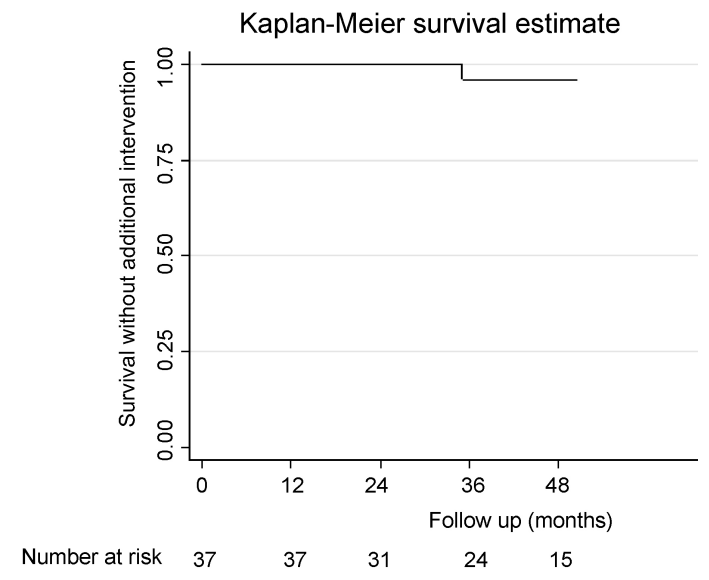

Figure 4.2 Cumulative probability of survival without additional intervention during FU after LINAC radiosurgery of vestibular schwannoma.

\section{Growth patterns}

Different types of growth patterns were identified during $\mathrm{FU}$ and classified into three types (Figure 4.3). 1) Growth (13\%), which was further subdivided in continuous growth $(5 \%, 2 / 37)$, transient swelling followed by late regrowth $(5 \%$, $2 / 37)$ and transient swelling followed by shrinkage $(3 \%, 1 / 37) .2)$ Shrinkage $(65 \%)$, which was subdivided in direct shrinkage $(35 \%, 13 / 37)$ and transient swelling followed by shrinkage $(30 \%, 11 / 37)$. 3) Stable VS $(22 \%)$, which was subdivided in stable VS $(5 \%, 2 / 37)$, transient swelling followed by shrinkage $(14 \%, 5 / 37)$, and transient swelling followed by late regrowth $(3 \%, 1 / 37)$.

VS showing transient swelling $(n=20,54 \%)$ reached a maximum volume after a median of 5 months (range 3-17 months) and first measured regression was seen at a median of 15 months (range 8-27 months). VS regressed beyond baseline volume after transient swelling $(n=16)$ in a median of 24 months (range 11-62 months). Late regrowth occurred at a median of 27.5 months (range 26.5-53 months). 

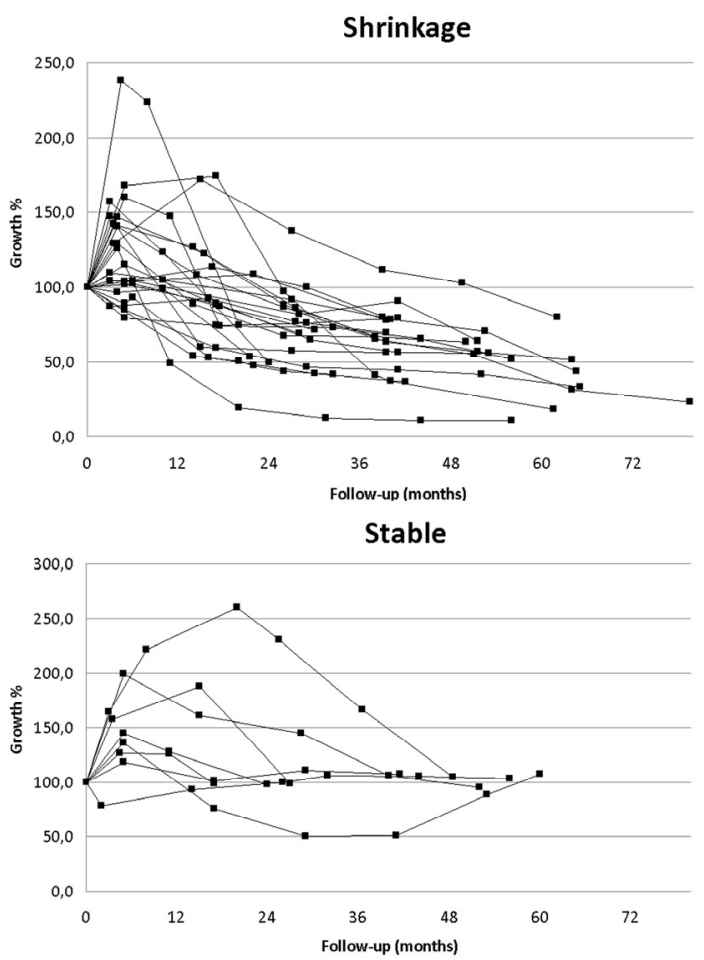

Growth

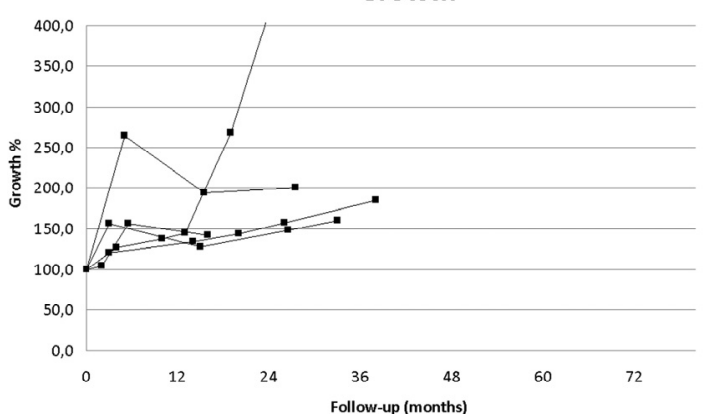

Figure 4.3 Graphs visualizing the percentage change in VS volume during follow-up compared to the baseline MRI. The VS was classified as "shrinkage", "growth and "stable".

\section{Central nonenhancement and cysts}

Central nonenhancement was seen in 22 patients (59\%) during FU, 9 patients $(24 \%)$ showed no central nonenhancement and 6 patients $(16 \%)$ showed cysts at baseline scan.

The onset of central nonenhancement ranged from 0 to 6 months (median 3.5 months) and was last seen between 15.5 to 61.5 months (median 22 months) 
after SRS or SRT. Central nonenhancement disappeared during FU in 27.5 to 48.5 months (median 29.5 months) after SRS or SRT and was still present at last FU MRI in 12 patients (12/22, 55\%) at 16 to 64.5 months (median 39.5).

VS with central nonenhancement did not show a larger amount of maximum volume increase compared to VS without central nonenhancement $(P=0.389)$. Furthermore, baseline cystic VS showed no significant larger amount of volume increase compared to VS without cystic components $(P=0.399)$.

\section{Prognostic factors}

Specific factors were analyzed in order to find factors predicting transient swelling, radiological failure or additional intervention. Because of the limited amount of patients receiving additional intervention, no risk factors could be calculated. No factors could be identified for radiological failure. Treatment before SRS/SRT and treatment with SRS was associated with a larger risk of transient swelling significantly (Table 4.4).

Table 4.4 Risk factors VS control.

A Cox proportional hazards model, used to calculate hazard ratios (HR) for patient and VS characteristics on the occurrence of additional intervention, radiological failure and transient swelling.

\begin{tabular}{|c|c|c|c|c|c|c|c|}
\hline & $\begin{array}{c}\text { Additional } \\
\text { intervention } \\
(\mathrm{HR})\end{array}$ & $\begin{array}{l}\text { Radiological } \\
\text { failure (HR) }\end{array}$ & $95 \% \mathrm{Cl}$ & P-value & $\begin{array}{c}\text { Transient } \\
\text { swelling } \\
\text { (HR) }\end{array}$ & $95 \% \mathrm{Cl}$ & P-value \\
\hline Sex (male/female) & NR & 2.120 & $0.25-18.00$ & 0.491 & 1.198 & $0.37-3.88$ & 0.763 \\
\hline Age $(<60 />60)$ & NR & 0.639 & $0.10-4.10$ & 0.636 & 2.195 & $0.81-6.00$ & 0.124 \\
\hline $\begin{array}{l}\text { Location } \\
\text { (left/right) }\end{array}$ & NR & 3.639 & $0.45-29.37$ & 0.225 & 1.112 & $0.35-3.55$ & 0.858 \\
\hline $\begin{array}{l}\text { Previous } \\
\text { treatment (no/yes) }\end{array}$ & NR & NR & NR & NR & 5.616 & $1.16-27.12$ & $0.032^{*}$ \\
\hline SRS/SRT & NR & 0.300 & $0.04-2.26$ & 0.242 & 0.278 & $0.08-0.96$ & $0.043^{*}$ \\
\hline $\begin{array}{l}\text { Volume } \\
\left(<1 />1 \mathrm{~cm}^{3}\right)\end{array}$ & NR & 0.663 & $0.05-8.18$ & 0.749 & 0.544 & $0.17-1.72$ & 0.301 \\
\hline $\begin{array}{l}\text { Central } \\
\text { nonenhancement } \\
\text { (no/yes) }\end{array}$ & NR & 3.417 & $0.29-40.04$ & 0.328 & 2.589 & $0.57-11.7$ & 0.216 \\
\hline Cyst (no/yes) & NR & NR & NR & NR & 2.040 & $0.46-11.46$ & 0.418 \\
\hline
\end{tabular}

W\&S=wait and scan policy. $\mathrm{Cl}=$ confidence interval. SRS=stereotactic radiosurgery. $\mathrm{SRT}=$ stereotactic radiotherapy. NR: not enough events for results. ${ }^{*}$ is significant $(\mathrm{P}<0.05)$.

\section{Discussion}

\section{VS control rates}

The present study is, to our best knowledge, the first to use a validated volume measuring tool, with a previous calculated criterion for significant growth, for analyzing radiological control rates after LINAC SRS or SRT for VS. A 4-year 
control rate of absence of additional intervention was found in $96.4 \%$ of the cases and radiological growth control rate was obtained in $85.4 \%$. In this series, transient swelling was defined as a decrease in volume to baseline volume. In one patient volume regressed to a volume above baseline volume. If this was considered as transient swelling, the radiological control rate would even improve.

In other studies, performing volume measurements after LINAC radiosurgery, radiological growth control was $84.4 \%$ and $81 \%$, respectively ${ }^{33,35}$. These control rates are influenced by the 'smallest detectable difference' (SDD), a measurement error, chosen by these authors, which was $13 \%$ and $20 \%$, respectively. Meijer et al. used a $13 \%$ SDD, referring to another study ${ }^{43}$ which calculated this percentage, although Meijer et al. used a different volume measuring technique. Okunaga et al. ${ }^{35}$ used $20 \%$ as measurement error, not mentioning on what basis this was chosen. A previous study showed that the measurement method used in the current study, has a measurement error of $19.7 \%{ }^{39}$.

A clear distinction between the "no additional intervention group" and "radiological control group" was seen. When analyzing the literature, this is an interesting observation: radiological growth control rates in 2D-studies range from $81 \%$ to $100 \%$ (median $96 \%$ ) 2, $, 4,7,-11,13-17,19-22,26-28,30,31,37,40$ and from $81 \%$ to $96.8 \%$ (median $90 \%$ ) in volume studies ${ }^{32,33,35-38}$. 2D-measurements are less reliable compared to volume measurements because a VS shows irregular growth in all directions, where 2D-studies usually examine only one or two dimensions. In addition, in 2D-studies, unreliable growth cut off points are used, that lie in measurement error ${ }^{39}$. This explains why postradiosurgical series using volume measurements generally report lower growth control rates compared to studies using 2D-measurements. "No additional intervention" rates are in keeping with other studies evaluating prognosis after radiosurgery in VS. They show a range of $91 \%$ to $100 \%$ (median $97 \%$ ) in 2D-studies ${ }^{2,5-7,9,10}$, $13,14,19,21,24,27,30,31,44$ and $95 \%$ to $100 \%$ (median $97 \%$ ) in volume studies ${ }^{32,35,37}$, both comparable to the rate of $96,4 \%$ obtained in this study. The discrepancy between the "radiological control group" and the "no additional intervention group" indicates that not all radiological growth is considerable enough for a patient to undergo a second VS related intervention.

\section{Growth patterns}

Three specific VS growth patterns could be identified after LINAC SRS or SRT (Figure 4.3). They resemble previously defined growth patterns after LINAC $\mathrm{SRS} / \mathrm{SRT}^{35}$, although now they are calculated with a validated measuring tool $^{39}$.

The occurrence of transient postradiosurgical swelling, which was often misunderstood as treatment failure in the early years of treating VS with 
radiosurgery, is interesting. It is a common phenomenon with an incidence ranging from $3 \%$ to $74 \%$, $7,17,21,28,33-38$. This range is caused by differences in defining transient swelling and measuring techniques. It is probably caused by peritumoral edema. In the present study, $54 \%$ of the patients appeared with transient VS swelling. Previous studies showed that transient swelling after radiosurgery occurred generally within the first year posttreatment ${ }^{7,17,21,28,34,35,37,38}$ and size usually regressed within 2 years ${ }^{7,17}$. This validated study, found maximum swelling after a median of 5 months (range 3-17) and first measured regression was seen after a median of 15 months (range 8-27). Volume of this temporary increased size decreased beyond baseline size after a median of 24 months (range 11-62). The time frame in which transient swelling may occur has been reported in one volumetrical LINAC study as well: Meijer et al. found this decrease after a median of 34 months (range 20-55) ${ }^{33}$, indicating that the occurrence of transient swelling is a phenomenon that can last long postradiosurgically.

Some authors tried to create time frames in order to differentiate transient swelling from continuous growth ${ }^{7,35}$. They conclude that a first FU MRI after 2 years is necessary in order to differentiate between transient swelling and continuous growth. Other authors conclude that the first FU measurement should not be performed before the first 20 months after LINAC radiosurgery, and the second MRI should be performed after 55 months in order to differentiate swelling from genuine progression ${ }^{33}$. In the ideal case FU should be performed at a point where one can differentiate between transient swelling and continuous growth in order to prevent patients from undergoing unnecessary interventions. This, however, seems impossible when taking the wide range of months after SRS or SRT into account during which transient swelling may last. Frequent FU with a one year interval, or, in absence of growth or clinical symptomatology, a two year interval, is essential in order to identify patients with growth in time and to avoid possible complications by intervening forehanded. Increase in volume in absence of symptomatology should be watched carefully, in order to prevent unnecessary interventions due to transient swelling.

\section{Prognostic factors}

Some studies suggest that the presence of central nonenhancement after SRS or SRT indicates good VS control ${ }^{32,45}$ or indicates VS shrinkage ${ }^{20,45}$. Central nonenhancement was seen in $59 \%$ of the patients after LINAC SRS or SRT, matching the wide range published elsewhere which ranges from 4-93\% $4,7,8,12$, $16,17,19-21,25,27,31,32,35,36,40$. In this study, central nonenhancement did not prove to be a reliable indicator regarding VS radiological growth control. Six patients with cystic components before treatment were identified. Other authors described worse outcome in patients with cystic VS after radiosurgery, because 
of possible rapid expansion of the cystic component ${ }^{46-48}$. This could not be reconfirmed in this study, eventually due to the small number of patients with cystic VS. Furthermore, no other factors were found predicting radiological failure. This is comparable to most results published previously, although Hasegawa et al., using Gamma knife surgery (GKS) and 2D-measurements for $\mathrm{FU}$, reports that the factors female gender, VS volume $>15 \mathrm{~cm}^{3}$ and patients undergoing previous treatment were associated significantly with VS radiological growth ${ }^{14}$. This was not found in the present study. The latter study also found sex and larger tumor volume to be associated with transient swelling. Another study, using volume measurements on VS after GKS, found a high dose treatment related to the occurrence of transient swelling ${ }^{34}$. The present study found the presence of previous treatment to be associated with the occurrence of transient swelling significantly, as was treating patients with SRS: a higher dose of radiation in one time clearly influences the postradiosurgical reaction of the VS. A good explanation for the occurrence of transient swelling in patients receiving prior treatment was not found. These factors may be taken into account when increase in volume is seen after SRS.

\section{Limitations}

This study also harbours some limitations. Firstly, median FU time (40,0 months) is relatively short, and longer FU may be necessary to draw definite conclusions. The second limitation lies in the usage of two different radiosurgical techniques (SRS/SRT). Thirdly, the lack of symptom-based clinical FU parameters limits the conclusions that can be drawn from the data.

\section{Conclusion}

In this study, in which a validated volume measuring tool was used, good control rates are reported for LINAC based SRS/SRT in VS, both in the "radiological control group" and the "no additional intervention group". The lower rate of radiological control is attributed to the use of the more sensitive volume measurements. Transient swelling after radiosurgery is a common phenomenon and should not be mistaken for treatment failure. Previous treatment and the use of SRS were associated with a higher risk of transient swelling. 


\section{References}

1. Stangerup SE, Caye-Thomasen $P$, Tos $M$, Thomsen J. The natural history of vestibular schwannoma. Otol Neurotol. 2006;27:547-52.

2. Kondziolka D, Lunsford LD, McLaughlin MR, Flickinger JC. Long-term outcomes after radiosurgery for acoustic neuromas. N Engl J Med. 1998;339:1426-33.

3. Pollock BE, Driscoll CL, Foote RL, Link MJ, Gorman DA, Bauch CD, Mandrekar $\mathrm{JN}$, Krecke $\mathrm{KN}$, Johnson $\mathrm{CH}$. Patient outcomes after vestibular schwannoma management: a prospective comparison of microsurgical resection and stereotactic radiosurgery. Neurosurgery. 2006;59:77-85; discussion 77-85.

4. Combs SE, Thilmann C, Debus J, Schulz-Ertner D. Long-term outcome of stereotactic radiosurgery (SRS) in patients with acoustic neuromas. Int J Radiat Oncol Biol Phys. 2006;64:1341-7.

5. Flickinger JC, Kondziolka D, Niranjan A, Lunsford LD. Results of acoustic neuroma radiosurgery: an analysis of 5 years' experience using current methods. J Neurosurg. 2001;94:1-6.

6. Hasegawa T, Kida Y, Yoshimoto M, Koike J, Goto K. Evaluation of tumor expansion after stereotactic radiosurgery in patients harboring vestibular schwannomas. Neurosurgery. 2006;58:1119-28; discussion -28.

7. Nakamura H, Jokura H, Takahashi K, Boku N, Akabane A, Yoshimoto T. Serial follow-up MR imaging after gamma knife radiosurgery for vestibular schwannoma. AJNR Am J Neuroradiol. 2000;21:1540-6.

8. Bertalanffly A, Dietrich W, Aichholzer M, Brix R, Ertl A, Heimberger K, Kitz K. Gamma Knife Radiosurgery for Acoustic Neurinomas. Acta Neurochir. 2001;143:689-95.

9. Chang SD, Gibbs IC, Sakamoto GT, Lee E, Oyelese A, Adler JR, Jr. Staged stereotactic irradiation for acoustic neuroma. Neurosurgery. 2005;56:1254-61; discussion 61-3.

10. Chopra R, Kondziolka D, Niranjan A, Lunsford LD, Flickinger JC. Long-term followup of acoustic schwannoma radiosurgery with marginal tumor doses of 12 to 13 Gy. Int J Radiat Oncol Biol Phys. 2007;68:845-51.

11. Combs SE, Volk S, Schulz-Ertner D, Huber PE, Thilmann C, Debus J. Management of acoustic neuromas with fractionated stereotactic radiotherapy (FSRT): long-term results in 106 patients treated in a single institution. Int J Radiat Oncol Biol Phys. 2005;63:75-81.

12. Delsanti C, Roche PH, Thomassin JM, Regis J. Morphological changes of vestibular schwannomas after radiosurgical treatment: pitfalls and diagnosis of failure. Prog Neurol Surg. 2008;21:93-7.

13. Godefroy WP, Bakker CV, Kaptein AA, Romero AM, Linge v, A, Nowak PJ, Dallenga $\mathrm{AH}$, Wolbers JG. Quality of life and clinical outcome after radiosurgery for vestibular schwannoma. Submitted for publication.

14. Hasegawa T, Fujitani S, Katsumata S, Kida Y, Yoshimoto M, Koike J. Stereotactic radiosurgery for vestibular schwannomas: analysis of 317 patients followed more than 5 years. Neurosurgery. 2005;57:257-65; discussion -65.

15. Iwai $\mathrm{Y}$, Yamanaka K, Shiotani M, Uyama T. Radiosurgery for acoustic neuromas: results of low-dose treatment. Neurosurgery. 2003;53:282-87; discussion 7-8.

16. Kalogeridi M-A, Georgolopoulou P, Kouloulias V, Kouvaris J, Pissakas G. Longterm results of LINAC-based stereotactic radiosurgery for acoustic neuroma: The Greek experience. J Can Res Ther. 2009;5:8-13. 
17. Kobayashi T, Tanaka T, Kida $\mathrm{Y}$. The early effects of gamma knife on 40 cases of acoustic neurinoma. Acta Neurochir Suppl. 1994;62:93-7.

18. Koh ES, Millar BA, Menard C, Michaels H, Heydarian M, Ladak S, McKinnon S, Rutka JA, Guha A, Pond GR, Laperriere NJ. Fractionated stereotactic radiotherapy for acoustic neuroma: single-institution experience at The Princess Margaret Hospital. Cancer. 2007;109:1203-10.

19. Kwon Y, Khang SK, Kim CJ, Lee DJ, Lee JK, Kwun BD. Radiologic and histopathologic changes after Gamma Knife radiosurgery for acoustic schwannoma. Stereotact Funct Neurosurg. 1999;72 Suppl 1:2-10.

20. Linskey ME, Lunsford LD, Flickinger JC. Neuroimaging of acoustic nerve sheath tumors after stereotaxic radiosurgery. AJNR Am J Neuroradiol. 1991;12:1165-75.

21. Lunsford LD, Niranjan A, Flickinger JC, Maitz A, Kondziolka D. Radiosurgery of vestibular schwannomas: summary of experience in 829 cases. J Neurosurg. 2005;102 Suppl:195-9.

22. McClelland S, 3rd, Gerbi BJ, Higgins PD, Orner JB, Hall WA. Safety and efficacy of fractionated stereotactic radiotherapy for acoustic neuromas. J Neurooncol. 2008;86:191-4.

23. Meijer OW, Vandertop WP, Baayen JC, Slotman BJ. Single-fraction vs. fractionated linac-based stereotactic radiosurgery for vestibular schwannoma: a single-institution study. Int J Radiat Oncol Biol Phys. 2003;56:1390-6.

24. Miller RC, Foote RL, Coffey RJ, Sargent DJ, Gorman DA, Schomberg PJ, Kline RW. Decrease in cranial nerve complications after radiosurgery for acoustic neuromas: a prospective study of dose and volume. Int J Radiat Oncol Biol Phys. 1999;43:305-11.

25. Pollock BE. Management of vestibular schwannomas that enlarge after stereotactic radiosurgery: treatment recommendations based on a 15 year experience. Neurosurgery. 2006;58:241-8; discussion -8.

26. Rutten I, Baumert BG, Seidel L, Kotolenko S, Collignon J, Kaschten B, Albert A, Martin D, Deneufbourg JM, Demanez JP, Stevenaert A. Long-term follow-up reveals low toxicity of radiosurgery for vestibular schwannoma. Radiother Oncol. 2007;82:83-9.

27. Shirato H, Sakamoto T, Takeichi N, Aoyama H, Suzuki K, Kagei K, Nishioka T, Fukuda S, Sawamura Y, Miyasaka K. Fractionated stereotactic radiotherapy for vestibular schwannoma (VS): comparison between cystic-type and solid-type VS. Int J Radiat Oncol Biol Phys. 2000;48:1395-401.

28. Spiegelmann R, Lidar Z, Gofman J, Alezra D, Hadani M, Pfeffer R. Linear accelerator radiosurgery for vestibular schwannoma. J Neurosurg. 2001;94:7-13.

29. Williams JA. Fractionated stereotactic radiotherapy for acoustic neuromas: preservation of function versus size. J Clin Neurosci. 2003;10:48-52.

30. Friedman WA, Bradshaw P, Myers A, Bova FJ. Linear accelerator radiosurgery for vestibular schwannomas. J Neurosurg. 2006;105:657-61.

31. Chan AW, Black PM, Ojemann RG, Barker FG, Kooy HM, Lopes VV, . Stereotactic Radiotherapy for Vestibular Schwannomas: Favorable Outcome with Minimal Toxicity. Neurosurgery. 2005;57:60-70.

32. Chung WY, Liu KD, Shiau CY, Wu HM, Wang LW, Guo WY, Ho DM, Pan DH. Gamma knife surgery for vestibular schwannoma: 10-year experience of 195 cases. J Neurosurg. 2005;102 Suppl:87-96.

33. Meijer OW, Weijmans EJ, Knol DL, Slotman BJ, Barkhof F, Vandertop WP, Castelijns JA. Tumor-volume changes after radiosurgery for vestibular 
schwannoma: implications for follow-up MR imaging protocol. AJNR Am J Neuroradiol. 2008;29:906-10.

34. Nagano O, Higuchi Y, Serizawa T, Ono J, Matsuda S, Yamakami I, Saeki N. Transient expansion of vestibular schwannoma following stereotactic radiosurgery. J Neurosurg. 2008;109:811-6.

35. Okunaga T, Matsuo T, Hayashi N, Hayashi Y, Shabani HK, Kaminogo M, Ochi M, Nagata I. Linear accelerator radiosurgery for vestibular schwannoma: measuring tumor volume changes on serial three-dimensional spoiled gradient-echo magnetic resonance images. J Neurosurg. 2005;103:53-8.

36. Prasad D, Steiner M, Steiner L. Gamma surgery for vestibular schwannoma. J Neurosurg. 2000;92:745-59.

37. Wowra B, Muacevic A, Jess-Hempen A, Hempel JM, Muller-Schunk S, Tonn JC. Outpatient gamma knife surgery for vestibular schwannoma: definition of the therapeutic profile based on a 10-year experience. J Neurosurg. 2005;102 Suppl:114-8.

38. Yu CP, Cheung JY, Leung S, Ho R. Sequential volume mapping for confirmation of negative growth in vestibular schwannomas treated by gamma knife radiosurgery. J Neurosurg. 2000;93 Suppl 3:82-9.

39. van de Langenberg R, de Bondt BJ, Nelemans PJ, Baumert BG, Stokroos RJ. Follow-up assessment of vestibular schwannomas: volume quantification versus two-dimensional measurements. Neuroradiology. 2009.

40. Flickinger JC, Lunsford LD, Coffey RJ, Linskey ME, Bissonette DJ, Maitz AH, Kondziolka D. Radiosurgery of acoustic neurinomas. Cancer. 1991;67:345-53.

41. Bethesda MD. ICRU Report 50. Prescribing, recording, and reporting photon beam therapy. International Commission on Radiation Units and Measurements 20814 USA. 1993.

42. Baumert BG, Villà S, Studger G, Mirimanoff R-O, Davis JB, Landau K. Early improvements in vision after fractionated stereotactic radiotherapy for primary optic nerve sheath meningioma. Radiotherapy and Oncology. 2004;72:169-74.

43. Vokurka EA, Herwadkar A, Thacker NA, Ramsden RT, Jackson A. Using Bayesian tissue classification to improve the accuracy of vestibular schwannoma volume and growth measurement. AJNR Am J Neuroradiol. 2002;23:459-67.

44. Roos DE, Brophy BP, Bhat MK, Katsilis ES. Update of radiosurgery at the Royal Adelaide Hospital. Australas Radiol. 2006;50:158-67.

45. Lunsford LD, Kondziolka D, Maitz A, Flickinger JC. Black holes, white dwarfs and supernovas: imaging after radiosurgery. Stereotact Funct Neurosurg. 1998;70 Suppl 1:2-10.

46. Charabi S, Klinken L, Tos M, Thomsen J. Histopathology and growth pattern of cystic acoustic neuromas. Laryngoscope. 1994;104:1348-52.

47. Pendl G, Ganz JC, Kitz K, Eustacchio S. Acoustic neurinomas with macrocysts treated with Gamma Knife radiosurgery. Stereotact Funct Neurosurg. 1996;66 Suppl 1:103-11.

48. Wandong S, Meng L, Xingang L, Yuguang L, Shugan Z, Lei W, Chengyuan W. Cystic acoustic neuroma. J Clin Neurosci. 2005;12:253-5. 


\section{MANAGEMENT OF LARGE VESTIBULAR SCHWANNOMA. PART I. PLANNED SUBTOTAL RESECTION FOLLOWED BY GAMMA KNIFE SURGERY: RADIOLOGICAL AND CLINICAL ASPECTS}

Rick van de Langenberg

Patrick EJ Hanssens Jacobus J van Overbeeke Jeroen B Verheul Patty J Nelemans Bert J de Bondt Robert J Stokroos

Journal of Neurosurgery. 2011;115:875-84 


\section{Abstract}

\section{Object}

In large vestibular schwannoma (VS), microsurgery is the main treatment option, and complete resection is considered the primary goal. However, previous studies have documented suboptimal facial nerve outcomes in patients who underwent complete resection of large VSs. Subtotal resection is likely to reduce the risk of facial nerve injury but increases the risk of lesion regrowth. Gamma Knife surgery (GKS) can be performed to achieve long-term growth control of residual VS after incomplete resection. In this study the authors report on the results in patients treated by using planned subtotal resection followed by GKS with special attention to volumetric growth, control rate and symptoms.

\section{Methods}

Fifty consecutive patients who underwent the combined treatment strategy of subtotal microsurgical resection and GKS for large VSs between 2002 and 2009 were retrospectively analyzed. Patients with neurofibromatosis Type 2 were excluded. Patient charts were reviewed for clinical symptoms. Audiograms were evaluated in order to classify hearing pre- and postoperatively. Pre-operative and follow-up contrast enhanced T1-weighted MR images were analyzed using volume-measuring software.

\section{Results}

Surgery was performed via a translabyrinthine (25 patients) or retrosigmoid (25 patients) approach. The median follow-up was 33.8 months. Clinical control was achieved in $92 \%$ of the cases and radiological control in $90 \%$. One year after radiosurgery facial nerve function was good (House Brackmann Grade I or II) in $94 \%$ of the patients. One of the two patients who underwent surgery to preserve hearing maintained serviceable hearing after resection followed by GKS.

\section{Conclusion}

Considering the good growth control and facial nerve function preservation as well as the possibility of preserving serviceable hearing and the low number of complications, a subtotal resection followed by GKS can be the treatment option of choice for large VSs 


\section{Introduction}

Vestibular schwannomas (VS) are benign neoplasms originating from the vestibular part of the cranial nerve VIII. Their incidence is increasing, mostly because of the aging population and increased use of and improvements in MR imaging ${ }^{1,2}$. Three main treatment modalities exist: radiosurgery, microsurgery or a so-called wait and scan policy, in which patients' audiovestibular symptoms are monitored regularly combined with sequential MR imaging to follow VS growth ${ }^{3}$. When dealing with large VSs, a treatment dilemma arises: a wait and scan policy is not recommended given the possibility of lifethreatening complications due to further VS progression. The complications of radiosurgery are related to dose and volume and the procedure can cause (transient) VS swelling ${ }^{4,5}$, which is one reason that this therapy is generally avoided in large VSs. With the complete microsurgical removal of large VSs, a considerable risk of long-term suboptimal cranial nerve outcome arises and good facial nerve function (House Brackmann (HB) Grade I or II) is reported in only $27-58 \%$ of the patients ${ }^{6-12}$. Controversy surrounds complete removal for tumor control versus subtotal removal for preservation of facial nerve function in large VSs. If removed subtotally, these lesions do not usually stop growing ${ }^{13-23}$. Previous studies have revealed excellent long-term growth control with minimal risk to the cranial nerves after GKS for primary and residual $V_{S s}{ }^{21,24-29}$. Therefore, in the management of large VSs, the combination of subtotal VS resection and GKS might be a logical treatment strategy to decrease the risk of both nerve damage and growth of residual VS. The authors of only four studies have reported outcomes after subtotal resection followed by GKS in large VSs ${ }^{20,30-32}$, although the number of included patients was small ${ }^{20,30,31}$ and only two dimensional (2D) measurements were performed, which are less reliable than volume measurements to evaluate tumor control ${ }^{33}$. The present study was conducted to evaluate the strategy of subtotal removal combined with GKS for large VSs, with special attention to their clinical and radiological control, facial nerve preservation and hearing function.

\section{Methods}

\section{Patient population}

Patients who underwent planned subtotal VS resection with postoperative GKS between January 1, 2002 and January 1, 2009 at the GammaKnife Center, Tilburg were analyzed retrospectively. The diagnosis of VS was confirmed histopathologically in all cases. The indication for surgery was a large VS, indenting the brain stem, according to the Koos classification system ${ }^{34}$. Patients with Koos Grade III lesions underwent surgery after growth was seen 
after one year of follow-up, whereas an intervention without further follow-up was planned in patients with Koos Grade IV lesions (Figure 5.1a). The possibility of primary GKS was discussed preoperatively with patients harboring a VS smaller than $4 \mathrm{~cm}$ in the extracanalicular dimension and without symptomatic mass effect. They were informed about the diminished reserve for seeing VS growth or transient swelling after primary radiosurgery, possibly resulting in a higher chance of treatment failure. Vestibular schwannomas $4 \mathrm{~cm}$ or larger were considered to be poor candidates for primary GKS because some degree of symptomatic mass effect is usually present. The results of primary GKS for large VSs are published separately ${ }^{35}$.

A surgical team consisting of a neurotologist and a neurosurgeon conducted most surgical procedures. Surgery was performed via either a translabyrinthine or a retrosigmoid approach, depending on surgeon preference, tumor size and auditory function of the involved ear. The primary goal of surgery was brainstem decompression, and the facial nerve was identified using neurostimulation. The operating neurosurgeon was involved in the postoperative radiosurgical planning. Generally, microsurgery was continued until adequate brain stem decompression was achieved, to a volume well suitable for GKS. The contour of the VS remnant was evaluated on the basis of the neuronavigation. The operating team never continued to a full VS resection, no internal auditory canal decompression was performed when operating retrosigmoidally. Postoperatively, all patients received GKS.

\section{Surgical technique}

Stereotactic radiosurgery was performed using a Leksell Gamma Knife 4C (Elekta $A B$ ). Treatment planning was made with Leksell GammaPlan (version 8.3.1). A dose of 12.0 to $13.0 \mathrm{~Gy}$ (mean, $12.9 \mathrm{~Gy}$ ) was prescribed to the isodose covering $90 \%$ of the tumor volume. The maximum dose varied from 18.0 to 26.0 Gy (mean, $21.1 \mathrm{~Gy}$ ). The tumor margin dose varied from 9.4 to $11.9 \mathrm{~Gy}$ (mean $11.0 \mathrm{~Gy}$ ). The number of isocenters per patient varied from 7 to 44 (mean 22.8 isocenters).

Clinical and radiological follow-up was planned for 3 months, 1 year and 2 years after GKS and then once every 2 years thereafter if the tumor size shrank or was stable. A retrospective chart review was performed to evaluate clinical symptoms at presentation, after microsurgery and GKS. Audiometric assessments were performed in accordance with the American Academy of Otolaryngology-Head and Neck Surgery guidelines, consisting of a mean pure tone average of $0.5,1,2$ and $4 \mathrm{kHz}$ and speech discrimination score (\%). According to these results, patients were classified in hearing classes $A, B, C$ or $D$; where $A$ and $B$ are considered as serviceable hearing and $C$ and $D$ are not $^{36}$. Facial nerve function was scored according to the HB scale ${ }^{37}$ at the first 
visit to the outpatient department postoperatively and 1 year after GKS. Good postoperative facial nerve function was defined as HB Grades I and II.

One reader experienced in VS imaging performed all radiological examinations of axial Gd-enhanced $(0.1 \mathrm{mg} / \mathrm{kg}$ body weight) T1-weighted MR images. The slice thickness was usually 1 or 2 millimeter, without slice gap. Volumetric measurements were performed preoperatively, on the planning MR imaging and after GKS. These volumes were calculated using Leksell GammaPlan 8.3.1 software. Central nonenhancement was defined as a hypo-intense area in the center of VS on contrast enhanced T1-weighted MR imaging not present before treatment, and scored as either absent or present. Cystic VSs were defined as VSs with enhanced cysts making up at least one-third of nonenhancement of the tumor volume on contrast enhanced T1-weighted images and enhancing on T2-weighted images.

Radiological control was defined as VS regression or the absence of significant growth beyond measurement error, which has been defined as a difference of $19.7 \%$ between subsequent scans ${ }^{33}$. Clinical VS control was defined as the absence of the need for further treatment (GKS or microsurgery) after subtotal VS resection and planned GKS.

\section{Statistical analysis}

Absolute changes in volume were calculated by subtracting the VS volume at the time of GKS from its volume at the last measurement (volume at $t_{x}$-volume at $t_{0}$ ). Relative changes in volume were calculated as absolute change divided by the VS volume at the time of GKS $\left(\mathrm{t}_{0}\right)$ : (volume at $\mathrm{t}_{\mathrm{x}}$-volume at $\left.\mathrm{t}_{0}\right)$ / volume at $t_{0}$. This relative change was multiplied by 100 to obtain a percentage change in volume. A similar algorithm was used to determine the amount of residual VS tissue after surgery. The cumulative probability of clinical and radiological control was calculated using Kaplan-Meier analyses. Differences in mean values were tested for significance using the independent samples $t$-test. Fisher exact and $\chi^{2}$ test were used for categorical data. For all tests, P-values $\leq 0.05$ were considered to indicate statistical significance. All analyses were performed using SPSS 15.0 statistical software (SPSS, Inc.).

\section{Results}

\section{Patient and intervention characteristics}

There were 28 males and 22 females, with a mean age of 52.0 years (range 21-84 years), included in this study. All patients reported hearing loss on presentation and only $4(8 \%)$ of the 50 patients presented with serviceable hearing, all Class B. Twenty-four (48\%) of the 50 patients presented with 
coexisting vertigo, 19 (38\%) with tinnitus and 10 (20\%) with ataxia. Furthermore, $14(28 \%)$ appeared with facial hypesthesia and $1(2 \%)$ with facial weakness. Two patients presented with symptomatic hydrocephalus, for which ventriculoperitoneal (1 patient) and lumbar (1 patient) drainage was necessary. The mean VS size was $14.9 \mathrm{~cm}^{3}$ (range $4.3-36.1 \mathrm{~cm}^{3}$ ). Further clinical and radiological features at presentation are presented in Tables 5.1 and 5.2. Twenty-five patients (50\%) underwent surgery via a retrosigmoid approach and $25(50 \%)$ via a translabyrinthine approach. Mean time between microsurgery and GKS was 8.5 months (range 2-24 months). All patients underwent a subtotal resection. Mean postoperative VS volume on the first follow-up MR image was $3.34 \mathrm{~cm}^{3}$ (range $0.22-11.8 \mathrm{~cm}^{3}$ ), for a mean volume reduction of $74.6 \%$ (range $28-95 \%$ ) (Figure 5.1B and $5.1 \mathrm{C}$ ).

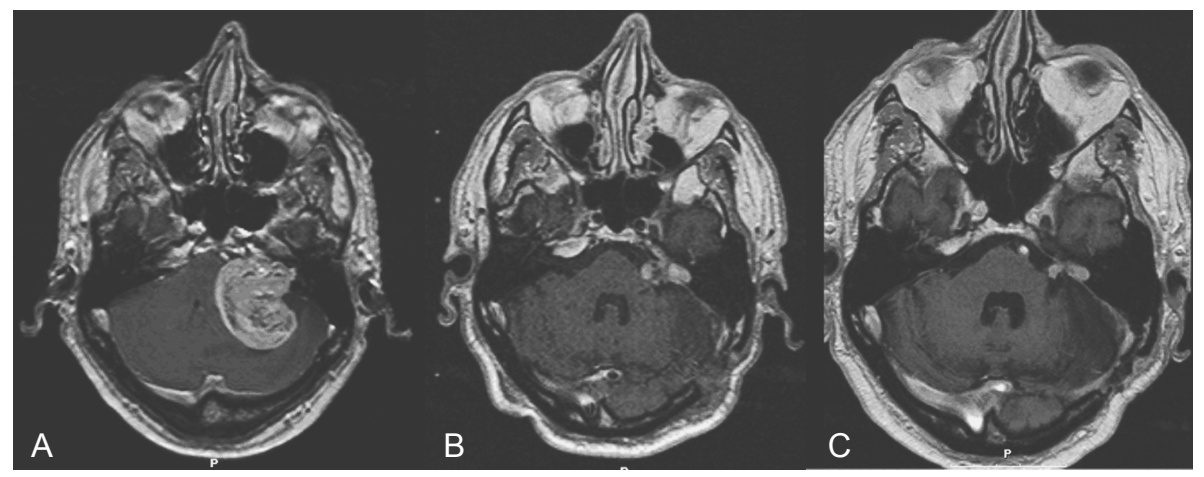

Figure 5.1 Axial contrast enhanced T1-weighted MR images obtained in a 68-year-old patient. Pre-operative image (A) showing a $35.9 \mathrm{~cm}^{3}$ left-sided vestibular schwannoma (Koos Grade IV). The patient underwent a retrosigmoidal subtotal resection, resulting in a $3.9 \mathrm{~cm}^{3}$ remnant (B). Four months postoperatively Gamma Knife surgery was performed. During the 4 years of follow up the remnant remained stable, and the patient is in good clinical condition, with facial nerve function scored as House Brackmann Grade II (C).

\section{Clinical and radiological control}

Clinical and radiological VS control was achieved in $92 \%$ and $90 \%$ of cases, respectively. The median follow-up was 33.8 months (range 12-84 months). The cumulative probability of clinical and radiological control is shown in the Kaplan-Meier analyses (Figure 5.2 and 5.3). One patient died of a cardiac arrest, 5 years after GKS. No patient- or VS-related characteristic (age, sex, lesion location, pre- or postoperative lesion size, central nonenhancement, presence of cystic components, surgical approach and time between microsurgery and GKS) was predictive of clinical or radiological failure or worse facial nerve outcome ( $P=$ not significant). Clinical treatment failure occurred after a mean of 31.5 months (range $22-49$ months). 
Table 5.1 Summary of characteristics in 50 patients before microsurgery for vestibular schwannoma

\begin{tabular}{|c|c|}
\hline Characteristic & No. \\
\hline Men/Women & $28(56) / 22(44)$ \\
\hline Mean age in yrs (range) & $52.0(21-84)$ \\
\hline Median FU in mos (range) & $33.8(12-84)$ \\
\hline Mean time between operation and GKS in mos (range) & $8.5(2-24)$ \\
\hline \multicolumn{2}{|l|}{ Symptom (\%) } \\
\hline Tinnitus & $19(38)$ \\
\hline Vertigo & $24(48)$ \\
\hline Trigeminal hypesthesia & $14(28)$ \\
\hline Ataxia & $10(20)$ \\
\hline Facial weakness & $1(2)$ \\
\hline Symptomatic hydrocephalus necessitating drainage & $2(4)$ \\
\hline Ventriculoperitoneal drainage & $1(2)$ \\
\hline Lumbar drainage & $1(2)$ \\
\hline Papiledema & $1(2)$ \\
\hline \multicolumn{2}{|l|}{ Hearing class (\%) } \\
\hline A & $0(0)$ \\
\hline B & $4(8)$ \\
\hline $\mathrm{C}$ & $10(20)$ \\
\hline D & $36(72)$ \\
\hline \multicolumn{2}{|l|}{ VS side $(\%)$} \\
\hline Left & $24(48)$ \\
\hline Right & $26(52)$ \\
\hline Cystic VS (\%) & $17(34.7)$ \\
\hline Mean VS vol in $\mathrm{cm}^{3}$ (range) & $14.9(4.1-36.1)$ \\
\hline Mean VS diameter in cm (range) & $3.5(2.6-5.4)$ \\
\hline \multicolumn{2}{|l|}{ Extracanalicular dimension in $\mathrm{cm}$} \\
\hline $2-3$ & 8 \\
\hline $3-4$ & 23 \\
\hline 4-5 & 16 \\
\hline$>5$ & 3 \\
\hline Koos Grade & 7 \\
\hline $\mathrm{I}-\mathrm{II}$ & 0 \\
\hline III & 7 \\
\hline IV & 43 \\
\hline \multicolumn{2}{|l|}{ Hannover classification } \\
\hline $\mathrm{T} 1-3 \mathrm{~b}$ & 0 \\
\hline T4a & 7 \\
\hline $\mathrm{T} 4 \mathrm{~b}$ & 43 \\
\hline
\end{tabular}

GKS= Gamma Knife surgery. Mos = months. Hearing classes according to the classification of the American Academy of Otolaryngology- Head and Neck Surgery, where classes A and B are considered as serviceable hearing and $C$ and $D$ not. Koos gradation: Grade I: intracanalicular VS, Grade II: extending into the cerebellopontine angle, Grade III: compressing the brain stem, Grade IV: deviating the $4^{\text {th }}$ ventricle. Hannover classification: T Class T1, intracanalicular VS; Class T2, intra- and extracanalicular tumor; Class T3a, filling the cerebellopontine cistern; Class T3b, VS reaching the brainstem; Class T4a, VS compressing the brainstem; and Class T4b, VS severely dislocating the brainstem and compressing the $4^{\text {th }}$ ventricle. 
Table 5.2 Surgical procedures and control rates.

$\begin{array}{lc}\text { Surgical Procedure } & \text { No. }(\%) \\ \text { Retrosigmoidal } & 25(50) \\ \text { Translabyrinthine } & 25(50) \\ \text { Control rates } & \\ \quad \text { Clinical control rate } & 46(92) \\ \text { Second GKS } & 3(6) \\ \text { Second MS followed by GKS } & 1(2) \\ \text { Radiological control rate } & 45(90)\end{array}$

GKS= Gamma Knife surgery. MS= Microsurgery.

Table 5.3 Characteristics of patients experiencing clinical failure.

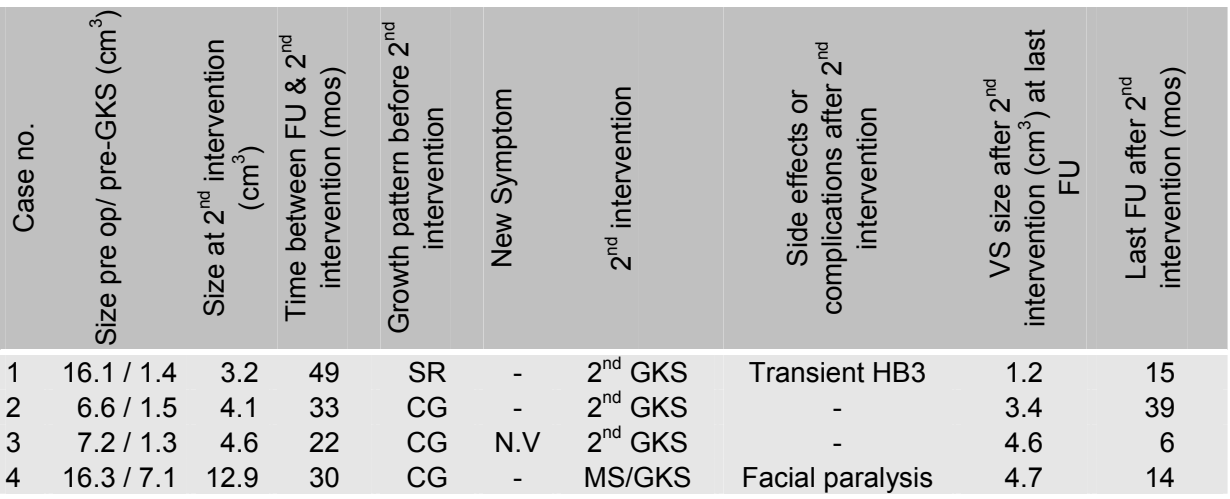

$\mathrm{CG}=$ continuous growth. $\mathrm{FU}=$ follow-up. $\mathrm{GKS}=$ Gamma Knife surgery. $\mathrm{HB}=$ House Brackmann grade for facial nerve function. MS=microsurgery. NV=trigeminal hypesthesia. SR=regrowth after shrinkage.

Of the 4 patients whose lesion showed significant growth, 3 underwent a second GKS treatment for residual VS, and thereafter, stability or a significant reduction of volume occurred during a median follow-up of 14.5 months (Table 5.3). After the second GKS, 1 patient suffered from an HB Grade 3 transient facial nerve paresis. The fourth patient underwent a second microsurgical excision followed by GKS 49 months after the first resection because of progressing regrowth on MR imaging despite the absence of clinical symptoms. This case involved a large VS remnant. Despite facial nerve monitoring and microsurgical precision, no facial nerve response was noted at the end of the operation due to a facial nerve paralysis. A hypoglossal-facial nerve anastomosis was performed. One patient appearing with significant lesion growth still undergoes a wait and scan policy, 22 months after first GKS. 


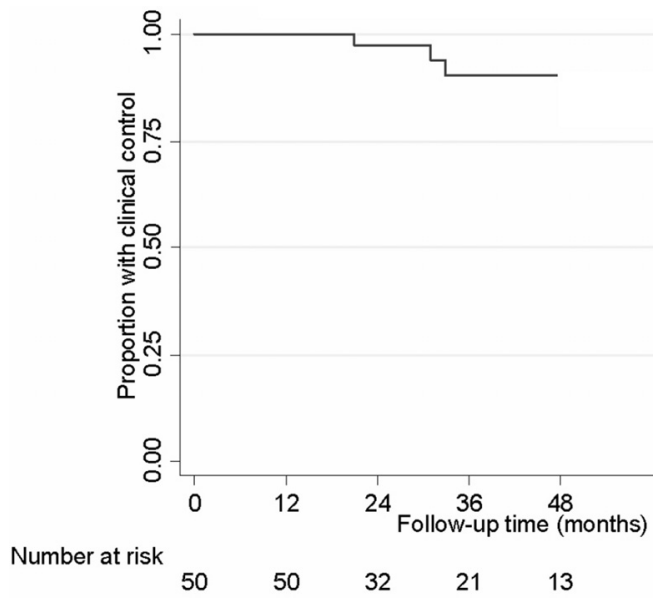

Figure 5.2 Kaplan-Meier analysis of clinical control during follow-up after subtotal resection followed by Gamma Knife surgery for large vestibular schwannomas.

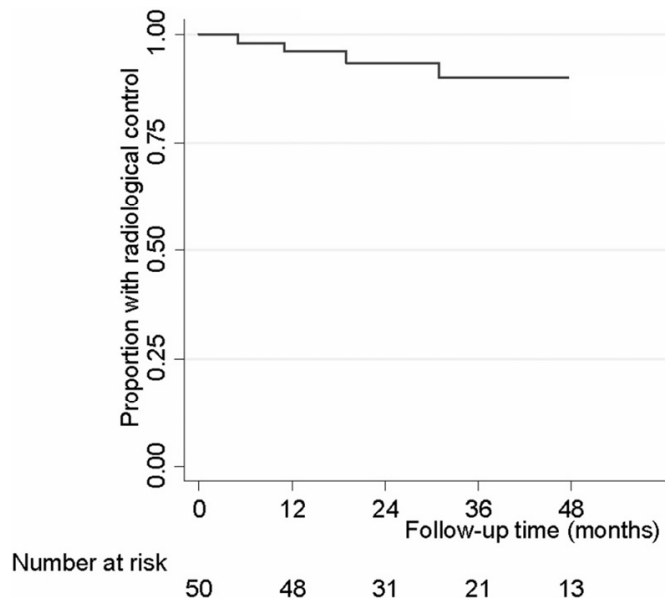

Figure 5.3 Kaplan-Meier analysis of radiological control during follow-up after subtotal resection followed by Gamma Knife surgery for large vestibular schwannomas.

\section{Cranial nerve preservation}

Good facial nerve function (HB Grades I and II) was preserved after microsurgery and GKS in $94 \%$ of the patients (Table 5.4). Three patients ended up with an HB Grade higher than II: one patient underwent a hypoglossal-facial nerve anastomosis because of a total facial paralysis. One patient underwent a lateral tarsorrhaphy and gold implantation because of an HB Grade IV facial 
nerve paresis, and one patient received mime-therapy because of HB Grade III function. The first patient had a large VS (extracanalicular dimension $>5 \mathrm{~cm}$ ) where intraoperatively significant venous bleeding occurred. Despite surgical precision, facial nerve stimulation weakened intraoperatively. The second patient suffered from a large compartimented cystic VS adherent to the cerebellum and brain stem. In third patient, for an unknown reason, facial nerve stimulation weakened when the upper part of the tumor was dissected, and thus surgery was stopped. At this point a broad decompression had been performed. Postoperatively, a total paralysis was seen, although it improved to HB Grade III function after 1 year. In the 4 patients presenting with serviceable hearing, 2 patients underwent a retrosigmoid approach to attempt hearing preserving. At the last follow-up (37 months), 1 of the 2 patients retained functional hearing.

Table 5.4 Distribution of facial nerve and cochlear function in 50 patients undergoing subtotal surgical resection of large vestibular schwannoma and planned Gamma Knife surgery.

$\begin{array}{lcc} & \begin{array}{c}\text { No }(\%) \\ \text { Fo }(\%)\end{array} \\ \text { House Brackmann Grade } & \text { One year after GKS } \\ \text { I } & 30(60) & \\ \text { II } & 4(8) & 41(82) \\ \text { III } & 7(14) & 6(12) \\ \text { IV } & 2(4) & 1(2) \\ \text { V } & 1(2) & 1(2) \\ \text { VI } & 6(12) & 0(0) \\ \text { Hearing scale } & & 1(2) \\ \text { A } & 0(0) & \text { Last follow-up } \\ \text { B } & 1(2)^{*} & 0(0) \\ \text { C } & 0(0) & 1(2) \\ \text { D } & 49(98) & 0(0) \\ \end{array}$

Facial nerve function is graded according to the House Brackmann facial nerve grading system and cochlear function according to AAO-HNS guidelines. * in only 2/4 patients with serviceable hearing a retrosigmoid approach was performed. GKS: Gamma Knife surgery.

\section{Patient complications}

One major complication of hematoma formation occurred in a patient who presented postoperatively due to a coagulation disorder that had not been discovered preoperatively; 2 revision surgeries were necessary to treat the hematoma. A permanent hemiparesis developed in this patient. Complications are presented in Table 5.5. After GKS, an HB Grade II transient facial paresis developed in 2 patients. One patient developed transient trigeminal hypesthesia. One patient developed persisting facial nerve spasms for which botulinum toxin injections have been administered every three months, with good results. 
Table 5.5 Minor and major complications after subtotal resection for a large vestibular schwannoma.

$\begin{array}{llc}\text { Complications } & \text { No. }(\%) \\ \text { Minor } & \text { Hematoma } & 2(4) \\ & \text { Wound infection } & 0(0) \\ & \text { CSF leakage } & 0(0) \\ & \text { Meningitis } & 0(0) \\ & \text { Hydrocephalus } & 2(4) \\ & \text { Ventriculoperitoneal drain } & 1(2) \\ & \text { Lumbar drain } & 1(2) \\ & \text { Temporary dysfunction CN IX, X } & 3(6) \\ & \text { Persisting dysfunction CN IX, X for which PEG placement } & 1(2) \\ & \text { Temporary abducens paresis } & 1(2) \\ \text { Fajor } & \\ & \text { Fecial and cochlear nerve: presented in table 5.4 } & 1(2) \\ & \text { Demiparesis } & 0(0)\end{array}$

$\mathrm{CN}=$ cranial nerve, $\mathrm{CSF}=$ cerebrospinal fluid. $\mathrm{PEG}=$ percutaneous endoscopic gastrostomy.

\section{Postradiosurgical growth patterns}

After GKS, 3 residual VS growth patterns were identified: 1) shrinkage, which was subdivided into direct shrinkage (23 [46\%] of 50 patients) and transient swelling followed by shrinkage (6 [12\%] of 50 patients); 2 ) stability, (16 [32\%] of 50 patients); and 3) growth, which was subdivided in continuous growth (4 [8\%] of 50 patients) and shrinkage followed by regrowth (1 [2\%] of 50 patients). Transient swelling occurred after a mean of 13.5 months after GKS (range 3-30 months). Twenty-five residual VSs $(50 \%)$ showed central nonenhancement after GKS. Three $(60 \%)$ of the 5 patients with lesion growth showed central nonenhancement, as compared with $22(96 \%)$ of 23 patients without lesion growth; however this difference was not significant $(P=0.70)$.

\section{Discussion}

The incidence of VS is increasing, given the aging of the population and the increased use and improvements in MR imaging ${ }^{1,2}$. More patients are being treated conservatively in a wait and scan policy ${ }^{38}$, in which audiovestibular symptoms are regularly monitored and VS size is observed with sequential MR imaging. The principle for this treatment is that some VSs do not grow or do so very slowly, and intervention may not be necessary ${ }^{39}$. However, large VSs may cause life-threatening symptomatology and a conservative approach is generally not recommended in these cases. Moreover, radiosurgery is rarely advised, because of the risk of transient VS swelling, which can lead to increased brain stem compression and concomitant symptoms. Microsurgery is considered the treatment of choice in these cases. The general consensus in 
VS surgery is to perform complete tumor removal. But doing so carries a considerable risk of facial nerve damage ${ }^{6-12}$ which in turn severely affects the quality of life ${ }^{40,41}$. These factors inform the rationale for the combined treatment of large VSs in the present study. Vestibular schwannoma volume is reduced by planned subtotal microsurgical removal in order to avoid possible neurologic deficits. Long-term growth control is achieved using planned GKS for residual VS. In addition, in case with serviceable hearing, a better chance of hearing preservation might be realized after this combined treatment as compared with the results following complete resection of a large $\mathrm{VS}^{7,8,42-45}$.

\section{Growth control}

In large VSs, complete lesion removal is advised ${ }^{9,46,47}$ to prevent the growth of residual VS. After long-term follow-up, however, recurrences have been described in $7 \%$ to $11 \%$ of the cases following complete VS removal ${ }^{20,21}$. This recurrence rate might be attributable to the fact that the anatomically intact facial nerves harbor schwannoma cells after surgery, as shown histopathologically ${ }^{48}$. In case of a subtotal resection, VS regrowth can occur up to $53 \%$ of patients ${ }^{13-17,19,21-23}$ and the chance of regrowth is considerably greater with increasing volumes of the residual $\mathrm{VS}^{13}$. Lownie et al. reported $18 \%$ regrowth after subtotal resection of large VSs ${ }^{18}$. Park et al. reported $11 \%$ regrowth after gross total resection and $32 \%$ regrowth after subtotal resection of large $\mathrm{VSs}^{20}$. Data in these studies indicate that the regrowth of subtotally resected VSs is an important issue, both for small/medium and for large VSs. Results after primary GKS in small/medium VSs are good, with growth control being documented between $87 \%$ to $97 \%$ of patients ${ }^{21,24-29}$. Studies evaluating GKS in large VSs provide growth control varying from $54 \%$ to $100 \%$, although drawing conclusions from these results is difficult because of the small number of patients included in these studies ${ }^{29,49-54}$. Results of the present study indicate that both clinical and radiological control can be achieved with GKS after subtotal resection of large VSs. In our therapeutic regimen, GKS is not deferred until growth is seen after surgery, because in the past large VSs have demonstrated the ability to grow and may do so after surgery. Ninety-two percent of the patients did not undergo a second intervention and $90 \%$ of the patients did not show significant lesion growth (Kaplan-Meier analysis, Figure 5.3). These rates are superior to those in the literature on the use of a subtotal resection alone $e^{13-17,19,21-23}$ and thus justify the use of GKS postoperatively.

Little is published regarding growth control in planned subtotal VS resection and GKS in large VS (Table 5.6). Fuentes et al. ${ }^{30}$ reported on 8 patients and achieved lesion growth control in $100 \%$ of them with a mean follow-up of 46 months. Iwai et al. ${ }^{31}$ studied 14 patients, including 2 with neurofibromatosis Type 2, and reported growth control in $79 \%$ during a mean follow-up of 32 months. Park et al. ${ }^{20}$ described 8 patients who underwent subtotal resection 
and GKS, with a $100 \%$ control rate with a mean follow-up of 68.8 months. The largest study to date is from Yang et al. ${ }^{32}$. They described a series of 61 patients, with a median follow-up of 53.7 months. An 8-year control rate of $93.5 \%$ was presented, although some of their patients received GKS more than 7 years after surgery; it was not mentioned whether this late GKS was due to VS growth. All previously published studies on the subtotal resection in large VSs followed by GKS were performed with 2D measurements, which are less reliable than volume measurements to assess growth control ${ }^{33}$.

The present study is the first to describe volumetric growth patterns in patients undergoing subtotal resection and planned GKS for large VSs. Three main radiological growth patterns were identified: shrinkage (58\%), stability (32\%) and growth (10\%). Shrinkage was subdivided into direct shrinkage (46\%) and transient swelling followed by shrinkage $(12 \%)$. Growth consisted of continuous growth $(8 \%)$ and shrinkage followed by regrowth $(2 \%)$. Yang et al. ${ }^{32}$ found radiological control in $94 \%$ of their cases, as compared with $90 \%$ in the present study. Note, however, that a direct comparison of the results is hampered by differences in the technique of volume measurements, that is, $2 \mathrm{D}$ versus volume measurements. Furthermore, differences in patient selection, radiosurgical technique and duration of follow-up make direct comparison of the results troublesome, although the diminished accuracy in detecting growth by using $2 \mathrm{D}$ measurements might be the reason for the higher radiological growth control.

The growth patterns for subtotally resected VSs after GKS, resemble the response of VSs following primary radiosurgery ${ }^{55}$. A transient increase in tumor volume occurred at a mean of 13.5 months (range 3-30 months), and should be distinguished from continuous growth, in order to prevent unnecessary interventions. The presence of central nonenhancement did not prove to be a prognostic factor indicating growth control, in contrary to what has been previously hypothesized ${ }^{29,56}$.

Table 5.6 Literature review of studies on planned subtotal resection and Gamma Knife surgery for large vestibular schwannomas.

$\begin{array}{lccccc}\text { Author } & \begin{array}{c}\text { Number } \\ \text { patients }\end{array} & \begin{array}{c}\text { Method of } \\ \text { measurement }\end{array} & \text { FU } & \text { Facial nerve } & \begin{array}{c}\text { Radiological } \\ \text { control rate }\end{array} \\ \text { Fuentes et al., 2008 } & 8 & \text { 2D } & 46 \text { (mean) } & 87.5 \% & 100 \% \\ \text { Iwai et al., 2003 } & 14 & \text { 2D } & \text { 32 (mean) } & 85.7 \% & 79 \% \\ \text { Park et al., 2006 } & 8 & \text { 2D } & 68.8 \text { (mean) } & \text { NR } & 100 \% \\ \text { Yang et al., 2008 } & 61 & \text { 2D } & 53.7 \text { (median) } & 95 \% & 93.5 \% \\ \text { Present series } & 50 & \text { Vol } & 33.8 \text { (median) } & 94 \% & 90 \%\end{array}$

$2 \mathrm{D}=$ two-dimensional VS measurements; Facial nerve=percentage of functional facial nerve preservation (House Brackmann Grade I and II); FU=Follow-up in months; NR=not reported; Vol=volume measurements. 


\section{Facial nerve function}

Although high rates of anatomical preservation rates have been published, functional outcomes in large series 1 year after complete resection of large VSs show HB Grades of I or II in $27-58 \%$ of the patients ${ }^{6-12}$ in whom the translabyrinthine ${ }^{7,9,10,12}$ and retrosigmoidal ${ }^{6-8,11}$ approaches were performed (Table 5.7). This is not surprising given that the facial nerve outcome in surgery is inversely related to VS size $e^{7,9,20,57}$. Furthermore, many patients in these series needed secondary facial nerve-related interventions. Mamikoglu et al. ${ }^{9}$ reported that $19 \%$ of surgically treated patients needed cranial nerve VII-cranial nerve XII anastomoses and that $30 \%$ needed gold weights to achieve adequate eye closure. Two studies reported on facial nerve outcome after subtotal resection of large VSs: HB Grade I or II was obtained in $82 \%$ and $88 \%$ of patients, respectively ${ }^{18,20}$. In large studies examining facial nerve function after primary GKS for small/medium VS, dysfunction occurs in $<1 \%$ of the cases $^{21,24-29}$; however, when performing radiosurgery for large VSs, facial nerve dysfunction is seen more often ${ }^{53}$. Results of the present study indicate that the preservation of facial nerve function is possible after subtotal microsurgery combined with GKS in large VSs. Good facial nerve function was retained in $94 \%$ of the patients and transient worsening of facial nerve function was observed after GKS in 2 of 50 patients. We showed that despite facial nerve monitoring and a "safe" subtotal resection, facial nerve neuropathies can occur, as also demonstrated in previous studies ${ }^{18,20}$. Intraoperative adverse events (such as excessive bleeding) or difficulties (adhesions) and cystic VS (impeding a decent dissection) can cause these "failures". However, the percentage of patients with HB Grade I or II facial nerve function in the present study exceeds the less favorable results published elsewhere, in which complete resection of large VS was performed. In three other studies reporting on facial nerve outcome after subtotal resection and postoperative GKS, rates comparable with those in the present study were reported, varying from 85.795\% ${ }^{30-32}$ (Table 5.6).

\section{Cochlear nerve function}

Patients with large VSs may present with serviceable hearing, because hearing function is not related to VS size ${ }^{58}$. Hearing preservation rates following microsurgery performed for large VSs in an attempt to maintain hearing are rather disappointing, varying from $0-29 \%$ (Table 5.7$)^{7,8,42-45}$. It is clear that hearing preservation, like facial nerve function, is strongly related to tumor size. Some authors state that in large VSs, hearing preservation is unlikely and should therefore not influence the choice of surgical approach ${ }^{10,12}$. In primary GKS for large VSs, the hearing preservation results vary from $38-94 \%^{50-53}$. However, patient numbers are limited in these studies. Although speculative due to a small cohort (2 patients), the results of the present study indicate that 
serviceable hearing preservation is possible after subtotal resection and GKS for large VSs. Hearing after subtotal resection and GKS in large VSs has been discussed only in the study of Yang et al. ${ }^{32}: 5$ of 10 patients with serviceable preoperative hearing maintained serviceable hearing postoperatively and 3 of these 5 patients maintained serviceable hearing after subsequent treatment with $\mathrm{GKS}^{32}$, but it was not specified how many of the 10 patients underwent hearing preserving surgery. Although no randomized controlled trial has been performed on the extent of VS resection and hearing preservation, the latter might be more likely when performing a subtotal resection rather than a complete resection, because traction and manipulation of the cochlear nerve is less likely to occur. In patients presenting with serviceable hearing in the context of a large VS, a retrosigmoidal resection should be performed along with intraoperative auditory brainstem response monitoring, in order to maintain a chance of hearing preservation.

\section{Treatment failure and complications}

Treatment failure occurred in 4 patients, and 3 of them were treated with a second GKS and 1 with a second microsurgical procedure followed by GKS for a larger residual VS. After a median follow-up of 14.5 months, the residual VSs remained stable or shrank. Previous studies have shown good response after second GKS for VS once the primary GKS has failed, with a very low risk of complications $^{59,60}$. One patient experienced an HB Grade III transient facial nerve paresis in the absence of lesion growth. One patient with a large progressive residual VS, was surgically treated and had a facial nerve paralysis postoperatively. Some authors find a higher risk of complications when dealing with additional surgery ${ }^{14,61}$. In our experience, second surgery was not necessarily related to a more difficult resection, as reconfirmed by others ${ }^{62}$. The number of complications during primary treatment was small (Table 5.5). One major complication, hemiparesis, occurred in 1 patient. The incidence of minor complications was lower as compared with their incidence in previous studies of complete resection in large VSs, especially regarding CSF leakages, wound infection and meningitis, ${ }^{6-8-12}$ (Table 5.7). A possible explanation for this is the decreased invasiveness and operating time of the resection. No studies have noted these complications following the subtotal resection of large VSs. No patient- or VS-related features could be associated with the occurrence of complications or the failure to control VS growth.

\section{Considerations and limitations}

Arguments against this combined technique might be that the patient is not completely cured in one operative session and that GKS might induce the malignant transformation of surrounding tissue. In our experience patients do not present major symptoms after GKS and patients can immediately resume 
their daily activities. The additional complications rate of GKS is low. The risk of malignant transformation has not been proven in a large study, but should lie between 1 in 1000 and 3 in 200.000 treated patients ${ }^{63,64}$. GKS is currently widely used, with excellent follow-up results, even in younger patients ${ }^{65}$.

This study also has some limitations. First, the follow-up time is relatively short. Given the median follow-up time (33.8 months) and the mean time of clinical failure (31.5 months), we acknowledge that a longer follow-up time is necessary to draw definite conclusions. The second limitation is inherent to a retrospective study with data collection from medical records, which carries the risk of selection bias in the incidence of morbidity.

Table 5.7 Results following complete microsurgical removal of large vestibular schwannomas larger than $3 \mathrm{~cm}$.

\begin{tabular}{|c|c|c|c|c|c|c|c|c|c|c|}
\hline Study & No. & Approach & $\begin{array}{l}\text { VS } \\
\text { Size } \\
(\mathrm{cm})\end{array}$ & $\begin{array}{c}\% \\
\text { cases } \\
\text { w/ CSF } \\
\text { leakage }\end{array}$ & $\begin{array}{c}\% \text { cases } \\
\text { w/ } \\
\text { meningitis }\end{array}$ & $\begin{array}{l}\% \text { cases } \\
\text { w/ wound } \\
\text { infection }\end{array}$ & $\begin{array}{l}\text { NVII }(\% \\
\text { cases) }\end{array}$ & $\begin{array}{c}\text { NVIII } \\
(\% \\
\text { cases) }\end{array}$ & $\begin{array}{l}\% \text { cases } \\
\text { of death }\end{array}$ & $\begin{array}{c}\% \text { cases } \\
\text { w/ } \\
\text { recurrence }\end{array}$ \\
\hline $\begin{array}{l}\text { Briggs et al. } \\
(1994)\end{array}$ & 167 & $\mathrm{TL}$ & $>4$ & 9.2 & 7.2 & NR & 42 & - & NR & NR \\
\hline $\begin{array}{l}\text { Jung et al. } \\
(2000)\end{array}$ & 30 & RS & $>4$ & 27 & 0 & NR & 42.1 & NR & 0 & NR \\
\hline $\begin{array}{l}\text { Lanman et al. } \\
\text { (1999) }\end{array}$ & 190 & $\mathrm{TL}$ & $>3$ & 14.2 & 3.7 & 1.1 & 52.6 & - & 0 & NR \\
\hline $\begin{array}{l}\text { Mamikoglu et al. } \\
\text { (2002) }\end{array}$ & 81 & $\mathrm{TL}$ & $>3 \& 4$ & 17 & 4 & NR & $27-58$ & - & 0 & $1 \%$ \\
\hline $\begin{array}{l}\text { Samii et al. } \\
(2009)\end{array}$ & 50 & RS & $>4$ & 6 & NR & NR & 44 & $11 \%$ & 0 & NR \\
\hline $\begin{array}{l}\text { Zhang et al. } \\
\text { (2005) }\end{array}$ & 105 & RS & $>4$ & 8.6 & 1.9 & 1 & 56.7 & NR & 1.9 & $3.5 \%$ \\
\hline $\begin{array}{l}\text { Wiet et al. } \\
(2001)\end{array}$ & 51 & RS\&TL & $>3.1$ & NR & NR & NR & 31 & $0(n=2)$ & NR & NR \\
\hline $\begin{array}{l}\text { Post et al. } \\
\text { (1995) }\end{array}$ & 4 & RS & $>3$ & NR & NR & NR & NR & 25 & NR & NR \\
\hline $\begin{array}{l}\text { Fischer et al. } \\
(1992)^{\star}\end{array}$ & 30 & RS & $>3$ & NR & NR & NR & NR & 20 & NR & NR \\
\hline $\begin{array}{l}\text { Samii et al. } \\
(2006)^{*}\end{array}$ & 92 & RS & $\mathrm{T} 4 \mathrm{a} / \mathrm{b}$ & NR & NR & NR & NR & $27-29$ & NR & $0.5 \%$ \\
\hline $\begin{array}{l}\text { Hecht et al. } \\
(1997)^{*}\end{array}$ & 2 & $\mathrm{RS} / \mathrm{MF}$ & $>3$ & NR & NR & NR & NR & 0 & NR & NR \\
\hline
\end{tabular}

MF-Middle fossa approach; NVII=preservation of good facial nerve function (HB Grade I or II) 1 year postoperatively; NVIII=preservation of serviceable hearing after last follow-up; RS: retrosigmoidal approach; T4a/b=Hannover classification for VS size: T4a=lesion compressing brain stem, T4b lesion deviating the $4^{\text {th }}$ ventricle; TL=translabyrinthine approach; No.: number. NR=not reported in study. * Data from large VS, extracted from studies evaluating outcome for various VS sizes. 


\section{Conclusions}

Given the good growth control and facial nerve function preservation, the possibility of preserving serviceable hearing and the low number of complications, a subtotal resection followed by GKS can be the treatment option of choice in large VS. Hearing preservation is possible, but the number

of patients with serviceable hearing in this study is too small to draw any conclusions. 


\section{References}

1. Stangerup SE, Caye-Thomasen $P$, Tos $M$, Thomsen J. The natural history of vestibular schwannoma. Otol Neurotol. 2006;27:547-52.

2. Shin YJ, Fraysse B, Cognard C, Gafsi I, Charlet JP, Berges C, Deguine O, Tremoulet M. Effectiveness of conservative management of acoustic neuromas. Am J Otol. 2000;21:857-62.

3. Kondziolka D, Lunsford LD, McLaughlin MR, Flickinger JC. Long-term outcomes after radiosurgery for acoustic neuromas. N Engl J Med. 1998;339:1426-33.

4. Flickinger JC, Kondziolka D, Niranjan A, Maitz A, Voynov G, Lunsford LD. Acoustic neuroma radiosurgery with marginal tumor doses of 12 to $13 \mathrm{~Gy}$. Int J Radiat Oncol Biol Phys. 2004;60:225-30.

5. Ito K, Shin M, Matsuzaki M, Sugasawa K, Sasaki T. Risk factors for neurological complications after acoustic neurinoma radiosurgery: refinement from further experiences. Int J Radiat Oncol Biol Phys. 2000;48:75-80.

6. Zhang X, Fei Z, Chen YJ, Fu LA, Zhang JN, Liu WP, He XS, Jiang XF. Facial nerve function after excision of large acoustic neuromas via the suboccipital retrosigmoid approach. J Clin Neurosci. 2005;12:405-8.

7. Wiet RJ, Mamikoglu B, Odom L, Hoistad DL. Long-term results of the first 500 cases of acoustic neuroma surgery. Otolaryngol Head Neck Surg. 2001;124: 645-51.

8. Samii M, Gerganov VM, Samii A. Functional outcome after complete surgical removal of giant vestibular schwannomas. J Neurosurg. 2010;112:860-7

9. Mamikoglu B, Wiet RJ, Esquivel CR. Translabyrinthine approach for the management of large and giant vestibular schwannomas. Otol Neurotol. 2002;23:224-7.

10. Lanman TH, Brackmann DE, Hitselberger WE, Subin B. Report of 190 consecutive cases of large acoustic tumors (vestibular schwannoma) removed via the translabyrinthine approach. J Neurosurg. 1999;90:617-23.

11. Jung S, Kang SS, Kim TS, Kim HJ, Jeong SK, Kim SC, Lee JK, Kim JH, Kim SH, Lee $\mathrm{JH}$. Current surgical results of retrosigmoid approach in extralarge vestibular schwannomas. Surg Neurol. 2000;53:370-7; discussion 7-8.

12. Briggs RJ, Luxford WM, Atkins JS, Jr., Hitselberger WE. Translabyrinthine removal of large acoustic neuromas. Neurosurgery. 1994;34:785-90; discussion 90-1.

13. Bloch DC, Oghalai JS, Jackler RK, Osofsky M, Pitts LH. The fate of the tumor remnant after less-than-complete acoustic neuroma resection. Otolaryngol Head Neck Surg. 2004;130:104-12.

14. El-Kashlan HK, Zeitoun H, Arts HA, Hoff JT, Telian SA. Recurrence of acoustic neuroma after incomplete resection. Am J Otol. 2000;21:389-92.

15. Godefroy WP, van der Mey AG, de Bruine FT, Hoekstra ER, Malessy MJ. Surgery for large vestibular schwannoma: residual tumor and outcome. Otol Neurotol. 2009;30:629-34.

16. Kameyama S, Tanaka R, Honda Y, Hasegawa A, Yamazaki H, Kawaguchi T. The long-term growth rate of residual acoustic neurinomas. Acta Neurochir (Wien). 1994;129:127-30.

17. Kameyama S, Tanaka R, Kawaguchi T, Honda $Y$, Yamazaki $H$, Hasegawa A. Long-term follow-up of the residual intracanalicular tumours after subtotal removal of acoustic neurinomas. Acta Neurochir (Wien). 1996;138:206-9.

18. Lownie SP, Drake CG. Radical intracapsular removal of acoustic neurinomas. Long-term follow-up review of 11 patients. J Neurosurg. 1991;74:422-5. 
19. Ohta S, Yokoyama T, Nishizawa S, Uemura K. Regrowth of the residual tumour after acoustic neurinoma surgery. Br J Neurosurg. 1998;12:419-22.

20. Park CK, Jung HW, Kim JE, Son YJ, Paek SH, Kim DG. Therapeutic strategy for large vestibular schwannomas. J Neurooncol. 2006;77:167-71.

21. Pollock BE, Lunsford LD, Flickinger JC, Clyde BL, Kondziolka D. Vestibular schwannoma management. Part I. Failed microsurgery and the role of delayed stereotactic radiosurgery. J Neurosurg. 1998;89:944-8.

22. Ramina R, Coelho Neto M, Bordignon KC, Mattei T, Clemente R, Pires Aguiar PH. Treatment of large and giant residual and recurrent vestibular schwannomas. Skull Base. 2007;17:109-17.

23. Sanna M, Falcioni M, Taibah A, De Donato G, Russo A, Piccirillo E. Treatment of residual vestibular schwannoma. Otol Neurotol. 2002;23:980-7.

24. Chung WY, Liu KD, Shiau CY, Wu HM, Wang LW, Guo WY, Ho DM, Pan DH. Gamma knife surgery for vestibular schwannoma: 10-year experience of 195 cases. J Neurosurg. 2005;102 Suppl:87-96.

25. Friedman WA, Bradshaw $P$, Myers A, Bova FJ. Linear accelerator radiosurgery for vestibular schwannomas. J Neurosurg. 2006;105:657-61.

26. Lunsford LD, Niranjan A, Flickinger JC, Maitz A, Kondziolka D. Radiosurgery of vestibular schwannomas: summary of experience in 829 cases. J Neurosurg. 2005;102 Suppl:195-9.

27. Pollock BE. Vestibular schwannoma management: an evidence-based comparison of stereotactic radiosurgery and microsurgical resection. Prog Neurol Surg. 2008;21:222-7.

28. Pollock BE, Link MJ. Vestibular schwannoma radiosurgery after previous surgical resection or stereotactic radiosurgery. Prog Neurol Surg. 2008;21:163-8.

29. Prasad D, Steiner M, Steiner L. Gamma surgery for vestibular schwannoma. J Neurosurg. 2000;92:745-59.

30. Fuentes S, Arkha Y, Pech-Gourg G, Grisoli F, Dufour H, Regis J. Management of large vestibular schwannomas by combined surgical resection and gamma knife radiosurgery. Prog Neurol Surg. 2008;21:79-82.

31. Iwai $\mathrm{Y}$, Yamanaka $\mathrm{K}$, Ishiguro $\mathrm{T}$. Surgery combined with radiosurgery of large acoustic neuromas. Surg Neurol. 2003;59:283-9; discussion 9-91.

32. Yang SY, Kim DG, Chung HT, Park SH, Paek SH, Jung HW. Evaluation of tumour response after gamma knife radiosurgery for residual vestibular schwannomas based on MRI morphological features. J Neurol Neurosurg Psychiatry. 2008; 79:431-6.

33. van de Langenberg R, de Bondt BJ, Nelemans PJ, Baumert BG, Stokroos RJ. Follow-up assessment of vestibular schwannomas: volume quantification versus two-dimensional measurements. Neuroradiology. 2009;51:517-24.

34. Koos WT, Day JD, Matula C, Levy DI. Neurotopographic considerations in the microsurgical treatment of small acoustic neurinomas. J Neurosurg. 1998;88: 506-12.

35. van de Langenberg R, Hanssens PE, Verheul JB, van Overbeeke JJ, Nelemans PJ, Dohmen AJ, de Bondt BJ, Stokroos RJ. Management of large vestibular schwannoma. Part II. Primary Gamma Knife surgery: radiological and clinical aspects. J Neurosurg. 2011;115:885-93.

36. Committee on Hearing and Equilibrium guidelines for the evaluation of hearing preservation in acoustic neuroma (vestibular schwannoma). American Academy of Otolaryngology-Head and Neck Surgery Foundation, INC. Otolaryngol Head Neck Surg. 1995;113:179-80. 
37. House JW, Brackmann DE. Facial nerve grading system. Otolaryngol Head Neck Surg. 1985;93:146-7.

38. Ferri GG, Modugno GC, Pirodda A, Fioravanti A, Calbucci F, Ceroni AR. Conservative management of vestibular schwannomas: an effective strategy. Laryngoscope. 2008;118:951-7.

39. Stangerup SE, Caye-Thomasen $\mathrm{P}$, Tos M, Thomsen J. Change in hearing during 'wait and scan' management of patients with vestibular schwannoma. The Journal of laryngology and otology. 2008;122:673-81.

40. Cross T, Sheard CE, Garrud P, Nikolopoulos TP, O'Donoghue GM. Impact of facial paralysis on patients with acoustic neuroma. Laryngoscope. 2000;110:1539-42.

41. Lee J, Fung K, Lownie SP, Parnes LS. Assessing impairment and disability of facial paralysis in patients with vestibular schwannoma. Archives of otolaryngology-head \& neck surgery. 2007;133:56-60.

42. Fischer G, Fischer C, Remond J. Hearing preservation in acoustic neurinoma surgery. J Neurosurg. 1992;76:910-7.

43. Hecht CS, Honrubia VF, Wiet RJ, Sims HS. Hearing preservation after acoustic neuroma resection with tumor size used as a clinical prognosticator. Laryngoscope. 1997; 107:1122-6.

44. Post KD, Eisenberg MB, Catalano PJ. Hearing preservation in vestibular schwannoma surgery: what factors influence outcome? J Neurosurg. 1995;83: 191-6.

45. Samii M, Gerganov V, Samii A. Improved preservation of hearing and facial nerve function in vestibular schwannoma surgery via the retrosigmoid approach in a series of 200 patients. J Neurosurg. 2006;105:527-35.

46. Grey PL, Moffat DA, Palmer CR, Hardy DG, Baguley DM. Factors which influence the facial nerve outcome in vestibular schwannoma surgery. Clin Otolaryngol Allied Sci. 1996;21:409-13.

47. Samii M, Matthies C. Management of 1000 vestibular schwannomas (acoustic neuromas): surgical management and results with an emphasis on complications and how to avoid them. Neurosurgery. 1997;40:11-21; discussion -3.

48. Cerullo L, Grutsch J, Osterdock R. Recurrence of vestibular (acoustic) schwannomas in surgical patients where preservation of facial and cochlear nerve is the priority. Br J Neurosurg. 1998;12:547-52.

49. Hasegawa T, Fujitani S, Katsumata S, Kida Y, Yoshimoto M, Koike J. Stereotactic radiosurgery for vestibular schwannomas: analysis of 317 patients followed more than 5 years. Neurosurgery. 2005;57:257-65; discussion -65.

50. Inoue HK. Low-dose radiosurgery for large vestibular schwannomas: long-term results of functional preservation. J Neurosurg. 2005;102 Suppl:111-3.

51. Lederman G, Lowry J, Wertheim S, Fine M, Lombardi E, Wronski M, Arbit E. Acoustic neuroma: potential benefits of fractionated stereotactic radiosurgery. Stereotact Funct Neurosurg. 1997;69(1-4 Pt 2):175-82.

52. Litvack ZN, Noren G, Chougule PB, Zheng Z. Preservation of functional hearing after gamma knife surgery for vestibular schwannoma. Neurosurg Focus. 2003; 14:e3.

53. Mandl ES, Meijer OW, Slotman BJ, Vandertop WP, Peerdeman SM. Stereotactic radiation therapy for large vestibular schwannomas. Radiother Oncol. 2010;95: 94-8.

54. Rowe JG, Radatz MW, Walton L, Hampshire A, Seaman S, Kemeny AA. Gamma knife stereotactic radiosurgery for unilateral acoustic neuromas. J Neurol Neurosurg Psychiatry. 2003;74:1536-42. 
55. Okunaga T, Matsuo T, Hayashi N, Hayashi Y, Shabani HK, Kaminogo M, Ochi M, Nagata I. Linear accelerator radiosurgery for vestibular schwannoma: measuring tumor volume changes on serial three-dimensional spoiled gradient-echo magnetic resonance images. J Neurosurg. 2005;103:53-8.

56. Linskey ME, Lunsford LD, Flickinger JC. Neuroimaging of acoustic nerve sheath tumors after stereotaxic radiosurgery. Ajnr. 1991;12:1165-75.

57. Lalwani AK, Butt FY, Jackler RK, Pitts LH, Yingling CD. Facial nerve outcome after acoustic neuroma surgery: a study from the era of cranial nerve monitoring. Otolaryngol Head Neck Surg. 1994;111:561-70.

58. van de Langenberg R, de Bondt BJ, Nelemans PJ, Dohmen AJ, Baumert BG, Stokroos RJ. Predictors of volumetric growth and auditory deterioration in vestibular schwannomas followed in a wait and scan policy. Otol Neurotol. 2011;32:338-44.

59. Yomo S, Arkha Y, Delsanti C, Roche PH, Thomassin JM, Regis J. Repeat gamma knife surgery for regrowth of vestibular schwannomas. Neurosurgery. 2009;64:4854; discussion -5 .

60. Liscak R, Vladyka V, Urgosik D, Simonova G, Vymazal J. Repeated treatment of vestibular schwannomas after gamma knife radiosurgery. Acta Neurochir (Wien). 2009;151:317-24; discussion 24.

61. Unger F, Walch C, Papaefthymiou G, Feichtinger K, Trummer M, Pendl G. Radiosurgery of residual and recurrent vestibular schwannomas. Acta Neurochir (Wien). 2002;144:671-6; discussion 6-7.

62. Pollock BE, Lunsford LD, Kondziolka D, Sekula R, Subach BR, Foote RL, Flickinger JC. Vestibular schwannoma management. Part II. Failed radiosurgery and the role of delayed microsurgery. J Neurosurg. 1998;89:949-55.

63. Ganz JC. Gamma knife radiosurgery and its possible relationship to malignancy: a review. J Neurosurg. 2002;97(5 Suppl):644-52.

64. Loeffler JS, Niemierko A, Chapman PH. Second tumors after radiosurgery: tip of the iceberg or a bump in the road? Neurosurgery. 2003;52:1436-40.

65. Lobato-Polo J, Kondziolka D, Zorro O, Kano H, Flickinger JC, Lunsford LD. Gamma knife radiosurgery in younger patients with vestibular schwannomas. Neurosurgery. 2009;65:294-300; discussion -1. 



\title{
MANAGEMENT OF LARGE VESTIBULAR SCHWANNOMA PART II. PRIMARY GAMMA KNIFE SURGERY: RADIOLOGICAL AND CLINICAL ASPECTS
}

\author{
Rick van de Langenberg \\ Patrick EJ Hanssens \\ Jeroen B Verheul \\ Jacobus J van Overbeeke \\ Patty J Nelemans \\ Amy JC Dohmen \\ Bert J de Bondt \\ Robert J Stokroos
}

Journal of Neurosurgery. 2011;115:885-893 


\section{Abstract}

\section{Object}

In large vestibular schwannomas (VSs), microsurgery is the main treatment option. A wait and scan policy or radiosurgery are generally not recommended given concerns of further lesion growth or increased mass effect due to transient swelling. Note, however, that some patients do not present with symptomatic mass effect or may still have serviceable hearing. Moreover, patients may be old, suffer from severe comorbidity, or refuse any surgery. In this study the authors report the results in patients with large VSs, primarily treated with Gamma Knife surgery (GKS), with special attention to volumetric growth, control rate and symptoms.

\section{Methods}

The authors retrospectively analyzed 33 consecutive patients who underwent GKS for large VSs, which were defined as $>6 \mathrm{~cm} 3$ and at least indenting the brainstem. Patients with neurofibromatosis Type 2 were excluded from analysis, as were patients who had undergone previous treatment. Volume measurements were performed on contrast enhanced T1-weighted MR images at the time of GKS and during follow-up. Medical charts were analyzed for clinical symptoms.

\section{Results}

Radiological growth control was achieved in $88 \%$ of cases, clinical control (that is, no need for further treatment) in $79 \%$ of the cases. The median follow-up was 30 months and the mean VS volume was $8.8 \mathrm{~cm}^{3}$ (range $6.1-17.7 \mathrm{~cm}^{3}$ ). No major complications occurred, although ventriculoperitoneal shunts were placed in 2 patients. The preservation of serviceable hearing and facial nerve and trigeminal nerve function was achieved in $58 \%, 91 \%$ and $86 \%$, respectively, with any facial and trigeminal neuropathy being transient. In $92 \%$ of the patients presenting with trigeminal hypesthesia before GKS, this resolved during follow-up. No patient- or VS-related feature was correlated with growth.

\section{Conclusion}

Primary GKS for large VSs leads to acceptable growth and clinical control rates, with the chance of hearing preservation. Although a higher incidence of clinical control failure and postradiosurgical morbidity is noted, as compared with that for smaller VS, primary radiosurgery is suitable for a selected group of patients. Absence of symptomatology due to mass effect on the brain stem or cerebellum is essential, as are close clinical and radiological follow-ups, because there is little reserve for growth or swelling. 


\section{Introduction}

Currently, radiosurgery is regarded as an optimal treatment regimen for small and medium-sized vestibular schwannomas (VSs). Excellent long-term growth control combined with the preservation of function of trigeminal and facial nerve function and hearing has been reported ${ }^{1-9}$. A treatment dilemma arises, however, when dealing with large VSs. A wait and scan policy is generally not recommended given the possibility of life-threatening complications due to further VS progression. Moreover, tumor volume can increase as a result of radiosurgery-induced acute transient VS swelling. Therefore, radiosurgery is often avoided in these cases. Surgery is usually advised as treatment option in large VS. When performing complete microsurgical removal in these cases, a considerable risk of a suboptimal facial nerve outcome exists and complications are not uncommon ${ }^{10-19}$.

But some patients may present without disabling symptoms. Despite the fact that a large VS is present in the cerebellopontine angle, for example, a patient may not show symptomatology necessitating surgical intervention. In addition, they may present with serviceable hearing. In large VSs, hearing preservation results seem more favorable after radiosurgery ${ }^{20-22}$ than after microsurgery ${ }^{15,16,23-26}$. Some patients may be old or may present with significant comorbidity, making them less than ideal candidates for VS surgery. Finally, some patients may refuse to undergo surgery. Therefore, some patients presenting with large VSs without symptomatic mass effect are candidates for primary radiosurgery. Few studies, all with a relatively small number of patients, report on outcome after GKS in large VSs, ${ }^{2,720-22,27-29}$, and all but two $^{2,29}$ utilized $2 \mathrm{D}$ measurements, which are less reliable compared to volume measurements ${ }^{30}$.

We performed the present study to evaluate GKS in a selected group of patients harboring large VSs and to focus on the volumetric growth, control rate and clinical symptoms after radiosurgery.

\section{Methods}

\section{Patients population}

All patients receiving primary GKS for a large VS larger than $6 \mathrm{~cm}^{3}$ between January 1, 2002 January 1, 2009, were retrospectively analyzed. All VSs at least indented the brain stem, according to the Koos classification (Figure 6.1A and $6.1 \mathrm{C})^{31}$. 


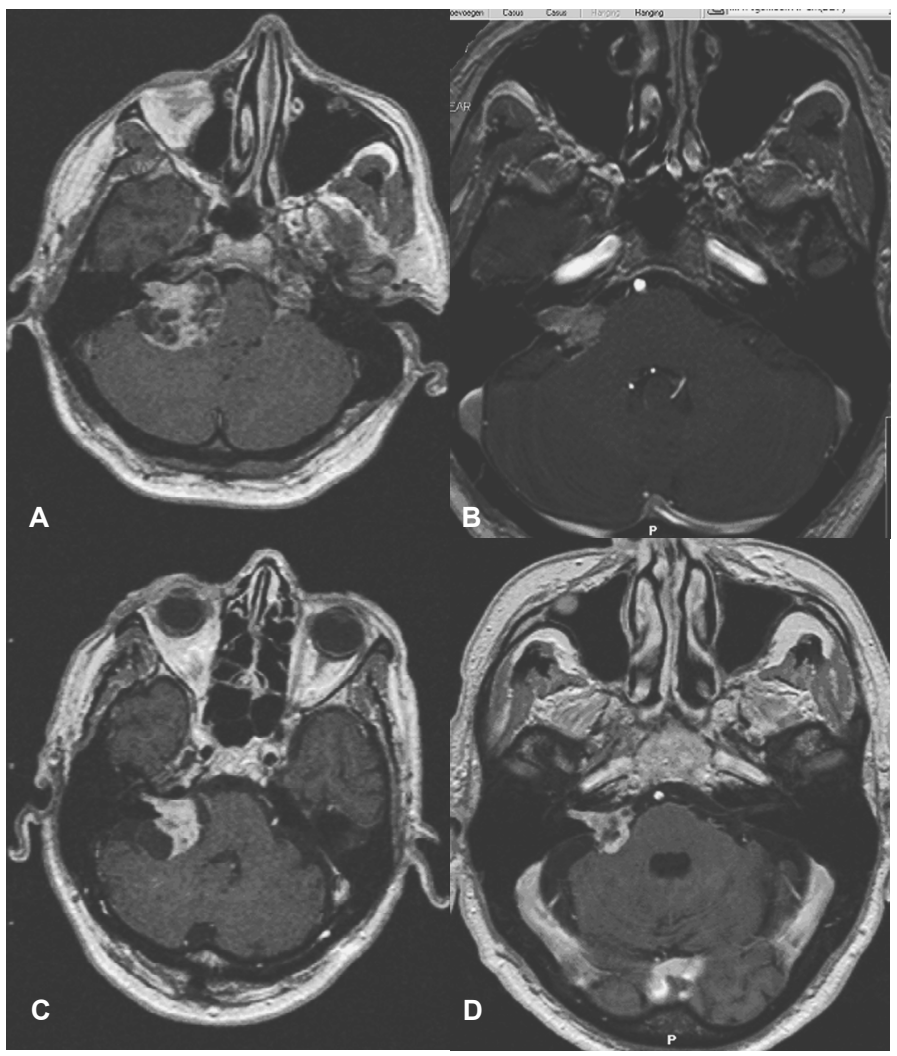

Figure 6.1 Axial contrast enhanced T1-weighted MR images (A and B) obtained in a 47-year-old patient with a large $\left(17.7 \mathrm{~cm}^{3}\right)$ vestibular schwannoma (VS), Koos Grade IV. The patient reported complete unilateral hearing loss and hypesthesia of the maxillary branch of the trigeminal nerve (A). The patients specifically chose treatment with primary Gamma Knife surgery (GKS). Twenty-four months after GKS the tumor volume was reduced to $4.6 \mathrm{~cm}^{3}(\mathbf{B})$. Sensibility in the maxillary branch recovered completely and the patient is functioning well.

Axial contrast enhanced T1-weighted MR images ( $\mathbf{C}$ and $\mathbf{D}$ ) obtained in a 83-year-old patient, with a large cystic VS (12.9cm3, Koos Grade IV, [C]). Surgical intervention was not recommended because of severe comorbidity. Therefore, primary GKS was initiated. Preoperative symptoms consisted of unilateral deafness and hypesthesia of the maxillary branch of the trigeminal nerve. After a follow-up of 24 months a significant volume reduction occurred (last volume $2.5 \mathrm{~cm} 3$, [D]). The patient is functioning well, and sensibility in the trigeminal nerve fully recovered.

Patients who had undergone microsurgery (16 patients) were excluded from our analysis, leaving 41 patients who underwent primary GKS at the GammaKnife Center, Tilburg. Five patients with neurofibromatosis Type 2 were also excluded and 3 patients were lost to follow-up, leaving a total of 33 patients for study inclusion. The diagnosis of VS was made on a clinical and radiological basis. Patients with Koos Grade III VSs were treated after lesion 
growth was seen after 1 year follow-up, whereas intervention without follow-up was planned in patients with Koos Grade IV patients. Only patients with VS $<4 \mathrm{~cm}$ maximum extracanalicular dimension were eligible for primary GKS, because some degree of symptomatic mass effect is usually present in larger VS. Patients were informed about the diminished reserve to observe growth or transient swelling after radiosurgery, possibly resulting in a higher chance of treatment failure. A subtotal resection followed by GKS for residual VS was also discussed as a possibility. If patients presented with symptomatic mass effect (cerebellar signs such as dysmetria and truncal ataxia or long tract signs such as hemiplegia), they were not considered as candidates for primary GKS; if symptomatic mass effect was present, a subtotal microsurgical removal followed by postoperative GKS was the therapy of choice. These results are published separately ${ }^{32}$.

\section{Surgical technique}

Stereotactic radiosurgery was performed using a Leksell Gamma Knife 4C (Elekta $\mathrm{AB}$ ). Treatment planning was completed with Leksell GammaPlan software (version 8.3.1). A dose of 12.5 to $13.0 \mathrm{~Gy}$ (mean $12.6 \mathrm{~Gy}$ ) was prescribed to the isodose, covering $90 \%$ of the tumor volume. The maximum dose varied from 18.1 to $25.5 \mathrm{~Gy}$ (mean $20.79 \mathrm{~Gy}$ ). The tumor margin dose varied from 10.3 to $13 \mathrm{~Gy}$ (mean $11.6 \mathrm{~Gy}$ ). The number of isocenters per patient varied from 3 to 23 (mean 9 isocenters). All patients received 10 milligram dexamethasone intravenously prior to GKS; the dose was tapered off according to a 12-day schedule. All patients could leave the hospital the same day of surgery and resume their daily activities. Follow-up was planned biannually for the first 2 years and every year thereafter. A clinical chart review was performed to obtain information at presentation and after GKS.

Cranial neuropathy was defined as transient or permanent nerve dysfunction. Audiometric assessments were recorded according to the American Academy of Otolaryngology- Head and Neck Surgery guidelines and consisted of a mean pure tone average $(0.5,1,2$ and $4 \mathrm{kHz})$ and speech discrimination score (\%). Patients were assigned to hearing classes $A, B, C$ or $D$, where $A$ and $B$ were considered as serviceable hearing and $C$ and $D$ were not ${ }^{33}$. Facial nerve function was scored according to the House Brackmann scale $(H B)^{34}$. HB scores included in this study were noted at the first visit to the outpatient department at presentation and 1 year after GKS. Good postoperative facial nerve function was defined as $\mathrm{HB}$ I or II.

One reader experienced in VS imaging performed all radiological evaluations on axial Gd-enhanced ( $0.1 \mathrm{mg} / \mathrm{kg}$ body weight) T1-weighted MR images. The slice thickness was usually 1 or 2 millimeter, there was no slice gap. Volume measurements were performed with Leksell GammaPlan 8.3.1. Vestibular schwannoma characteristics were noted as follows: central nonenhancement 
was defined as a hypointense area in the center of a VS on contrast enhanced T1-weighted MR imaging. Cystic VSs were defined as VSs with enhanced cysts making up at least one-third of the nonenhancement of the tumor volume on contrast enhanced T1-weighted and enhancing on T2-weighted images.

Clinical VS control was defined as no need for further treatment (either GKS or microsurgery) following primary GKS. Radiological control was defined as VS regression or absence of significant growth beyond measurement error, which previously has been defined as a difference of $5.7 \%$ between subsequent scans for large $\mathrm{VS}^{30}$.

\section{Statistical analysis}

Absolute changes in volume were calculated by subtracting the VS volume at the time of GKS from its volume at the last measurement (volume at $t_{x}$-volume at $t_{0}$ ). Relative changes in volume were calculated as the absolute change divided by the VS volume before GKS $\left(t_{0}\right)$ : (volume at $t_{x}$-volume at $t_{0}$ ) / volume at $t_{0}$. This relative change was multiplied by 100 to achieve a percentage change in volume. The cumulative probability of clinical and radiological control was calculated using Kaplan-Meier analyses. Differences in mean values were tested for significance using the independent $\mathrm{t}$-test. Fisher exact and $\chi^{2}$ test were used for categorical data. A Cox proportional hazards model was used to analyze differences in clinical and radiological control between the patient group receiving a subtotal resection followed by $\mathrm{GKS}^{32}$ and the group undergoing primary GKS. For all tests, P-values $<0.05$ indicated statistical significance. All analyses were performed using SPSS 15.0 statistical software (SPSS, Chicago, II, USA).

\section{Results}

\section{Patient and intervention characteristics}

There were 15 males and 18 females, with a mean age of 54.8 years (range 30-83 years). All patients reported hearing loss on presentation, whereas 12 $(36 \%)$ of 33 patients presented with serviceable hearing (Class A and B). Twenty-three $(70 \%)$ patients presented with co-existing tinnitus, $17(51 \%)$ with vertigo and $12(36 \%)$ showed trigeminal hypesthesia. One patient presented with a long history of HB Grade II facial paresis. One patient presented primarily with diplopia, because of an abducens nerve paresis due to VS compression. None of the patients presented with symptomatic mass effect. All presented with a large, unilateral VS, with a mean volume of $8.8 \mathrm{~cm}^{3}$ (range $6.1-17.7 \mathrm{~cm}^{3}$ ). Before GKS, 1 patient underwent ventriculoperitoneal drainage, and one patient received lumboperitoneal drainage, because of symptomatic 
secondary hydrocephalus. Primary GKS was performed in 12 patients because of serviceable hearing, in 17 patients because of patient preference and in 4 patients because of significant comorbidity. Further clinical and radiological VS features seen at presentation are listed in Table 6.1.

Table 6.1 Clinical and radiological vestibular schwannoma characteristics at presentation.

\begin{tabular}{|c|c|}
\hline Characteristic & No. \\
\hline Men / Women & $15 / 18$ \\
\hline Mean age in yrs (range) & $54.8(30-83)$ \\
\hline Median follow-up in mos. (range) & $30(12-72)$ \\
\hline \multicolumn{2}{|l|}{ Symptoms (\%) } \\
\hline Tinnitus & $23(70)$ \\
\hline Vertigo & $17(51)$ \\
\hline Trigeminal hypesthesia & $12(35)$ \\
\hline Facial weakness (HBII) & $1(3)$ \\
\hline Ataxia & $0(0)$ \\
\hline Symptomatic hydrocephalus necessitating drainage & $2(6)$ \\
\hline Ventriculoperitoneal drainage & $1(3)$ \\
\hline Lumbar drainage & $1(3)$ \\
\hline Papilledema & $0(0)$ \\
\hline \multicolumn{2}{|l|}{ Hearing class } \\
\hline A & $3(9)$ \\
\hline B & $9(27)$ \\
\hline C & $10(30)$ \\
\hline $\mathrm{D}$ & $11(34)$ \\
\hline \multicolumn{2}{|l|}{ VS Characteristics } \\
\hline \multicolumn{2}{|l|}{ VS Side } \\
\hline Left & $16(48)$ \\
\hline Right & $17(52)$ \\
\hline Cystic VS & $4(12)$ \\
\hline Volume $(\mathrm{cm} 3)$ & $8.8(6.1-17.7)$ \\
\hline Diameter $(\mathrm{cm})$ & $3.0(2.3-4.0)$ \\
\hline \multicolumn{2}{|l|}{ Extracanalicular dimension in $\mathrm{cm}$} \\
\hline $2-3$ & 15 \\
\hline $3-4$ & 18 \\
\hline$>4$ & 0 \\
\hline \multicolumn{2}{|l|}{ Koos grade } \\
\hline I-II & 0 \\
\hline III & 10 \\
\hline IV & 23 \\
\hline \multicolumn{2}{|l|}{ Hannover classification } \\
\hline $\mathrm{T} 1-3 \mathrm{~b}$ & 0 \\
\hline $4 a$ & 10 \\
\hline $4 b$ & 23 \\
\hline
\end{tabular}

Hearing class according to the American Academy of Otolaryngology - Head and Neck. Surgery guidelines, in which hearing class $A$ and $B$ are considered as serviceable and $C$ and $D$ not. $H B=$ House Brackmann classification for facial nerve function. Koos gradation: Grade I: intracanalicular VS, Grade II: extending into the cerebellopontine angle, Grade III: compressing the brain stem, Grade IV: deviating the $4^{\text {th }}$ ventricle. Hannover classification: Class T1, intracanalicular VS; Class T2, intra- and extracanalicular tumor; Class T3a, filling the cerebellopontine cistern; Class T3b, VS reaching the brainstem; Class T4a, VS compressing the brainstem; and Class T4b, VS severely dislocating the brainstem and compressing the $4^{\text {th }}$ ventricle. VS: vestibular schwannoma; Mos.: months. HB: House Brackmann score for grading facial nerve function. 


\section{Clinical and radiological control}

Significant VS growth was seen in 4 of 33 patients, resulting in a radiological control rate of $88 \%$ of patients. Clinical VS control was achieved in $26(79 \%)$ of 33 cases (Table 6.2). Median follow-up was 30.0 months (range 12-72 months). The cumulative probability of clinical and radiological control is shown in the Kaplan-Meier analyses (Figures 6.2 and 6.3). No deaths occurred during the follow-up. Clinical treatment failure occurred after a mean of 35 months (range 12-52 months). No patient- or VS-related characteristic (age, sex, location, baseline VS size, central nonenhancement, presence of cystic components) predicted clinical treatment failure, radiological treatment failure or complications ( $P=$ not significant). Two of the 7 clinical failures were treated with a second GKS, and in 5 patients subtotal surgical resection followed by second GKS was performed (Table 6.3). No complications occurred after second GKS. In 1 patient who received a retrosigmoidal subtotal resection, a facial paralysis occurred despite facial nerve monitoring and microsurgical precision. A sural nerve anastomosis was performed, finally leading to an HB Grade IV paresis. The other 4 patients underwent a translabyrinthine subtotal resection and subsequent GKS without complications. After a median period of 12 months after second intervention, no VS growth was seen.

Table 6.2 Control characteristics.

\section{Control rate}

Radiological control rate

Clinical control rate

Second GKRS

MS followed by GKRS
No. (\%)

$29(88 \%)$

$26(79 \%)$

$2(6 \%)$

$5(15 \%)$

GKS=Gamma Knife surgery. MS=microsurgery.

Table 6.3 Characteristics of patients undergoing second intervention after primary Gamma Knife surgery for a large vestibular schwannoma.

\begin{tabular}{|c|c|c|c|c|c|c|}
\hline $\begin{array}{l}\text { Case } \\
\text { no. }\end{array}$ & $\begin{array}{l}\text { Size before } \\
\text { GKS }(\mathrm{cm} 3)\end{array}$ & $\begin{array}{l}\text { Size before } 2^{\text {nd }} \\
\text { intervention } \\
(\mathrm{cm} 3)\end{array}$ & $\begin{array}{l}\text { Time between GKS } \\
\& 2^{\text {nd }} \text { intervention } \\
\text { (mos) }\end{array}$ & $\begin{array}{l}\text { Growth } \\
\text { pattern }\end{array}$ & $\begin{array}{c}\text { New symptoms } \\
\text { during FU }\end{array}$ & Intervention \\
\hline 1 & 6.8 & 9.5 & 22 & $1^{*}$ & None & MS/GKS \\
\hline 2 & 6.9 & 16.4 & 36 & 1 & NV & MS/GKS \\
\hline 3 & 7.2 & 7.2 & 12 & 2 & $\begin{array}{c}\text { NII, papilledema, } \\
\text { ataxia }\end{array}$ & MS/GKS \\
\hline 4 & 9 & 7 & 42 & 3 & NV & MS/GKS \\
\hline 5 & 6 & 8.3 & 30 & 4 & None & GKS \\
\hline 6 & 9.8 & 10.5 & 50 & 4 & None & MS/GKS \\
\hline 7 & 13.8 & 13 & 52 & 3 & None & GKS ** \\
\hline
\end{tabular}

Growth pattern: 1: continuous growth, 2: stable VS, 3: shrinkage, followed by regrowth not beyond baseline volume, 4 shrinkage, followed by regrowth beyond baseline volume. FU=Follow-up. Intervention: MS=Microsurgery; GKS=Gamma Knife surgery; Mos=months; NII=cranial nerve II; $\mathrm{NV}=$ cranial nerve $\mathrm{V}$. * This could have been transient swelling ** Patient refusing microsurgery. 


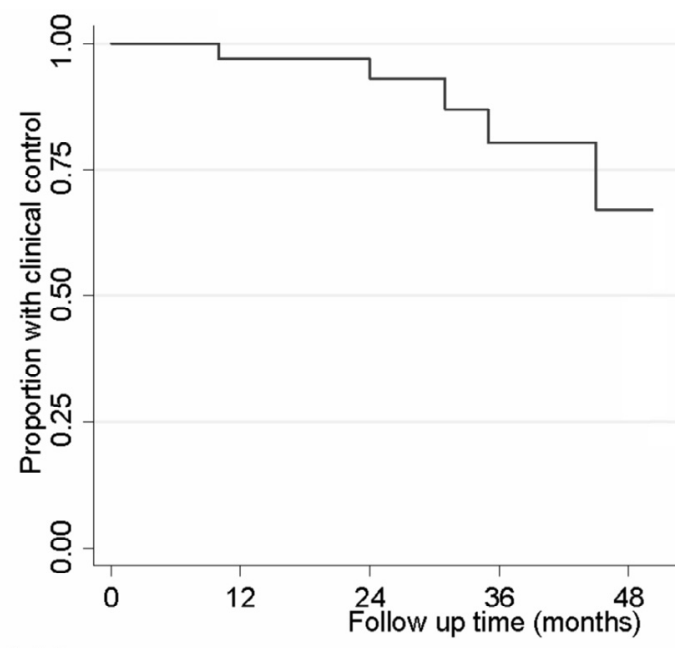

Number at risk

$\begin{array}{lllll}33 & 31 & 25 & 10 & 4\end{array}$

Figure 6.2 Kaplan-Meier analysis of clinical control during follow-up after primary Gamma Knife surgery in large vestibular schwannomas.

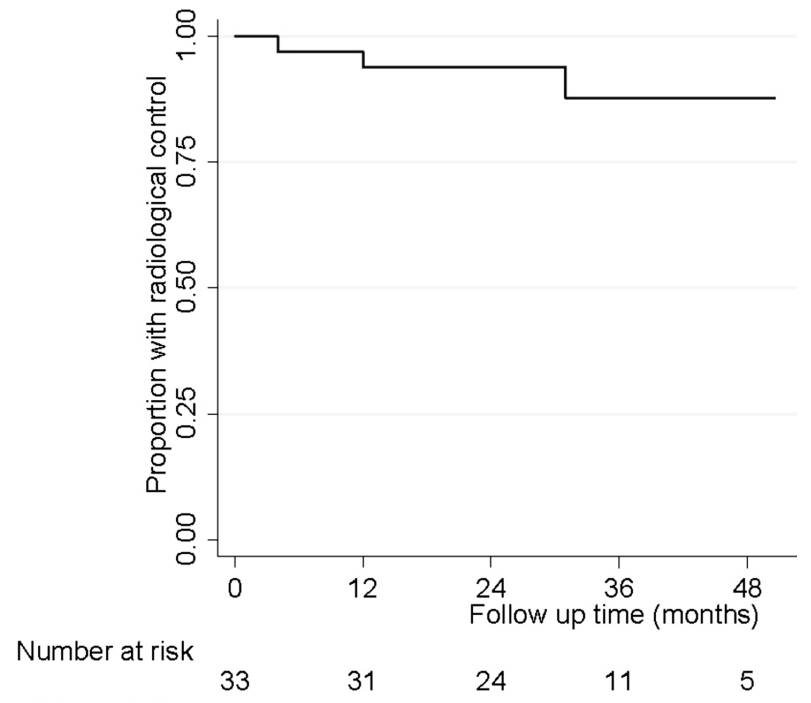

Figure 6.3 Kaplan-Meier analysis of radiological growth control during follow-up after primary Gamma Knife surgery in large vestibular schwannomas. 


\section{Cranial nerve preservation}

One year post-GKS, good facial nerve function (HB Grades I and II) was preserved in $100 \%$ of the patients (Table 6.4). In the 12 patients presenting with serviceable hearing, 7 (58\%) retained their functional hearing during a median follow-up of 37.2 months (Table 6.4). Of the 12 patients experiencing trigeminal hypesthesia at diagnosis, $11(92 \%)$ resolved of their condition during the follow-up. The abducens paresis in 1 patient resolved after GKS and the VS shrank.

Table 6.4 Distribution of facial nerve and cochlear function in 33 patients undergoing primary Gamma Knife radiosurgery because of a large vestibular schwannoma.

\begin{tabular}{lcc} 
House Brackmann & Pre GKS visit & One year after GKS \\
Grade & No $(\%)$ & No $(\%)$ \\
\hline I & $32(97)$ & $32(97)$ \\
II & $1(3)$ & $1(3)$ \\
III & $0(0)$ & $0(0)$ \\
IV & $0(0)$ & $0(0)$ \\
V & $0(0)$ & $0(0)$ \\
VI & $0(0)$ & $0(0)$ \\
Hearing class & $3(9)$ & $2(6)$ \\
A & $9(27)$ & $5(15)$ \\
B & $10(30)$ & $9(27)$ \\
C & $11(34)$ & $17(52)$ \\
D & Last FU after GKS
\end{tabular}

Facial nerve function is graded according to the House-Brackmann facial nerve grading system and cochlear function according to American Academy of Otolaryngology - Head and Neck Surgery guidelines, in which hearing class $A$ and $B$ are considered as serviceable and $C$ and $D$ not. $\mathrm{FU}=$ follow-up, $\mathrm{GKS}=$ Gamma Knife surgery.

\section{Patient complications}

There were no major complications after GKS, although minor complications did occur (Table 6.5): 2 patients experienced transient HB Grade II facial paresis. Three patients experienced transient facial hypesthesia and 1 of them patient experienced progressive VS growth for which surgical intervention was necessary. Two patients needed ventriculoperitoneal drainage because of symptomatic hydrocephalus, 6 and 12 months after GKS, respectively, even in the absence of significant lesion growth. Although there was no significant VS growth, papilledema and progressive ataxic gait developed in 1 patient and microsurgical subtotal removal followed by GKS was finally performed. Additional steroid treatment was needed after GKS in $6(18 \%)$ of 33 patients with new or worsened neurological symptoms. 
Table 6.5 Complications after Gamma Knife surgery.

$\begin{array}{lc}\text { Complication } & \text { No. / Total (\%) } \\ \text { Minor } & \\ \text { Transient facial paresis } & 3 / 33(9) \\ \text { Transient facial hypesthesia } & 3 / 21(14) \\ \text { Symptomatic hydrocephalus } & 2 / 31(6) \\ \text { VP drain } & 2 / 31(6) \\ \text { Long tract symptoms (ataxia) } & 1(3) \\ \text { Transient dysfunction cranial nerve IX, X } & 0(0) \\ \text { Major } & \\ \text { Hemiparesis } & 0(0) \\ \text { Death } & 0(0)\end{array}$

$\mathrm{FU}=$ follow-up, $\mathrm{VP}=$ ventriculoperitoneal drainage.

\section{Postradiosurgical growth patterns}

After GKS, 3 volume-changing patterns were identified: 1) shrinkage (22 [67\%] of 33 lesions), which was subdivided into direct shrinkage (18 [55\%] of 33 lesions; Figure 6.1.B and 6.1.D) and transient swelling followed by shrinkage (4 [12\%] of 33 lesions); 2 ) stability (7 [ 21\%] of 33 lesions), which was subdivided into stable VS (5 [15\%] of 33 lesions) and shrinkage followed by regrowth not above the baseline volume (2 [6\%] of 33 lesions); and 3 ) growth (4 [12\%] of 33 lesions), which was further subdivided into continuous growth (2 [6\%] of 33 lesions) and shrinkage followed by regrowth above the baseline volume (2 [6\%] of 33 lesions). Transient swelling occurred after a mean of 8 months after GKS (range 6-12 months). Regrowth following shrinkage was observed after a mean of 37 months (range 24-48 months). Central nonenhancement was seen in $30(91 \%)$ of 33 VSs after GKS. Five $(83 \%)$ of 6 patients with growth (continuous or after shrinkage), showed central nonenhancement, as compared with 25 (93\%) of 27 without growth, which is not statistically significant $(P=0.46)$. Four patients showed cystic components at the start of GKS. During follow-up, the lesions shrank in 2 patients and remained stable in 2.

\section{Discussion}

In large VSs, intervention is often necessary because life-threatening symptomatology can occur as a result of further tumor growth. Radiosurgery is generally not performed given the risk of acute transient swelling, which can lead to additional symptomatic brainstem compression. Microsurgery is generally considered the treatment of choice. However, when performing complete microsurgical removal of large VSs, complications occur frequently, a suboptimal facial nerve outcome is the rule more than the exception ${ }^{10-17}$ and 
the preservation of serviceable hearing (if present) is not likely to occur ${ }^{15,16,23-26}$. In anticipation of these suboptimal facial nerve outcomes, some authors have opted to perform a subtotal resection to reduce the risk of facial nerve dysfunction, followed by GKS for remnant tumor to reduce the chance of lesion regrowth ${ }^{32,35}$. We reported on subtotal resection followed by GKS for large symptomatic VSs, with a $92 \%$ clinical control rate, a $94 \%$ rate of good facial nerve function preservation during follow-up and a minimal risk of complications $^{32}$. Nonetheless, risk of death in VS surgery, which has been reduced the past decades in response to superior microsurgical skills, is around $1 \%$, even at the most experienced centers ${ }^{18,19}$.

Some patients may present with a large VS on MR imaging but without disabling symptoms and mass effect. In addition, serviceable hearing may be present. Other patients may be physically unfit to undergo VS microsurgery as a result of severe comorbidity. Large studies have shown excellent results for growth control in small/medium VSs after primary GKS with minimal morbidity $^{1-9}$. This fact supported our rationale for the treatment featured in the present study. In a selected group of patients presenting with large VSs without symptoms of mass effect, microsurgical resection can be avoided and primary GKS can control lesion growth and better preserve cranial nerve function.

\section{Growth control}

A limited number of studies on radiosurgery as the primary treatment for large VSs have been conducted ${ }^{2,7,20-22,27-29}$ (Table 6.6). Growth control rates vary from $57 \%$ to $100 \%$. Measurements were performed with less reliable 2D measurements in nearly all of these studies ${ }^{7,20-22,27,28}$ and only 2 studies used volume measurements ${ }^{2,29}$. In addition, patients with previous VS-related treatments were included in all the studies, as were follow-ups with CT scanning ${ }^{2,20,28}$ and patients with neurofibromatosis Type $2^{20,28}$. Therefore, a direct comparison of these studies is difficult. These confounders are not part of the current study. Radiological control was achieved in $88 \%$ of the patients and clinical control was achieved in $79 \%$. The lower clinical control rate was due to several reasons, illustrating the diminished reserve to observe growth or transient swelling of large VSs after GKS. First, one patient underwent surgery in the absence of lesion growth because of severe symptomatology. Second, 2 patients had interventions because of tumor regrowth, not above starting volume, without symptomatology. Note that in the absence of life-threatening symptomatology (Table 6.3), 4 patients underwent interventions based on radiological growth, not above the starting volume per se. A more reserved attitude toward intervention in case of asymptomatic growth - by performing MR imaging and clinical follow-up more frequently and by taking into account the possibility of transient swelling - might on the one hand have provided a better clinical control rate of up to $91 \%$, exceeding the radiological growth control rate. 


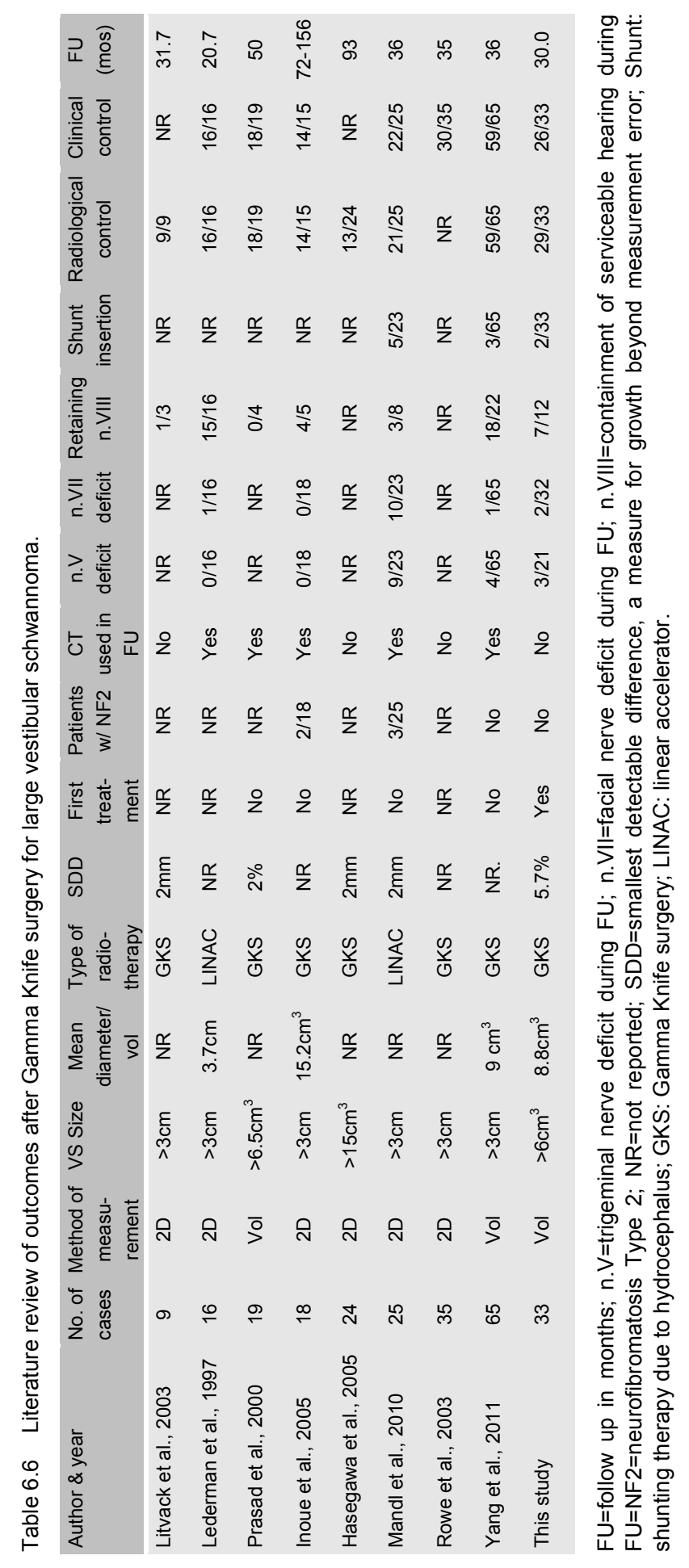


On the other hand, this could carry a higher risk of life-threatening symptoms. Our results are comparable with those in other studies reporting radiological growth control in large VSs. Radiological control was seen in $87 \%$ of the published cases $(150 / 173)^{2,7,20-22,28,29}$. There was no difference between clinical and radiological control, as seen in the present series. Two studies reported on worse growth control in the largest subset of large VS, which was not the case in our series ${ }^{7,29}$. No patient- or VS-related factors predicted radiological or clinical failure.

Three main radiological growth patterns were identified: 1) shrinkage $(67 \%)$, subdivided into direct shrinkage $(55 \%)$ and transient swelling followed by shrinkage (12\%); 2 ) stability (12\%), subdivided into continuous stable VS (15\%) and shrinkage followed by regrowth not above baseline volume (6\%); and 3 ) growth $(12 \%)$, which was subdivided in continuous growth $(6 \%)$ and shrinkage followed by regrowth above baseline $(6 \%)$. Transient swelling occurred at a mean of 8 months (range 6-12 months) and, as mentioned before, should not be confused with continuous growth to prevent unnecessary interventions. Regrowth after shrinkage was observed after a mean of 37 months (range 2448 months). Central nonenhancement, a reaction due to GKS and previously defined as good prognostic factor regarding growth control ${ }^{2,36}$, did not, in fact, prove to be a prognostic factor indicating growth control. Cystic VS have been regarded as difficult to treat with GKS because of the increased risk of rapid volume increase ${ }^{37-39}$, which was not seen in the present study. The volumetrical response of large VSs after GKS resembles the response of small/medium VSs in primary radiosurgery ${ }^{40}$. No patient- or VS-related correlated with treatment failure.

\section{Cranial nerve morbidity and complications}

During follow-up, serviceable hearing and facial nerve and trigeminal nerve function were preserved in $58 \%, 91 \%$ and $86 \%$, respectively (Tables $6.4 \& 6.5$ ). All new onset facial and trigeminal nerve neuropathies were transient. Ninetytwo percent of the patients complaining of facial hypesthesia pre-GKS recovered during follow-up. Because of symptomatic secondary hydrocephalus, 2 patients needed ventriculoperitoneal drainage after GKS, despite the absence of significant tumor growth. Rates of facial and trigeminal nerve neuropathies, as well as the occurrence of hydrocephalus for which drainage was necessary, are higher compared to rates after GKS in smallmedium VS, where these are described in $<1 \%$ of the cases ${ }^{1-9}$. Risk factors for trigeminal and facial neuropathies include radiation doses and VS volume ${ }^{41-43}$. As shown in Table 6.6, few studies have reported on cranial nerve preservation after GKS in large VSs, making comparisons difficult. Clearly, facial nerve outcome is superior, compared with the results following complete microsurgical removal of large VSs in which good function (HB Grade I or II) is 
reported in $28-58 \%$ of the cases ${ }^{10-17}$. Facial nerve outcome is comparable with that in studies featuring subtotal resection followed by $\mathrm{GKS}^{32,35,44-46}$.

Only 6 studies have documented serviceable hearing preservation after primary GKS in large VS. Forty-one $(71 \%)$ of the 58 patients in these studies retained serviceable hearing after follow-up ${ }^{2,20-22,28,29}$. These results are in line with those obtained in the present study and are superior to the published range of hearing preservation rates following complete microsurgical removal of large VSs $(0 \%-29 \%)^{15,16,23-26}$. Hearing loss after GKS is caused by the toxic effects of GKS on the cochlea ${ }^{47,48}$, which is almost unavoidable when planning for GKS. If possible, the treatment plan is modified to minimize the dosage to the cochlea. No large studies have documented hearing preservation after subtotal resection. No patient or VS related factors predicted complications.

\section{Study considerations}

Primary GKS for large VSs has shown reasonable growth control and complication rates in the present series of selected patients. Arguments against this treatment strategy are that radiosurgery causes an increased risk of complications as a result of swelling and that microsurgery following GKS would be more complicated than primary microsurgery. Indeed, an increased postradiosurgical morbidity rate was seen after GKS, when compared with GKS in smaller sized VS ${ }^{1-9}$. When choosing GKS for large VS, extensive patient instructions are obligatory before radiosurgery, as are close clinical and radiological follow-ups at least every 6 months in the first 2 years after the procedure. Previous authors found no clear relationship between prior radiosurgery and a subsequent difficulty in resecting a $V S^{49,50}$, which has also been our experience. A subtotal resection is advised in the event that one needs intervention after primary $\mathrm{GKS}^{50,51}$. No procedural complications occurred in 4 of 5 patients who underwent microsurgery in this series. In 1 patient a facial nerve paralysis occurred for which a sural nerve anastomosis was performed. In case of treatment failure, a second GKS treatment can be performed effectively ${ }^{52,53}$, as was the case in our patients.

\section{Study limitations}

This study also has some limitations. First, the follow-up time was relatively short. Given the median follow-up (30 months) time and the mean time of clinical failure (35 months), we acknowledge that longer follow-up is necessary to draw definite conclusions. The second limitation is inherent to a retrospective study, which carries the risk of selection bias. 


\section{Subtotal resection followed by GKS versus primary GKS in large VS}

After separately analyzing both treatment strategies of subtotal resection followed by $\mathrm{GKS}^{32}$ versus primary GKS for large VSs, we performed a matched-cohort analysis, in which the 2 groups were matched for age and VS volume. Of course, after matching, one still deals with confounding by indication. A hazard ratio $(\mathrm{HR})$ of $2.5(\mathrm{P}=0.17)$ was found for clinical failure, when performing primary GKS. HR for radiological failure was $1(P=0.94)$ and so there was no difference between the 2 treatment strategies in terms of radiological growth control. The following conclusion can be drawn when choosing between subtotal resection followed by GKS or primary GKS for large VSs: primary GKS leads to more instances of clinical failure (in part due to the diminished reserve to watch tumor growth or transient swelling) after treatment. Therefore, subtotal resection followed by GKS seems a more secure option than primary GKS for large VSs. In case of a patient's wishes, in the presence of comorbidity or functional hearing, for a VS smaller than $4 \mathrm{~cm}$ in the extracanalicular dimension, and in the absence of symptomatic mass effect, primary GKS is also a valid treatment option.

\section{Conclusion}

Primary GKS in large VS leads to acceptable growth and clinical control rates, with the chance of hearing preservation. Although we noted a higher incidence of clinical control failure and postradiosurgical morbidity as compared with results published for smaller VSs, primary radiosurgery is suitable for a selected group of patients, especially in those who have serviceable hearing or are unable to undergo surgery because of comorbidity. The absence of symptomatology due to mass effect is essential, as are close clinical and radiological follow-ups, because there is little reserve to observe tumor growth or swelling. In case of treatment failure, a second GKS or microsurgery is possible. 


\section{References}

1. Yu CP, Cheung JY, Leung S, Ho R. Sequential volume mapping for confirmation of negative growth in vestibular schwannomas treated by gamma knife radiosurgery. J Neurosurg. 2000;93 Suppl 3:82-9.

2. Prasad D, Steiner M, Steiner L. Gamma surgery for vestibular schwannoma. J Neurosurg. 2000;92:745-59.

3. Pollock BE, Lunsford LD, Flickinger JC, Clyde BL, Kondziolka D. Vestibular schwannoma management. Part I. Failed microsurgery and the role of delayed stereotactic radiosurgery. J Neurosurg. 1998;89:944-8.

4. Pollock BE, Link MJ. Vestibular schwannoma radiosurgery after previous surgical resection or stereotactic radiosurgery. Prog Neurol Surg. 2008;21:163-8.

5. Pollock BE. Vestibular schwannoma management: an evidence-based comparison of stereotactic radiosurgery and microsurgical resection. Prog Neurol Surg. 2008;21:222-7.

6. Lunsford LD, Niranjan A, Flickinger JC, Maitz A, Kondziolka D. Radiosurgery of vestibular schwannomas: summary of experience in 829 cases. J Neurosurg. 2005;102 Suppl:195-9.

7. Hasegawa T, Fujitani S, Katsumata S, Kida Y, Yoshimoto M, Koike J. Stereotactic radiosurgery for vestibular schwannomas: analysis of 317 patients followed more than 5 years. Neurosurgery. 2005;57:257-65; discussion -65.

8. Friedman WA, Bradshaw P, Myers A, Bova FJ. Linear accelerator radiosurgery for vestibular schwannomas. J Neurosurg. 2006;105:657-61.

9. Chung WY, Liu KD, Shiau CY, Wu HM, Wang LW, Guo WY, Ho DM, Pan DH. Gamma knife surgery for vestibular schwannoma: 10-year experience of 195 cases. J Neurosurg. 2005;102 Suppl:87-96.

10. Briggs RJ, Luxford WM, Atkins JS, Jr., Hitselberger WE. Translabyrinthine removal of large acoustic neuromas. Neurosurgery. 1994;34:785-90; discussion 90-1.

11. Grey PL, Moffat DA, Palmer CR, Hardy DG, Baguley DM. Factors which influence the facial nerve outcome in vestibular schwannoma surgery. Clin Otolaryngol Allied Sci. 1996;21:409-13.

12. Jung S, Kang SS, Kim TS, Kim HJ, Jeong SK, Kim SC, Lee JK, Kim JH, Kim SH, Lee $\mathrm{JH}$. Current surgical results of retrosigmoid approach in extralarge vestibular schwannomas. Surg Neurol. 2000;53:370-7; discussion 7-8.

13. Lanman TH, Brackmann DE, Hitselberger WE, Subin B. Report of 190 consecutive cases of large acoustic tumors (vestibular schwannoma) removed via the translabyrinthine approach. J Neurosurg. 1999;90:617-23.

14. Mamikoglu B, Wiet RJ, Esquivel CR. Translabyrinthine approach for the management of large and giant vestibular schwannomas. Otol Neurotol. 2002;23:224-7.

15. Samii M, Gerganov VM, Samii A. Functional outcome after complete surgical removal of giant vestibular schwannomas. J Neurosurg. 2010;112:860-7.

16. Wiet RJ, Mamikoglu B, Odom L, Hoistad DL. Long-term results of the first 500 cases of acoustic neuroma surgery. Otolaryngol Head Neck Surg. 2001;124:64551.

17. Zhang X, Fei Z, Chen YJ, Fu LA, Zhang JN, Liu WP, He XS, Jiang XF. Facial nerve function after excision of large acoustic neuromas via the suboccipital retrosigmoid approach. J Clin Neurosci. 2005;12:405-8. 
18. Samii M, Matthies C. Management of 1000 vestibular schwannomas (acoustic neuromas): surgical management and results with an emphasis on complications and how to avoid them. Neurosurgery. 1997;40:11-21; discussion -3.

19. Bennett M, Haynes DS. Surgical approaches and complications in the removal of vestibular schwannomas. Otolaryngol Clin North Am. 2007;40:589-609, ix-x.

20. Inoue HK. Low-dose radiosurgery for large vestibular schwannomas: long-term results of functional preservation. J Neurosurg. 2005;102 Suppl:111-3.

21. Lederman G, Lowry J, Wertheim S, Fine M, Lombardi E, Wronski M, Arbit E. Acoustic neuroma: potential benefits of fractionated stereotactic radiosurgery. Stereotactic and functional neurosurgery. 1997;69(1-4 Pt 2):175-82.

22. Litvack ZN, Noren G, Chougule PB, Zheng Z. Preservation of functional hearing after gamma knife surgery for vestibular schwannoma. Neurosurg Focus. 2003;14:e3.

23. Samii M, Gerganov V, Samii A. Improved preservation of hearing and facial nerve function in vestibular schwannoma surgery via the retrosigmoid approach in a series of 200 patients. J Neurosurg. 2006;105:527-35.

24. Post KD, Eisenberg MB, Catalano PJ. Hearing preservation in vestibular schwannoma surgery: what factors influence outcome? J Neurosurg. 1995;83:1916.

25. Hecht CS, Honrubia VF, Wiet RJ, Sims HS. Hearing preservation after acoustic neuroma resection with tumor size used as a clinical prognosticator. Laryngoscope. 1997; 107:1122-6.

26. Fischer G, Fischer C, Remond J. Hearing preservation in acoustic neurinoma surgery. J Neurosurg. 1992;76:910-7.

27. Rowe JG, Radatz MW, Walton L, Hampshire A, Seaman S, Kemeny AA. Gamma knife stereotactic radiosurgery for unilateral acoustic neuromas. J Neurol Neurosurg Psychiatry. 2003;74:1536-42.

28. Mandl ES, Meijer OW, Slotman BJ, Vandertop WP, Peerdeman SM. Stereotactic radiation therapy for large vestibular schwannomas. Radiother Oncol. 2010;95:948.

29. Yang HC, Kano H, Awan NR, Lunsford LD, Niranjan A, Flickinger JC, Novotny J, Bhatnagar JP, Kondziolka D. Gamma Knife radiosurgery for larger-volume vestibular schwannomas. J Neurosurg. 2011;114:801-7.

30. van de Langenberg R, de Bondt BJ, Nelemans PJ, Baumert BG, Stokroos RJ. Follow-up assessment of vestibular schwannomas: volume quantification versus two-dimensional measurements. Neuroradiology. 2009;51:517-24.

31. Koos WT, Day JD, Matula C, Levy DI. Neurotopographic considerations in the microsurgical treatment of small acoustic neurinomas. J Neurosurg. 1998;88:50612.

32. van de Langenberg R, Hanssens PE, van Overbeeke JJ, Verheul JB, Nelemans PJ, de Bondt BJ, Stokroos RJ. Management of large vestibular schwannoma. Part I. Planned subtotal resection followed by Gamma Knife surgery: radiological and clinical aspects. J Neurosurg. 2011; 115:875-84.

33. Committee on Hearing and Equilibrium guidelines for the evaluation of hearing preservation in acoustic neuroma (vestibular schwannoma). American Academy of Otolaryngology-Head and Neck Surgery Foundation, INC. Otolaryngol Head Neck Surg. 1995;113:179-80.

34. House JW, Brackmann DE. Facial nerve grading system. Otolaryngol Head Neck Surg. 1985;93:146-7. 
35. Iwai $\mathrm{Y}$, Yamanaka $\mathrm{K}$, Ishiguro $\mathrm{T}$. Surgery combined with radiosurgery of large acoustic neuromas. Surg Neurol. 2003;59:283-9; discussion 9-91.

36. Linskey ME, Lunsford LD, Flickinger JC. Neuroimaging of acoustic nerve sheath tumors after stereotaxic radiosurgery. Ajnr. 1991;12:1165-75.

37. Wandong S, Meng L, Xingang L, Yuguang L, Shugan Z, Lei W, Chengyuan W. Cystic acoustic neuroma. J Clin Neurosci. 2005;12:253-5.

38. Pendl G, Ganz JC, Kitz K, Eustacchio S. Acoustic neurinomas with macrocysts treated with Gamma Knife radiosurgery. Stereotactic and functional neurosurgery. 1996;66 Suppl 1:103-11.

39. Charabi S, Klinken L, Tos M, Thomsen J. Histopathology and growth pattern of cystic acoustic neuromas. Laryngoscope. 1994;104:1348-52.

40. Okunaga T, Matsuo T, Hayashi N, Hayashi Y, Shabani HK, Kaminogo M, Ochi M, Nagata I. Linear accelerator radiosurgery for vestibular schwannoma: measuring tumor volume changes on serial three-dimensional spoiled gradient-echo magnetic resonance images. J Neurosurg. 2005;103:53-8.

41. Flickinger JC, Kondziolka D, Niranjan A, Maitz A, Voynov G, Lunsford LD. Acoustic neuroma radiosurgery with marginal tumor doses of 12 to $13 \mathrm{~Gy}$. Int J Radiat Oncol Biol Phys. 2004;60:225-30.

42. Ito K, Shin M, Matsuzaki M, Sugasawa K, Sasaki T. Risk factors for neurological complications after acoustic neurinoma radiosurgery: refinement from further experiences. Int J Radiat Oncol Biol Phys. 2000;48:75-80.

43. Williams JA. Fractionated stereotactic radiotherapy for acoustic neuromas: preservation of function versus size. J Clin Neurosci. 2003;10:48-52.

44. Fuentes S, Arkha Y, Pech-Gourg G, Grisoli F, Dufour H, Regis J. Management of large vestibular schwannomas by combined surgical resection and gamma knife radiosurgery. Prog Neurol Surg. 2008;21:79-82.

45. Park CK, Jung HW, Kim JE, Son YJ, Paek SH, Kim DG. Therapeutic strategy for large vestibular schwannomas. J Neurooncol. 2006;77:167-71.

46. Yang SY, Kim DG, Chung HT, Park SH, Paek SH, Jung HW. Evaluation of tumour response after gamma knife radiosurgery for residual vestibular schwannomas based on MRI morphological features. J Neurol Neurosurg Psychiatry. 2008;79: 431-6.

47. Thomas C, Di Maio S, Ma R, Vollans E, Chu C, Clark B, Lee R, McKenzie M, Martin M, Toyota B. Hearing preservation following fractionated stereotactic radiotherapy for vestibular schwannomas: prognostic implications of cochlear dose. J Neurosurg. 2007;107:917-26.

48. Timmer FC, Hanssens PE, van Haren AE, Mulder JJ, Cremers CW, Beynon AJ, van Overbeeke JJ, Graamans K. Gamma knife radiosurgery for vestibular schwannomas: results of hearing preservation in relation to the cochlear radiation dose. Laryngoscope. 2009;119:1076-81.

49. Roche PH, Khalil M, Thomassin JM, Delsanti C, Regis J. Surgical removal of vestibular schwannoma after failed gamma knife radiosurgery. Prog Neurol Surg. 2008;21:152-7.

50. Pollock BE, Lunsford LD, Kondziolka D, Sekula R, Subach BR, Foote RL, Flickinger JC. Vestibular schwannoma management. Part II. Failed radiosurgery and the role of delayed microsurgery. J Neurosurg. 1998;89:949-55.

51. Slattery $\mathrm{WH}, 3 \mathrm{rd}$. Microsurgery after radiosurgery or radiotherapy for vestibular schwannomas. Otolaryngol Clin North Am. 2009;42:707-15. 
Chapter 6

52. Yomo S, Arkha Y, Delsanti C, Roche PH, Thomassin JM, Regis J. Repeat gamma knife surgery for regrowth of vestibular schwannomas. Neurosurgery. 2009;64:4854; discussion -5 .

53. Liscak R, Vladyka V, Urgosik D, Simonova G, Vymazal J. Repeated treatment of vestibular schwannomas after gamma knife radiosurgery. Acta Neurochir (Wien). 2009;151:317-24; discussion 24. 


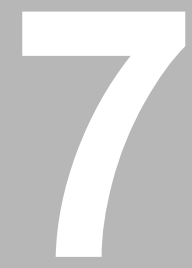

\section{EDITORIAL}

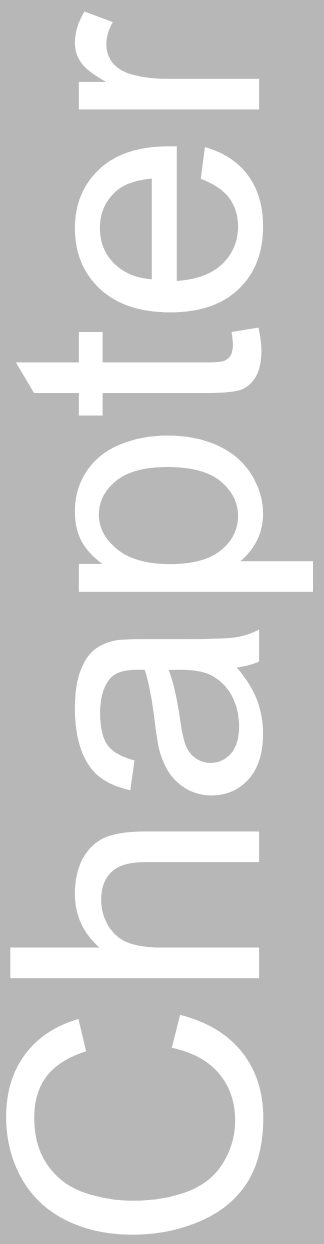

\section{LARGE VESTIBULAR SCHWANNOMAS}

2. ROLE OF RADIOSURGERY FOR LARGER VESTIBULAR SCHWANNOMAS

3. RESPONSE TO EDITORIALS. VESTIBULAR SCHWANNOMAS

1. Nicholas C Bambakidis Simon S Lo Warren R Selman

2. Douglas Kondziolka

3. Rick van de Langenberg Patrick EJ Hanssens Jacobus J van Overbeeke Jeroen B Verheul Patty J Nelemans Bert J de Bondt Robert J Stokroos

1. Journal of Neurosurgery. 2011;115:894-5

2. Journal of Neurosurgery. 2011;115:896-7

3. Journal of Neurosurgery. 2011;115:898-9 
Chapter 7 


\section{Large vestibular schwannomas}

Controversies exist as to what is the best management strategy for vestibular schwannoma (VS). Treatment options include watchful waiting, microsurgery, stereotactic radiosurgery (SRS), and fractionated stereotactic radiotherapy $(\text { FSRT })^{1}$. Microsurgery yields local control similar to that accomplished with $\mathrm{SRS}^{2,3}$. Stereotactic radiosurgery represents an excellent treatment option for patients who are not candidates for microsurgery as a result of comorbidities and for those who refuse to undergo surgery. However, in patients with large VSs, especially those with tumor compressing the brainstem, microsurgery is favored over SRS because the latter procedure is unlikely to lead to the immediate relief of mass effect on the brainstem, and because the risk of brainstem injury from SRS is anticipated to be higher as a result of including a larger volume of brainstem in the high-dose zone. Furthermore, there may be a higher risk of hydrocephalus caused by swelling of the large VS after SRS. Clearly, challenges remain when treating large tumors microsurgically. Although nonaudiofacial complications, such as CSF leakage or wound infection, may occur at consistent rates regardless of tumor size, the rates of permanent hearing loss and postoperative facial nerve weakness are greater when larger tumors are resected ${ }^{4}$. In fact, for tumors larger than $4 \mathrm{~cm}$ in diameter, published rates of long-term good facial nerve function (HouseBrackmann Grade I/II) decrease significantly, approaching $50 \%$ or less in the setting of gross-total resection (GTR) $)^{5,6}$.

In this issue of the Journal of Neurosurgery, van de Langenberg and colleagues $^{7}$ report the treatment outcomes of 50 consecutive patients with large VSs (43 with Koos Grade IV lesions) treated with microsurgery aimed at brainstem decompression, followed by planned postoperative SRS. In a second article, ${ }^{8}$ they report the outcomes for 33 patients with large VSs measuring $4 \mathrm{~cm}$ or less in the maximum dimension, without symptomatic mass effect, and treated with SRS alone. In a study of microsurgery followed by planned SRS, the mean tumor volume reduction after microsurgery was $74.6 \%{ }^{7}$. At a median follow-up of 33.8 months, the crude local control rate was $90 \%$, similar to rates reported in other SRS series ${ }^{1-3,9}$. The rate of facial nerve function preservation (94\%) was also comparable with rates reported in other SRS series ${ }^{1-3,9}$. Significantly, van de Langenberg and colleagues note that the preoperative goal in every case was to attain a tumor size amenable to subsequent SRS treatment once brainstem decompression was achieved. At no time were the authors attempting to achieve GTR, and factors, such as tumor consistency, vascularity, or adherence to the adjacent neurovascular structures, did not alter their treatment planning. Although the effort to minimize audiofacial complications is commendable, the authors may be ignoring an important subset of patients in whom GTR can be safely achieved with 
excellent functional results, that is, younger patients in whom the long-term risk of tumor recurrence following subtotal resection even with SRS is unclear. In such cases, in our opinion, it is still best to begin with a surgical plan consistent with complete resection and to be guided by intraoperative findings that may alter this goal in some circumstances.

In their second study, primary Gamma Knife-based SRS was associated with a local control rate of $88 \%$ and with hearing, facial nerve, and trigeminal preservation rates of $58 \%, 91 \%$, and $86 \%$, respectively ${ }^{8}$. These outcomes were slightly worse than those documented in other SRS series, although these other studies included mostly smaller tumors ${ }^{1-3,9}$. Two patients (6\%) demonstrated hydrocephalus even in the absence of tumor enlargement.

With regard to the SRS dose parameters for the two studies, it appears that the tumor margin dose, ranging from 9.4 to 11.9 Gy and from 10.3 to $13 \mathrm{~Gy}$ in their studies, was slightly lower than the standard margin dose of 12-13 Gy. Furthermore, the authors required only $90 \%$ of the VS to be covered by the prescribed dose of 12-13 Gy. This requirement may account for the lower local control rates of $88 \%$ to $90 \%$, which are at the low end of the reported local control rates of other SRS series. In the SRS treatment of VS, it is important to respect the tolerance of the brainstem. According to the recent Quantitative Analysis of Normal Tissue Effects in the Clinic (QUANTEC) analysis based on SRS data, the brainstem is thought to be able to tolerate a maximum brainstem dose of $12.5 \mathrm{~Gy}$, which is the dose required to permanently control $\mathrm{VS}^{10}$. With meticulous planning, it is possible to generate a plan with $100 \%$ of the tumor receiving the prescribed dose of 12 Gy while keeping the maximum point dose to the brainstem below $12 \mathrm{~Gy}$, further improving the local tumor control rate and keeping the risk of brainstem injury low. In our SRS experience, in which a maximum brainstem point dose of $12 \mathrm{~Gy}$ is allowed, we have not observed any significant complications.

One option that has not been considered by the authors is FSRT. In a large study from the University of Heidelberg featuring 106 patients with VS and a median follow- up of $\mathbf{4 8 . 5}$ months, the actuarial local tumor control rates at 3 and 5 years after FSRT were $94.3 \%$ and $93 \%$, respectively, and the actuarial useful hearing preservation rate was $94 \%$ at 5 years ${ }^{11}$. The rate of radiationinduced toxicity to the trigeminal and facial nerves was $3.4 \%$ and $2.3 \%$, respectively. Fractionated stereotactic radiotherapy is certainly a viable treatment option for patients deemed unsuitable for microsurgery or SRS. In general, patients with larger tumors (>3-4 cm in size) may be better suited for FSRT if microsurgery is not feasible or is refused by patients.

To optimize outcomes, the importance of close collaboration among neurosurgeons, otolaryngologists, and radiation oncologists in the care of patients with VS cannot be overemphasized. It is imperative that patients with 
VS undergo interdisciplinary evaluation before a final decision is made regarding the best treatment option. 


\section{References}

1. Kondziolka D, Lunsford LD, Flickinger JC. Comparison of management options for patients with acoustic neuromas. Neurosurg Focus. 2003;14:e1.

2. Pollock BE, Driscoll CL, Foote RL, Link MJ, Gorman DA, Bauch CD, Mandrekar $\mathrm{JN}$, Krecke $\mathrm{KN}$, Johnson $\mathrm{CH}$. Patient outcomes after vestibular schwannoma management: a prospective comparison of microsurgical resection and stereotactic radiosurgery. Neurosurgery. 2006;59:77-85; discussion 77-85.

3. Regis J, Pellet W, Delsanti C, Dufour H, Roche PH, Thomassin JM, Zanaret M, Peragut JC. Functional outcome after gamma knife surgery or microsurgery for vestibular schwannomas. J Neurosurg. 2002;97:1091-100.

4. Sughrue ME, Yang I, Aranda D, Rutkowski MJ, Fang S, Cheung SW, Parsa AT. Beyond audiofacial morbidity after vestibular schwannoma surgery. J Neurosurg. 2011;114:367-74.

5. Mamikoglu B, Wiet RJ, Esquivel CR. Translabyrinthine approach for the management of large and giant vestibular schwannomas. Otol Neurotol. 2002;23:224-7.

6. Samii M, Gerganov VM, Samii A. Functional outcome after complete surgical removal of giant vestibular schwannomas. J Neurosurg. 2010;112:860-7.

7. van de Langenberg R, Hanssens PE, van Overbeeke JJ, Verheul JB, Nelemans PJ, de Bondt BJ, Stokroos RJ. Management of large vestibular schwannoma. Part I. Planned subtotal resection followed by Gamma Knife surgery: radiological and clinical aspects. J Neurosurg. 2011;115:875-84.

8. van de Langenberg R, Hanssens PE, Verheul JB, van Overbeeke JJ, Nelemans PJ, Dohmen AJ, de Bondt BJ, Stokroos RJ. Management of large vestibular schwannoma. Part II. Primary Gamma Knife surgery: radiological and clinical aspects. J Neurosurg. 2011;115:885-93.

9. Yang HC, Kano H, Awan NR, Lunsford LD, Niranjan A, Flickinger JC, Novotny J, Jr., Bhatnagar JP, Kondziolka D. Gamma Knife radiosurgery for larger-volume vestibular schwannomas. Clinical article. J Neurosurg. 2011;114:801-7.

10. Mayo C, Yorke E, Merchant TE. Radiation associated brainstem injury. Int J Radiat Oncol Biol Phys. 2010;76(3 Suppl):S36-41.

11. Combs SE, Volk S, Schulz-Ertner D, Huber PE, Thilmann C, Debus J. Management of acoustic neuromas with fractionated stereotactic radiotherapy (FSRT): long-term results in 106 patients treated in a single institution. Int J Radiat Oncol Biol Phys. 2005;63:75-81. 


\section{Role of radiosurgery for larger vestibular schwannomas}

The authors from Maastricht University in The Netherlands discuss the roles of resection and Gamma Knife surgery (GKS) in the treatment of patients with larger volume vestibular schwannomas (VSs). In Part I, they discuss the indications for and outcomes in patients who underwent planned subtotal resection followed by $\mathrm{GKS}^{1}$. In Part II, they discuss the outcomes in patients who underwent primary GKS alone ${ }^{2}$. The editorial board of the Journal of Neurosurgery had numerous questions for the authors during the review process of these reports, and many focused on the indications for one approach or another, both from the perspective of patient choice and medical issues. The factors of age, suitability for resection, degree of disability, degree of brainstem compression, and symptoms related to mass effect were important, as the authors note. Perhaps most important in their practice was simply patient choice.

When resection followed by radiosurgery was used, their philosophy was to try to reduce the risk of facial nerve injury by leaving tumor along the facial nerve and performing radiosurgery on any residual tumor once the patient had recovered from the resection. The surgery was performed via the translabyrinthine (25 patients) or retrosigmoid (25 patients) approach. Facial nerve function was good (House-Brackmann Grade I or II in $94 \%$ of patients), and indeed 1 of 2 patients who underwent treatment to attempt hearing preservation did maintain serviceable hearing after combined resection and radiosurgery.

In the group of 33 patients who underwent GKS alone, one questions the definition of the term "large." The authors define it as a tumor "at least indenting the brainstem" and whose volume is over $6 \mathrm{~cm}^{3}$. Most centers consider GKS for patients with small or medium-sized VSs. Most can agree on the definition of "small." "Medium" is a little less clear, however; at our own center, it typically represents lesions with a maximum extracanalicular diameter $<3 \mathrm{~cm}$. The majority of tumors larger than $2 \mathrm{~cm}$ in the extracanalicular extent do indeed indent the brainstem, but I would not call these lesions "large." In fact, tumors that indent the middle cerebellar peduncle are common to radiosurgery. Typically, they take many years to grow into the brainstem, and many eventually regress away from the brainstem over time.

Recently, we described our own series of lesions with an extracanalicular extent over $3 \mathrm{~cm}$, calling them "larger." We found that the need for subsequent resection was greater than for the smaller tumors ( $9 \%$ compared with $2 \%$ ). On the other hand, approximately $90 \%$ of patients who chose radiosurgery for tumors between 3 and $4 \mathrm{~cm}$ were satisfied with their outcomes. We did not perform radiosurgery for tumors larger than $4 \mathrm{~cm}$ in the maximum extracanalicular diameter ${ }^{3}$. 
Earlier today, by chance, I gave a lecture on VS management, including the indications for our different options, to a group of neurosurgeons, radiation oncologists, and otologists. At our center, major indications for resection are disabling ataxia, intractable headache, hydrocephalus, medically refractory trigeminal neuralgia, and an unclear diagnosis. If these criteria are not present, we think that radiosurgery is a viable option. Indeed, four level-2 matchedcohort studies $^{4-7}$ indicate that GKS outcomes were either better than or similar to those for resection, depending on the outcome variable. Indeed, in no instance was resection a better choice, even when one considered longer-term tumor control. Nevertheless, many patients continue to choose resection. Those that do so make their choice based on their own personal preference regardless of the literature. This is not to suggest that they are wrong but only to say that they continue to make that particular choice. Perhaps they hope to avoid serial long-term follow-up or targeted radiation exposure, and so they accept the risks of open surgery. Perhaps they are simply comfortable in the hands of their neurosurgeon and otologist.

The Maastricht group makes a number of conclusions based on their 2 reports. In Part I, they conclude that based on the growth control and facial nerve function results as well as the possibility for hearing preservation and the reduced complication rate, subtotal resection followed by GKS can be the treatment option of choice in patients with large VSs. In Part II, they note a higher rate of postradiosurgical morbidity in comparison with the results published for smaller-volume tumors. They still believe that primary radiosurgery is suitable for a group of patients who are unable to undergo surgery because of a comorbidity or the absence of disabling symptoms. Both conclusions are reasonable. Their reports indicate a growing willingness to "push the borders" of radiosurgery to tumors with larger volumes. For smaller tumors, the data are clear, long term, and consistent. The value of radiosurgery in selected patients with large tumors is encouraging but must be individualized based on the experience of the surgeon and the expectations of the patient. 


\section{References}

1. van de Langenberg R, Hanssens PE, van Overbeeke JJ, Verheul JB, Nelemans PJ, de Bondt BJ, Stokroos RJ. Management of large vestibular schwannoma. Part I. Planned subtotal resection followed by Gamma Knife surgery: radiological and clinical aspects. J Neurosurg. 2011;115:875-84.

2. van de Langenberg R, Hanssens PE, Verheul JB, van Overbeeke JJ, Nelemans PJ, Dohmen AJ, de Bondt BJ, Stokroos RJ. Management of large vestibular schwannoma. Part II. Primary Gamma Knife surgery: radiological and clinical aspects. J Neurosurg. 2011;115:885-93.

3. Yang HC, Kano H, Awan NR, Lunsford LD, Niranjan A, Flickinger JC, Novotny J, Jr., Bhatnagar JP, Kondziolka D. Gamma Knife radiosurgery for larger-volume vestibular schwannomas. Clinical article. J Neurosurg. 2011;114:801-7.

4. Myrseth E, Moller P, Pedersen PH, Lund-Johansen M. Vestibular schwannoma: surgery or gamma knife radiosurgery? A prospective, nonrandomized study. Neurosurgery. 2009;64:654-61; discussion 61-3.

5. Pollock BE, Driscoll CL, Foote RL, Link MJ, Gorman DA, Bauch CD, Mandrekar $\mathrm{JN}$, Krecke $\mathrm{KN}$, Johnson $\mathrm{CH}$. Patient outcomes after vestibular schwannoma management: a prospective comparison of microsurgical resection and stereotactic radiosurgery. Neurosurgery. 2006;59:77-85; discussion 77-85.

6. Pollock BE, Lunsford LD, Kondziolka D, Flickinger JC, Bissonette DJ, Kelsey SF, Jannetta PJ. Outcome analysis of acoustic neuroma management: a comparison of microsurgery and stereotactic radiosurgery. Neurosurgery. 1995;36:215-24; discussion 24-9.

7. Regis J, Pellet W, Delsanti C, Dufour H, Roche PH, Thomassin JM, Zanaret M, Peragut JC. Functional outcome after gamma knife surgery or microsurgery for vestibular schwannomas. J Neurosurg. 2002;97:1091-100. 


\section{Response to editorials. Vestibular schwannomas}

We appreciate the valuable editorial comments given by these experts in the field. They draw attention to some important questions encountered in the management of vestibular schwannoma (VS) and underline the change in our treatment strategy over the recent years to today, in particular, to the issue addressed by Dr. Kondziolka, that is, to "push the borders" of primary Gamma Knife surgery (GKS) for large VSs. This impulse concords with what we have been experiencing in recent years, encouraged by the initial results of GKS in patients with a large VS who could not undergo open surgery. Just as the borders are shifting, so too are the indications. It is therefore quite right that numerous questions were asked by the editorial board regarding the indications for one treatment or another in these 2 retrospective studies ${ }^{1,2}$. Because indications are evolving, we do not have all the answers. Some of the aforementioned factors, such as age, degree of brainstem compression, and degree of disability, are debatable indications for resection. Others, such as symptoms of mass effect, are less debatable, in our opinion. Indeed, as indications are not clear-cut, the choice of the patient is important in our practice.

Regarding the second paper, $^{2}$ Dr. Kondziolka argues that the treated VS should not be qualified as large. We agree that our definition of "large" (volume $>6 \mathrm{~cm}^{3}$ ) is arbitrary, and this volumetric cutoff point has not been used before in the literature. However, all VSs mentioned in the present study were at least Koos Grade III. Extracanalicular dimensions ranged from 2.1 to $4.0 \mathrm{~cm}$, and all tumors indented the brainstem. Perhaps a better definition of VS in the primary GKS study would be, as suggested, "larger VS."

It is interesting to see a higher number of reinterventions in primary GKS for larger VSs - both in the Pittsburgh study ${ }^{3}$ and in the present study ${ }^{2}$ - than after primary GKS for small/medium-sized VSs. In our opinion this reflects the tendency to intervene more aggressively in cases of transient swelling of larger VSs as compared with smaller ones, rather than any intrinsic tumor factors or differences in dosimetry.

The editorial from our colleagues at University Hospital Case Center, Cleveland, illustrates the continuing debate about the best microsurgical treatment for large VSs. They mention that one should try to achieve grosstotal resection. The extent of resection can be diminished depending on the intraoperative findings. In our experience any attempt to dissect the facial nerve and completely resect a large VS substantially increases the risk of facial nerve dysfunction. Therefore, we do not routinely start with the intention to perform a gross-total resection. Obviously, it appears that we tend to "stay on the safe side" as compared with our colleagues from University Hospital Case Center. 
This approach not only reflects a different philosophy, but also may indicate a difference in experience and surgical skills ${ }^{1}$.

The same group mentions that the $94 \%$ facial nerve preservation rate in our first study ${ }^{1}$ resembles the outcome in other SRS studies. However, in the cited studies, patients had not first been treated with subtotal resection ${ }^{3-6}$, precluding comparisons. Furthermore, they suggest that the local control rate in our 2 studies seems lower than rates reported in the literature. A possible explanation they mention is that our dose is slightly lower than doses prescribed elsewhere. We attribute our local control rate mainly to the use of volume measurements, which are more sensitive for detecting growth as compared with 2D measurements ${ }^{7}$. The way in which we prescribed the dose, that is, $12-13$ Gy to $90 \%$ of the tumor volume, resulted in a lower tumor margin dose than in other series. To the best of our knowledge, there are no doseescalation studies documenting a dose response for tumor control with a cutoff of 12-13 Gy for the tumor margin dose. Our policy on dose prescription was driven by an attempt to further reduce toxicity.

The Cleveland group also suggested using fractionated radiotherapy (FRT). This might be a better therapeutic strategy for large VSs; however, the cited article does not specifically focus on large VS but instead reports outcomes after FRT, $50 \%$ of which are small/medium VSs ${ }^{8}$. Clearly, at the GammaKnife Center Tilburg, FRT cannot be performed. A randomized trial comparing FRT versus GKS for large VS could provide more insight into these specific cases but would be very difficult to conduct. We fully agree with the Cleveland group that interdisciplinary evaluation of our patients is mandatory and the cornerstone of VS treatment. 


\section{References}

1. van de Langenberg $R$, Hanssens PE, van Overbeeke JJ, Verheul JB, Nelemans PJ, de Bondt BJ, Stokroos RJ. Management of large vestibular schwannoma. Part I. Planned subtotal resection followed by Gamma Knife surgery: radiological and clinical aspects. J Neurosurg. 2011;115:875-84.

2. van de Langenberg R, Hanssens PE, Verheul JB, van Overbeeke JJ, Nelemans PJ, Dohmen AJ, de Bondt BJ, Stokroos RJ. Management of large vestibular schwannoma. Part II. Primary Gamma Knife surgery: radiological and clinical aspects. J Neurosurg. 2011;115:885-93.

3. Yang HC, Kano H, Awan NR, Lunsford LD, Niranjan A, Flickinger JC, Novotny J, Jr., Bhatnagar JP, Kondziolka D. Gamma Knife radiosurgery for larger-volume vestibular schwannomas. Clinical article. J Neurosurg. 2011;114:801-7.

4. Kondziolka D, Lunsford LD, Flickinger JC. Comparison of management options for patients with acoustic neuromas. Neurosurg Focus. 2003;14:e1.

5. Pollock BE, Driscoll CL, Foote RL, Link MJ, Gorman DA, Bauch CD, Mandrekar $\mathrm{JN}$, Krecke $\mathrm{KN}$, Johnson $\mathrm{CH}$. Patient outcomes after vestibular schwannoma management: a prospective comparison of microsurgical resection and stereotactic radiosurgery. Neurosurgery. 2006;59:77-85; discussion 77-85.

6. Regis J, Pellet W, Delsanti C, Dufour H, Roche PH, Thomassin JM, Zanaret M, Peragut JC. Functional outcome after gamma knife surgery or microsurgery for vestibular schwannomas. J Neurosurg. 2002;97:1091-100.

7. van de Langenberg R, de Bondt BJ, Nelemans PJ, Baumert BG, Stokroos RJ. Follow-up assessment of vestibular schwannomas: volume quantification versus two-dimensional measurements. Neuroradiology. 2009;51:517-24.

8. Combs SE, Volk S, Schulz-Ertner D, Huber PE, Thilmann C, Debus J. Management of acoustic neuromas with fractionated stereotactic radiotherapy (FSRT): long-term results in 106 patients treated in a single institution. Int J Radiat Oncol Biol Phys. 2005;63:75-81. 
DISCUSSION AND CONCLUSION

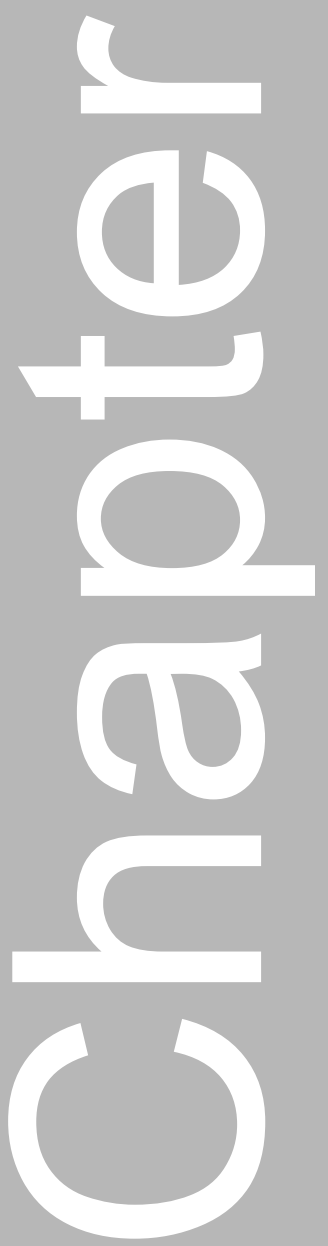


Chapter 8 


\section{Thesis discussion and conclusion}

Management of vestibular schwannomas (VS) is a continuously evolving field: over recent decades, a clear shift can be seen in the management of VS from saving the patient's life, to saving the patient's function. This shift comes with a more conservative approach to these benign neoplasms, in which the wait and scan (W\&S) policy plays a major role. Insight into the growth patterns of VS led to the establishment of this W\&S policy, in which patients are monitored by sequential MRI. Most patients, diagnosed with a VS nowadays, enter the W\&S policy. Patients with a growing small/medium sized VS, who were mainly treated surgically a decade ago, are now treated by means of radiosurgery. Similar outcome rates are reported when comparing both techniques ${ }^{1,2}$. Microsurgical removal, with possible morbidity, for example to the cranial nerves or brain stem, and carrying with it, even in the best microsurgical centers, a mortality rate of $1 \%^{3,4}$, can often be avoided in exchange for a noninvasive radiosurgical treatment.

This thesis describes new developments in the management of VS. The first goal of this thesis was to evaluate the current practice of measuring the VS size and evaluating growth in a W\&S policy, in which the use of the conventional two-dimensional (2D) measurements were compared with the use of volume measurements (Chapter 2). The second goal was to investigate whether or not the use of the more sensitive volume measurements would have implications for the detection of risk factors regarding VS growth or hearing deterioration, knowledge which is currently lacking (Chapter 3 ). The third goal was to use the volume measurements to re-evaluate an established VS treatment technique (LINAC-based stereotactic radiosurgery) (Chapter 4) and to analyze treatment techniques in large VS which are not currently in widespread use, but may more specifically preserve the function of a patient when compared with the established treatment regime in large VS (Chapter 5, 6 and 7$)$.

\section{Evaluation of measuring VS size}

Because many patients enter a W\&S policy when a VS has been diagnosed, an adequate measuring technique to evaluate the VS behavior is essential. So far, sequential MRI is the best way to asses the presence of VS growth. In current clinical practice, these observations are based on 2D measurements, although volume measurements would seem to be more precise because a VS is not a flat object and grows asymmetrically in all directions. Thus, slight volume changes are not detected with 2D measurements. In addition, a certain increase in diameter corresponds to a larger increase in volume in larger VS, because diameters are related to volume by a cubic function. The measuring techniques cannot be verified by a microsurgical resection. In order to 
determine which measuring method is most precise, the best way to compare these measuring modalities is to look at their reproducibility. Reproducibility measures consist of agreement and reliability parameters ${ }^{5,6}$. Agreement parameters measure the ability to achieve the same value in two measurements and give an indication of the size of measurement errors. Reliability parameters assess whether measurements can be used to distinguish patients from each other despite measurement error.

The theoretical notion of the superiority of volume measurements was confirmed in Chapter 2. VS measurements (both 2D and volume measurements) showed the best reproducibility when performed on contrast enhanced T1-weighted images (CE T1-WI), rather than on T2-weighted images (T2-WI). The sharp surrounding of the VS, enhanced by contrast on CE T1-WI, ensured that the VS was better differentiated from surrounding tissue. Contrast enhanced T1-weighted MRI volume measurements showed a better interobserver agreement and reliability, when compared with 2D measurements. When taking the different VS stages into account, the interobserver agreement of $2 \mathrm{D}$ and volume measurements were comparable in intracanalicular VS, making both measuring modalities suitable for these small VS. Larger VS were best measured with volume measurements on CE T1-WI. This difference can be explained by the fact that the measurement error is inversely related to the size of an object that needs to be measured: the smaller the VS, the larger the measurement error. The superiority of volume measurements confirms previous results, although, direct comparison of these studies is hampered because of differences in the volume measuring technique $^{7}$ and slice interval settings ${ }^{8}$. Other publications favoring $2 \mathrm{D}$ measurements above volume measurements, are generally older ${ }^{9-11}$, have used less reliable CT scans ${ }^{11}$, or have not performed a statistically based comparison between the two techniques ${ }^{9,10}$.

Despite evidence of the superiority of volume measurements, 2D measurements are still being used in clinical practice because they can be performed faster and, generally speaking, on all radiological PACS stations (Picture Archiving and Communication System), which are available in all radiology departments, unlike the volume measuring software. For all measuring techniques, it is of major importance to have an idea of "significant growth", which is growth measured beyond the particular measurement method's measurement error. In 2D measurements, clinicians and numerous authors, use a difference of 1 or 2 millimeters between subsequent scans as an arbitrary criterion for significant growth. This has "simply" been stated as the measure for growth, but the true measurement error has not been calculated or published in the literature before. In Chapter 2 this measurement error was calculated for both the 2D and volume measurements. On axial CE T1-WI the measurement error varied between $2.12 \mathrm{~mm}$ for the mediolateral VS dimension 
and $2.98 \mathrm{~mm}$ for the craniocaudal dimension on CE T1-WI. This indicated that there is a measurement error in $2 \mathrm{D}$ measurements, which is not being recognized when a measured increase in size of only 1 or $2 \mathrm{~mm}$ between follow-up (FU) scans is considered as tumor growth. Therefore, treatment decisions should be made with caution if one uses these arbitrary criteria when evaluating VS growth two-dimensionally.

When analyzing studies which report on measurement errors of different measuring techniques, it is important to realize that this error will always be influenced by the quality of the images, the software used, and the experience of the radiologist performing the measurements. Published measurement errors can, therefore, never simply be adopted in a certain protocol for evaluating VS, without taking the techniques of acquiring and measuring images in these studies and in one's own practice into account.

\section{Analysis of risk factors}

The growth rate of a VS is highly variable, with rates of $30-90 \%$ being reported, depending at least in part on the length of the observation period ${ }^{12,13}$ and on the measuring technique used. The most common symptom in patients diagnosed with a VS is sensorineural hearing loss ${ }^{9,14}$. Because containment of growth and maintenance of functional hearing are the major goals in a W\&S policy, many authors have tried to find factors predicting VS growth and hearing deterioration. If risk factors have been found regarding VS growth, they have generally been inconsistent across studies. An important limitation of these studies is that they measured the VS two-dimensionally, with cut-off points for significant growth that lie, according to our results, inside the measurement error. Therefore, we tried to identify risk factors that can be used to highlight patients at the start of a W\&S policy who are at risk of developing significant VS growth or hearing deterioration during FU, by using volume measurements, with the corresponding measurement error. By doing so, we expected to have a more sensitive tool for detecting correlations, if they truly exist. In Chapter 3 , a multivariate analysis illustrated that the evaluation of baseline patient- and VS- characteristics, revealed no factors predicting growth during FU. However, growth within the first year of FU was significantly related to further growth during FU. Regarding preservation of hearing, we found a hypointense labyrinth on T2-weighted MRI at the start of FU to be associated with a significantly worse outcome on the pure tone audiogram (PTA) threshold during FU, when compared with labyrinths with an isointense aspect on T2-WI. This factor could be used to inform the patient about the potentially poorer prognosis of hearing outcome during FU. It might also influence the treating clinician to switch to a hearing preserving treatment in a patient with an absolute wish to preserve hearing (for example, a VS in the only hearing ear). A problem is that, so far, the literature reveals no ideal treatment technique that guarantees long- 
term hearing preservation for small/medium sized VS, as can be read in Chapter 1. The exact cause of the hypointensity on T2-WI is not well understood, but previous studies indicate that the presence of a VS causes a biochemical alteration of the inner ear fluids ${ }^{15-18}$. These alterations may be caused by vascular impairment due to mechanical VS obstruction ${ }^{17,19}$.

Another study finding was that VS patients with a complaint of hearing loss at diagnosis, showed a larger loss in pure tone audiogram (PTA) during FU, when compared with patients without hearing complaints. This means that hearing deterioration is a process that will continue at a faster rate when it has become symptomatic, which is in agreement with the recent findings of Stangerup et al.: established hearing loss is a strong predictor of further hearing loss, whereas patients with minimal PTA losses and a speech discrimination score (SDS) of $100 \%$ are unlikely to lose their functional hearing ${ }^{20}$. No other factors were found to be related to loss of PTA or SDS; hearing loss occurred both in the growing and non-growing group equally. Also, the total VS volume, and the amount of the increase in volume during FU were not related to the amount of hearing loss, or a decrease in hearing over time, respectively.

The present findings lead us to the conclusion that growth occurs irrespective of any factor known at diagnosis, despite the use of the more precise volume measurements. Growth during the first year predicts further growth during FU. This is a useful fact, although one should still bear in mind that VS are known to have a growth pattern which is often variable and growth may still stop after a certain period. Furthermore, the results show that hearing deterioration in VS is not only caused by mechanical compression of the cochlear nerve. Other factors may be involved, for example differences in biological VS behavior, compromise in vascular supply of the cochlear nerve, and biochemical alterations of the inner ear fluid. The latter hypothesis is supported by the findings concerning the labyrinthine hypointensity on T2-WI in Chapter 3.

\section{Evaluation of treatment techniques}

As mentioned above, radiosurgery has replaced microsurgery as the treatment option of choice for most small/medium sized VS. The main goal of a radiosurgical intervention is to arrest VS growth, and therefore lifelong FU remains necessary. In the department of Maastricht Radiation-Oncology (MAASTRO clinic), Maastricht University Medical Center (MUMC), VS patients are treated with linear accelerator (LINAC) based stereotactic radiosurgery or fractionated radiotherapy. Previous studies have described excellent long-term results regarding radiological growth control and absence of additional intervention, with minimal morbidity to the cranial nerves ${ }^{21-24}$. A major disadvantage of these studies is that they have used the suboptimal 2D measurements in order to evaluate the radiological VS control, with measures for significant growth that lie within measurement error. Therefore, these results 
of radiological growth control must be questioned. Only two other known studies have utilized volume measurements to evaluate radiological growth control in VS patients treated with LINAC stereotactic radiosurgery. However, they did not calculate the measurement error for the volume measuring technique they used ${ }^{25,26}$. Chapter 4 described how, in VS LINAC based stereotactic radiosurgery or fractionated radiotherapy, a 4-year probability of radiological growth control can be achieved in $85.4 \%$ of the cases, and a 4 -year probability of absence of additional intervention in $96.4 \%$ of the cases. The radiological control rate matches the results of the two other studies performing volume measurements in VS patient treated with LINAC based radiotherapy: $84.4 \%{ }^{26}$ and $81 \%{ }^{25}$, respectively. The radiological growth control rate is evidently less, when compared to $2 \mathrm{D}$ studies performed in this area, reporting on LINAC or Gamma Knife surgery (GKS). As reported in Chapter 4, median radiological growth control rate in $2 \mathrm{D}$ studies was found to be $96 \%$, while this was $90 \%$ for volume based studies performing radiosurgery for VS. It seems clear that volume measurements are capable of detecting smaller volume changes than can be detected by $2 \mathrm{D}$ measurements. An interesting fact is that, despite the presence of volumetric growth, this did not result in an increased amount of additional intervention (second radiosurgery session or microsurgery). This may be due to the fact that this retrospectively analyzed series was originally measured two-dimensionally in the clinic, thereby leaving some progressing VS unnoticed. This suggestion is supported by the fact that most VS patients classified as "growth", presented with a stable situation after initial growth. The discrepancy between radiological control rate and absence of further intervention also indicates that not all radiological growth is considerable enough for a patient to undergo a second VS related intervention. Transient swelling, a reaction of the VS to the radiotherapy, caused by tumoral edema, was found in $54 \%$ of the patients. In the early years of VS radiotherapy, this was often misunderstood as treatment failure, causing an unnecessary increase in additional treatment. Nowadays the presence of transient swelling is well known; however, it is still difficult to differentiate between swelling and progressive growth during FU in the period after treatment. It seems impossible to create a post-radiotherapeutic time frame to differentiate between transient swelling and growth. Therefore, sequential MRI remains necessary to evaluate the VS behavior after radiotherapy. No risk factors were identified for radiological growth failure or the presence of additional intervention. Previous treatment and stereotactic radiosurgery were associated with a significantly higher chance of transient swelling after treatment.

The fact that a more conservative approach leads to a better preservation of function was explored in Chapters 5 and $\mathbf{6}$. These chapters evaluated the treatment of large VS, a distinct subset of VS. These are VS that compress the 
brain stem or even deviate the $4^{\text {th }}$ ventricle. Especially in the largest, Koos 4 VS, there is no doubt about the need for intervention: waiting is likely to result in further growth, causing possible life-threatening situations. A radiosurgical treatment is not frequently chosen because of the risk of transient swelling. A complete microsurgical resection is believed to be the main treatment option in these situations, however, previous studies evaluating these cases show a suboptimal outcome of the facial nerve, with good function of the facial nerve (House Brackmann I or II) being reported in $27-58 \%$ of the cases ${ }^{27-33}$. In VS microsurgery, facial nerve outcome is inversely related to the VS size ${ }^{30,34}$. This is caused by the fact that the facial nerve is often stretched by, and attached to, the capsule of the VS in larger VS, making it more susceptible to intraoperative injury. A suboptimal facial nerve outcome will severely affect the quality of life of a patient ${ }^{35,36}$. A different option in these patients would be to remove the VS subtotally, leaving VS tissue around the facial nerve, avoiding nerve damage and reducing the VS to a size suitable for radiosurgery. A disadvantage of this method is that regrowth occurs in up to $53 \%$ of cases, of a subtotal resection ${ }^{37-45}$. That regrowth may be prevented by adding GKS to the regimen to gain VS growth control: in small/medium sized VS, excellent results are obtained after GKS treatment ${ }^{46-48}$, similar to LINAC-treated VS. In Chapter 5, patients with a large VS were presented, treated first with a subtotal resection, followed by GKS in the postoperative period. During a median FU of 33.8 months, a radiological growth control of $90 \%$ was seen, and a clinical control rate (absence of further intervention) of $92 \%$. The clinical control rate of these small/medium sized VS remnants is less, when compared with the results obtained in Chapter 4 , in which small/medium sized VS were treated with LINAC-based stereotactic radiosurgery. It is difficult to find an explanation for this difference, especially because similar radiological growth control rates were obtained. In addition, the patients were treated in two different centers (Maastricht and Tilburg) with two different radiosurgical modalities (LINAC and GKS), doubly complicating a clear comparison. A possible explanation for the discrepancy in clinical control may lie in the fact that the mean VS volume after subtotal resection was $3.34 \mathrm{~cm}^{3}$ and the mean volume prior to primary LINAC treatment was $2.09 \mathrm{~cm}^{3}$. However, this difference in volume seems to be too small to explain the discrepancy in clinical control. Another factor may be related to the biological behavior of the former large VS: these large tumors have already proven to have the capacity to show progressive growth, and they may continue to do so, even after a subtotal resection followed by a radiosurgical intervention, being expressed in a diminished clinical control rate in some cases, when compared with small/medium sized VS. If growth was seen in the residual group after GKS, this was generally a progressive phenomenon, in contrary to the small/medium sized group, where we often saw a stable situation after initial growth. 
One year after GKS, facial nerve function was good in $94 \%$ of the patients. In addition, in one of the two patients with serviceable hearing, treated with a retrosigmoid approach, functional hearing was retained until the last FU (37 months after GKS). The facial nerve preserving rate is evidently superior, when compared to results published for complete microsurgical resections in large VS. Moreover, it has been proven that preservation of hearing is not a utopia but a possibility. As yet, however, the numbers ( 1 of the 2 patients) are too low to draw definite conclusions.

There were few complications. It seems that in the present study, fewer complications (CSF leakages, wound infections, meningitis) were encountered, when compared to the literature in which a complete microsurgical resection is described ${ }^{27,29-33}$. This might have been as a result of the diminished operating time and invasiveness of the procedure.

According to these results, the subtotal resection followed by GKS seems a suitable technique for large VS, in order to gain residual VS growth control and to maintain a high chance of preserving facial, or perhaps even cochlear, nerve function. Not everyone agrees with this. In Chapter 7 it was reported that Bambakidis et al. do not agree with the method elaborated in Chapter 5. They suggest that an important subset of patients with a large VS can be safely operated on with a complete resection, with excellent functional results, in contrary to results published in the literature. They especially perform this in younger patients, for whom the long term risk of tumor regrowth after subtotal resection, even with GKS, is unclear. In contrast, the literature shows that in up to $11 \%$ of the patients where a complete VS resection was performed, FU reveals residual or recurrent $\mathrm{VS}^{39,49}$. Bambakidis et al. advise to start with a complete resection, and alter this goal by intraoperative findings. This indicates that there is no communis opinio of how to approach these large VS. In addition to a difference in philosphophy, difference in experience and surgical skill may also play a role.

A second, relatively new treatment option in large VS is primary GKS. As mentioned above, a radiosurgical treatment in large VS is generally not preferable because of the possible risk of transient swelling, with life threatening consequences. Some patients however, present themselves with a large VS without disabling symptoms necessitating surgical intervention. In addition, they may present themselves with serviceable hearing. Some patients may be old or suffer from significant co-morbidity, not making them ideal candidates to undergo major skull base surgery. Moreover, some patients may refuse surgery. The limited results of preserving functional hearing in patients with a large VS after primary GKS show that 41 of the 58 patients reported in literature retained functional hearing ${ }^{50-55}$. When performing a complete microsurgical resection, this ranges from $0-29 \%^{28,29,56-59}$. 
The Gamma Knife Centre, St. Elisabeth Hospital, Tilburg, the Netherlands, started to apply the positive results seen in small/medium sized VS to patients with larger VS who could not undergo an invasive skull base procedure. Relatively good results were seen, when treating these large VS with primary GKS. This knowledge, combined with the encouraging results published in international literature, has made more patients eligible for primary GKS. In Chapter 6, patients with a large VS were suitable candidates for primary GKS if the VS was smaller than $4 \mathrm{~cm}$ in extracanalicular dimension. Larger VS were not selected, because some degree of mass effect, an absolute contraindication for primary GKS, is usually present in larger VS. If patients did not meet these criteria, a subtotal resection followed by GKS was chosen. After a median FU of 30 months, a radiological growth control was seen in $88 \%$ of the patients. A clinical control rate of $79 \%$ was seen in these cases. Of interest here was the greater number of clinical failures in this group, whilst the radiological control rate was comparable with the results obtained in Chapter 5. Although only one patient suffered from severe symptoms after GKS (papilledema, ataxia) necessitating microsurgical intervention, seven patients received additional treatment in this study. The other six patients suffered from mild symptoms (2 patients, facial hypesthesia) or appeared only with asymptomatic growth (4 patients). The problem in large VS treated with primary GKS is that, unlike the situation in small/medium sized VS, there is less reserve to observe postradiosurgical swelling or growth by means of clinical follow up and imaging. In case of symptomatic mass effect (as in one of the cases in our series), it is important to intervene. The same holds true for persisting asymptomatic progression: despite the absence of clinical deterioration, the decision should be made to intervene. The higher risk of additional intervention is an important limitation of primary GKS in large VS and something that should be discussed with the patient prior to treatment.

In the same series, functional hearing was preserved in 58\% during a median FU of 37 months, which is evidently better when compared to results after a complete microsurgical resection of $\mathrm{VS}^{28,29,56-59}$. It seems, therefore, justified to conclude that in patients with a large progressive VS, with functional hearing and a wish to preserve this, primary GKS can be the therapy option of choice, if contraindications for primary GKS are absent. Apart from the one patient developing severe symptomatology, other complications were limited to transient cranial nerve dysfunction ( $9 \%$ facial nerve, $14 \%$ trigeminal nerve) or the need to place a ventriculoperitoneal drainage in symptomatic hydrocephalus (6\%). The results of this study are more reliable compared to other studies focusing on this topic, because other studies did not use volume measurements ${ }^{52-55,60,61}$, included postoperative patients ${ }^{50-55,60,61}$, used CT scans during $\mathrm{FU}^{51,52,55}$ and included patients with neurofibromatosis type $\|^{52,55}$. The encouraging results of this study justify the pushing of the border towards 
tumors of a larger volume undergoing GKS. In Chapter 7, Bambakidis et al. suggested that the use of fractionated radiotherapy (FRT) may be a better option for patients with a large VS. This is a good suggestion, because then the administered radiation is divided over a certain period, instead of delivering the dose at once. However, in a GammaKnife center this is not possible. A randomized trial comparing GKS and FRT could provide more insight in this topic, but would be difficult to conduct.

The chance of receiving additional intervention after primary radiosurgery for small/medium sized VS is very small, as can be read in Chapter 4 . The risk of treatment failure was increased in Chapter 5 and $\mathbf{6}$. As discussed in these chapters, a second radiosurgical procedure is possible in case of treatment failure. This has been proven to be a safe and effective treatment in selected patients ${ }^{62,63}$. If a second radiosurgical procedure is not an option (in the case of a too large or cystic VS, uncontrollable medical symptoms, or the patients wish), salvage surgery can be performed.

The degree of increased microsurgical technical difficulties between nonradiated and radiated tumors has been debated, although agreement regarding the relative difficulty of tumor dissection following radiation therapy has not been reached: some authors have found an increased difficulty ${ }^{67,68}$, whilst others have not ${ }^{64-66}$. Most studies describe an increased percentage of facial nerve morbidity after salvage surgery when compared to non-irradiated $\mathrm{VS}^{66-69}$. However, the goal of these authors was generally to achieve a complete VS resection. This is a more hazardous procedure because of capsular fibrosis and scarring after radiosurgery ${ }^{66,69}$, which are complicating factors additional to the difficulty of dissecting the VS from the facial nerve which is inversely related to VS size ${ }^{30,34}$. Furthermore, after radiosurgery, the nerve's regeneration potential is diminished and recovery from surgical trauma is not as robust ${ }^{69}$.

As mentioned in Chapter 6, our experience is that salvage surgery after failed radiosurgery did not necessarily result in an increased difficulty in resecting a VS. A subtotal resection without facial nerve dissection should be performed in these previously irradiated VS, to maximize patient outcomes ${ }^{64,66,69,70}$, which may then be followed by second radiosurgical treatment of the tumor remnant. Obviously, when discussing a radiosurgical treatment regimen, the possibility of a surgical intervention should be discussed with the patient, including the fact that the salvage surgery may be more difficult. This latter fact, however, does not support a change in the policy of (primary) VS radiosurgery, as elaborated in Chapters 4, 5 and 6.

The treatment options for large VS, as reported in Chapters $\mathbf{5}$ and $\mathbf{6}$, are not used widely at the present time. The introduction of these novel treatment modalities in recent years also means that there are new indicators for such treatments. This was emphasized in Chapter 7, in which it was shown that exact indicators for one specific type of treatment are not as clear cut as they 
are in smaller VS. As such, there is no single best treatment for large VS in general. Several factors should be weighed against each other before a treatment decision is made for each individual patient. Debatable indications for a microsurgical resection are, for example, age, the degree of brain stem compression, and degree of disability. A debatable indication for GKS might be the presence of functional hearing. These indicators represent the gray area, in which indications for a specific treatment option are not clear cut. Symptomatic mass effect is evidently an indication for microsurgery, as is severe comorbidity for GKS. Two other important factors are the patient's expectations and the patient's choice. Also decisive is the experience of the treating (radio) surgeons in dealing with large VS with GKS or microsurgery. The decision for a specific treatment should be made in a multidisciplinary team, in order to further optimize treatment outcomes.

According to the results reported in Chapter $\mathbf{5}$ and $\mathbf{6}$, we can conclude that a subtotal resection followed by GKS is a more secure option than primary GKS, taking the absence of additional intervention into account. In the case of a patient's wish, co-morbidity, or functional hearing, with a VS $<4 \mathrm{~cm}$ in the absence of symptomatic mass effect, primary GKS is also a valid treatment option.

\section{Future perspectives}

Considering the improvements seen in MRI and in specialized software capable of performing volume measurements semi-automatically, it can be expected that the measurement error will decrease in VS measurements performed volumetrically as the human component (the source of substantial measurement error) will diminish. In anticipation of this, it may be expected that volume measurements will replace the "standard" 2D measurements in the FU within the near future, especially for the non- intracanalicular VS. This should apply both to VS being followed in a W\&S policy and to patients being followed after treatment, in order to analyze the treatment effect.

\section{Recommendations for future research}

Longer-term FU studies are necessary in order to confirm the encouraging results obtained so far in the studies which have evaluated subtotal resection followed by GKS and primary GKS in large VS.

A prospective, randomized controlled trial comparing the three established VS treatment modalities, would be of great value, however, as mentioned before, this would be difficult, and in many instances non-ethical to conduct.

A better understanding of the molecular pathobiology of VS may lead to a better understanding of these VS that fail current treatment. In addition, it may lead to novel therapeutics, to augment current modalities of treatment, while further minimizing the morbidity. 


\section{Conclusions}

- Volume measurements are more precise in evaluating VS size than the current 2D measurements that are used in clinical practice. Furthermore, the arbitrary criterion used for $2 \mathrm{D}$ growth (1 or 2 millimeters), lies within measurement error, and should be used with caution.

- In a W\&S policy, growth during the first year predicts further growth during FU, a labyrinthine hypointense signal intensity on T2 weighted MRI and a complaint of hearing loss at presentation predict further hearing loss during FU.

- The radiological growth control rate after LINAC-based stereotactic radiosurgery for VS is lower, when calculated using volume measurements rather than $2 \mathrm{D}$ measurements. This phenomenon is also seen, when evaluating large VS, both those treated with primary GKS and after subtotal resection followed by GKS, and is due to the fact that volume measurements are better able to detect changes in VS size.

- Both a subtotal resection followed by GKS and primary GKS are possible treatment options of choice in large VS, in order have a higher chance of preserving cranial nerve function and to gain VS growth control. However, an increase in clinical failures should be noted when starting with primary GKS in large VS.

- So far, there is no ideal approach for large VS. The treatment should be individualized, based upon symptoms, choice and expectations of the patient and the experience of the skull base team. 


\section{References}

1. Myrseth E, Moller P, Pedersen PH, Lund-Johansen M. Vestibular schwannoma: surgery or gamma knife radiosurgery? A prospective, nonrandomized study. Neurosurgery. 2009;64:654-61; discussion 61-3.

2. Pollock BE, Driscoll CL, Foote RL, Link MJ, Gorman DA, Bauch CD, Mandrekar $\mathrm{JN}$, Krecke $\mathrm{KN}$, Johnson $\mathrm{CH}$. Patient outcomes after vestibular schwannoma management: a prospective comparison of microsurgical resection and stereotactic radiosurgery. Neurosurgery. 2006;59:77-85; discussion 77-85.

3. Samii M, Matthies C. Management of 1000 vestibular schwannomas (acoustic neuromas): surgical management and results with an emphasis on complications and how to avoid them. Neurosurgery. 1997;40:11-21; discussion -3.

4. Bennett M, Haynes DS. Surgical approaches and complications in the removal of vestibular schwannomas. Otolaryngol Clin North Am. 2007;40:589-609, ix-X.

5. de Vet HC, Terwee CB, Knol DL, Bouter LM. When to use agreement versus reliability measures. J Clin Epidemiol. 2006;59:1033-9.

6. Bland JM, Altman DG. Statistical methods for assessing agreement between two methods of clinical measurement. Lancet. 1986;1:307-10.

7. Vokurka EA, Herwadkar A, Thacker NA, Ramsden RT, Jackson A. Using Bayesian tissue classification to improve the accuracy of vestibular schwannoma volume and growth measurement. Ajnr. 2002;23:459-67.

8. Luppino FS, Grooters E, de Bruine FT, Zwinderman AH, van der Mey AG. Volumetrical measurements in vestibular schwannoma, the influence of slice thickness and patient's repositioning. Otol Neurotol. 2006;27:962-8.

9. Rosenberg SI. Natural history of acoustic neuromas. Laryngoscope. 2000;110: 497-508.

10. Fiirgaard B, Pedersen CB, Lundorf E. The size of acoustic neuromas: CT and MRI. Neuroradiology. 1997;39:599-601.

11. Charabi S, Thomsen J, Mantoni M, Charabi B, Jorgensen B, Borgesen SE, Gyldensted C, Tos M. Acoustic neuroma (vestibular schwannoma): growth and surgical and nonsurgical consequences of the wait-and-see policy. Otolaryngol Head Neck Surg. 1995;113:5-14.

12. Stangerup SE, Caye-Thomasen $P$, Tos $M$, Thomsen J. The natural history of vestibular schwannoma. Otol Neurotol. 2006;27:547-52.

13. Charabi S, Tos M, Thomsen J, Charabi B, Mantoni M. Vestibular schwannoma growth: the continuing controversy. Laryngoscope. 2000;110:1720-5.

14. Walsh RM, Bath AP, Bance ML, Keller A, Tator $\mathrm{CH}$, Rutka JA. The role of conservative management of vestibular schwannomas. Clin Otolaryngol Allied Sci. 2000;25:28-39.

15. Silverstein $\mathrm{H}$, Schuknecht HF. Biochemical studies of inner ear fluid in man. Changes in otosclerosis, Meniere's disease, and acoustic neuroma. Arch Otolaryngol. 1966;84:395-402.

16. Pialoux P, Freyss G, Trotoux J. [Study of the physico-chemical components of the labyrinthine fluids in diseases of the inner ear. Diagnostic value. 1st results]. Ann Otolaryngol Chir Cervicofac. 1972;89:385-96.

17. O'Connor AF, Luxon LM, Shortman RC, Thompson EJ, Morrison AW. Electrophoretic separation and identification of perilymph proteins in cases of acoustic neuroma. Acta Otolaryngol. 1982;93:195-200.

18. Johnsson LG, Hawkins JE, Jr., Rouse RC. Sensorineural and vascular changes in an ear with acoustic neurinoma. Am J Otolaryngol. 1984;5:49-59. 
19. Somers T, Casselman J, de Ceulaer G, Govaerts P, Offeciers E. Prognostic value of magnetic resonance imaging findings in hearing preservation surgery for vestibular schwannoma. Otol Neurotol. 2001;22:87-94.

20. Stangerup SE, Tos M, Thomsen J, Caye-Thomasen P. Hearing outcomes of vestibular schwannoma patients managed with 'wait and scan': predictive value of hearing level at diagnosis. The Journal of laryngology and otology. 1-5.

21. Williams JA. Fractionated stereotactic radiotherapy for acoustic neuromas: preservation of function versus size. J Clin Neurosci. 2003;10:48-52.

22. Spiegelmann R, Lidar Z, Gofman J, Alezra D, Hadani M, Pfeffer R. Linear accelerator radiosurgery for vestibular schwannoma. J Neurosurg. 2001;94:7-13.

23. Rutten I, Baumert BG, Seidel L, Kotolenko S, Collignon J, Kaschten B, Albert A, Martin D, Deneufbourg JM, Demanez JP, Stevenaert A. Long-term follow-up reveals low toxicity of radiosurgery for vestibular schwannoma. Radiother Oncol. 2007;82:83-9.

24. Combs SE, Volk S, Schulz-Ertner D, Huber PE, Thilmann C, Debus J. Management of acoustic neuromas with fractionated stereotactic radiotherapy (FSRT): long-term results in 106 patients treated in a single institution. Int J Radiat Oncol Biol Phys. 2005;63:75-81.

25. Okunaga T, Matsuo T, Hayashi N, Hayashi Y, Shabani HK, Kaminogo M, Ochi M, Nagata I. Linear accelerator radiosurgery for vestibular schwannoma: measuring tumor volume changes on serial three-dimensional spoiled gradient-echo magnetic resonance images. J Neurosurg. 2005;103:53-8.

26. Meijer OW, Weijmans EJ, Knol DL, Slotman BJ, Barkhof F, Vandertop WP, Castelijns JA. Tumor-volume changes after radiosurgery for vestibular schwannoma: implications for follow-up MR imaging protocol. Ajnr. 2008;29: 906-10.

27. Zhang X, Fei Z, Chen YJ, Fu LA, Zhang JN, Liu WP, He XS, Jiang XF. Facial nerve function after excision of large acoustic neuromas via the suboccipital retrosigmoid approach. J Clin Neurosci. 2005;12:405-8.

28. Wiet RJ, Mamikoglu B, Odom L, Hoistad DL. Long-term results of the first 500 cases of acoustic neuroma surgery. Otolaryngol Head Neck Surg. 2001;124: 645-51.

29. Samii M, Gerganov VM, Samii A. Functional outcome after complete surgical removal of giant vestibular schwannomas. J Neurosurg. 112:860-7.

30. Mamikoglu B, Wiet RJ, Esquivel CR. Translabyrinthine approach for the management of large and giant vestibular schwannomas. Otol Neurotol. 2002;23:224-7.

31. Lanman TH, Brackmann DE, Hitselberger WE, Subin B. Report of 190 consecutive cases of large acoustic tumors (vestibular schwannoma) removed via the translabyrinthine approach. J Neurosurg. 1999;90:617-23.

32. Jung S, Kang SS, Kim TS, Kim HJ, Jeong SK, Kim SC, Lee JK, Kim JH, Kim SH, Lee $\mathrm{JH}$. Current surgical results of retrosigmoid approach in extralarge vestibular schwannomas. Surg Neurol. 2000;53:370-7; discussion 7-8.

33. Briggs RJ, Luxford WM, Atkins JS, Jr., Hitselberger WE. Translabyrinthine removal of large acoustic neuromas. Neurosurgery. 1994;34:785-90; discussion 90-1.

34. Lalwani AK, Butt FY, Jackler RK, Pitts LH, Yingling CD. Facial nerve outcome after acoustic neuroma surgery: a study from the era of cranial nerve monitoring. Otolaryngol Head Neck Surg. 1994;111:561-70. 
35. Lee J, Fung K, Lownie SP, Parnes LS. Assessing impairment and disability of facial paralysis in patients with vestibular schwannoma. Archives of otolaryngology-head \& neck surgery. 2007;133:56-60.

36. Cross T, Sheard CE, Garrud P, Nikolopoulos TP, O'Donoghue GM. Impact of facial paralysis on patients with acoustic neuroma. Laryngoscope. 2000;110:1539-42.

37. Sanna M, Falcioni M, Taibah A, De Donato G, Russo A, Piccirillo E. Treatment of residual vestibular schwannoma. Otol Neurotol. 2002;23:980-7.

38. Ramina R, Coelho Neto M, Bordignon KC, Mattei T, Clemente R, Pires Aguiar PH. Treatment of large and giant residual and recurrent vestibular schwannomas. Skull Base. 2007;17:109-17.

39. Pollock BE, Lunsford LD, Flickinger JC, Clyde BL, Kondziolka D. Vestibular schwannoma management. Part I. Failed microsurgery and the role of delayed stereotactic radiosurgery. J Neurosurg. 1998;89:944-8.

40. Ohta S, Yokoyama T, Nishizawa S, Uemura K. Regrowth of the residual tumour after acoustic neurinoma surgery. Br J Neurosurg. 1998;12:419-22.

41. Kameyama S, Tanaka R, Kawaguchi T, Honda $Y$, Yamazaki $H$, Hasegawa A. Long-term follow-up of the residual intracanalicular tumours after subtotal removal of acoustic neurinomas. Acta Neurochir (Wien). 1996;138:206-9.

42. Kameyama S, Tanaka R, Honda Y, Hasegawa A, Yamazaki H, Kawaguchi T. The long-term growth rate of residual acoustic neurinomas. Acta Neurochir (Wien). 1994;129:127-30.

43. Godefroy WP, van der Mey AG, de Bruine FT, Hoekstra ER, Malessy MJ. Surgery for large vestibular schwannoma: residual tumor and outcome. Otol Neurotol. 2009;30:629-34.

44. El-Kashlan HK, Zeitoun H, Arts HA, Hoff JT, Telian SA. Recurrence of acoustic neuroma after incomplete resection. Am J Otol. 2000;21:389-92.

45. Bloch DC, Oghalai JS, Jackler RK, Osofsky M, Pitts LH. The fate of the tumor remnant after less-than-complete acoustic neuroma resection. Otolaryngol Head Neck Surg. 2004;130:104-12.

46. Lunsford LD, Niranjan A, Flickinger JC, Maitz A, Kondziolka D. Radiosurgery of vestibular schwannomas: summary of experience in 829 cases. J Neurosurg. 2005;102 Suppl:195-9.

47. Kondziolka D, Lunsford LD, McLaughlin MR, Flickinger JC. Long-term outcomes after radiosurgery for acoustic neuromas. N Engl J Med. 1998;339:1426-33.

48. Chung WY, Liu KD, Shiau CY, Wu HM, Wang LW, Guo WY, Ho DM, Pan DH. Gamma knife surgery for vestibular schwannoma: 10-year experience of 195 cases. J Neurosurg. 2005;102 Suppl:87-96.

49. Park CK, Jung HW, Kim JE, Son YJ, Paek SH, Kim DG. Therapeutic strategy for large vestibular schwannomas. J Neurooncol. 2006;77:167-71.

50. Yang HC, Kano H, Awan NR, Lunsford LD, Niranjan A, Flickinger JC, Novotny J, Jr., Bhatnagar JP, Kondziolka D. Gamma Knife radiosurgery for larger-volume vestibular schwannomas. Clinical article. J Neurosurg. 114:801-7.

51. Prasad D, Steiner M, Steiner L. Gamma surgery for vestibular schwannoma. J Neurosurg. 2000;92:745-59.

52. Mandl ES, Meijer OW, Slotman BJ, Vandertop WP, Peerdeman SM. Stereotactic radiation therapy for large vestibular schwannomas. Radiother Oncol. 95:94-8.

53. Litvack ZN, Noren G, Chougule PB, Zheng Z. Preservation of functional hearing after gamma knife surgery for vestibular schwannoma. Neurosurg Focus. 2003;14:e3. 
54. Lederman G, Lowry J, Wertheim S, Fine M, Lombardi E, Wronski M, Arbit E. Acoustic neuroma: potential benefits of fractionated stereotactic radiosurgery. Stereotact Funct Neurosurg. 1997;69:175-82.

55. Inoue HK. Low-dose radiosurgery for large vestibular schwannomas: long-term results of functional preservation. J Neurosurg. 2005;102 Suppl:111-3.

56. Samii M, Gerganov V, Samii A. Improved preservation of hearing and facial nerve function in vestibular schwannoma surgery via the retrosigmoid approach in a series of 200 patients. J Neurosurg. 2006;105:527-35.

57. Post KD, Eisenberg MB, Catalano PJ. Hearing preservation in vestibular schwannoma surgery: what factors influence outcome? J Neurosurg. 1995;83: 191-6.

58. Hecht CS, Honrubia VF, Wiet RJ, Sims HS. Hearing preservation after acoustic neuroma resection with tumor size used as a clinical prognosticator. Laryngoscope. 1997;107:1122-6.

59. Fischer G, Fischer C, Remond J. Hearing preservation in acoustic neurinoma surgery. J Neurosurg. 1992;76:910-7.

60. Rowe JG, Radatz MW, Walton L, Hampshire A, Seaman S, Kemeny AA. Gamma knife stereotactic radiosurgery for unilateral acoustic neuromas. J Neurol Neurosurg Psychiatry. 2003;74:1536-42.

61. Hasegawa T, Fujitani S, Katsumata S, Kida Y, Yoshimoto M, Koike J. Stereotactic radiosurgery for vestibular schwannomas: analysis of 317 patients followed more than 5 years. Neurosurgery. 2005;57:257-65; discussion -65.

62. Yomo S, Arkha Y, Delsanti C, Roche PH, Thomassin JM, Regis J. Repeat gamma knife surgery for regrowth of vestibular schwannomas. Neurosurgery. 2009;64:4854; discussion -5 .

63. Liscak R, Vladyka V, Urgosik D, Simonova G, Vymazal J. Repeated treatment of vestibular schwannomas after gamma knife radiosurgery. Acta Neurochir (Wien). 2009;151:317-24; discussion 24

64. Pollock BE, Lunsford LD, Kondziolka D, Sekula R, Subach BR, Foote RL, Flickinger JC. Vestibular schwannoma management. Part II. Failed radiosurgery and the role of delayed microsurgery. J Neurosurg. 1998;89:949-55

65. Lee DJ, Westra WH, Staecker H, Long D, Niparko JK, Slattery WH, 3rd. Clinical and histopathologic features of recurrent vestibular schwannoma (acoustic neuroma) after stereotactic radiosurgery. Otol Neurotol. 2003;24:650-60; discussion 60.

66. Shuto $\mathrm{T}$, Inomori $\mathrm{S}$, Matsunaga $\mathrm{S}$, Fujino $\mathrm{H}$. Microsurgery for vestibular schwannoma after gamma knife radiosurgery. Acta Neurochir (Wien). 2008;150:229-34; discussion 34.

67. Friedman RA, Brackmann DE, Hitselberger WE, Schwartz MS, Iqbal Z, Berliner KI. Surgical salvage after failed irradiation for vestibular schwannoma. Laryngoscope. 2005;115:1827-32

68. Limb CJ, Long DM, Niparko JK. Acoustic neuromas after failed radiation therapy: challenges of surgical salvage. Laryngoscope. 2005;115:93-8

69. Slattery $\mathrm{WH}, 3 \mathrm{rd}$. Microsurgery after radiosurgery or radiotherapy for vestibular schwannomas. Otolaryngol Clin North Am. 2009;42:707-15

70. Friedman RA, Berliner KI, Bassim M, Ursick J, Slattery WH 3rd, Schwartz MS, Brackmann DE. A paradigm shift in salvage surgery for radiated vestibular schwannoma. Otol Neurotol 2011; 32:1322-28 

SUMMARY 


\section{Summary}

Vestibular schwannomas (VS) are benign neoplasms, originating from the Schwann cells of the vestibular part of the 8th cranial nerve. The most common symptoms, present in a patient with a VS, are unilateral sensorineural hearing loss, tinnitus, imbalance, and vertigo. An increase in VS size will result in more severe symptoms, consisting, for example, of an increased spectrum of involved cranial nerves, hydrocephalus, symptomatic mass effect due to mechanical compression of the brain stem, or ventricular obstruction.

Historically, VS were treated by means of a microsurgical resection. In recent decades there have been enormous improvements in the development of treatment options. With the introduction of MRI, more insight has been gained into the slow or absent growth pattern of the VS, justifying a wait and scan policy (W\&S). In this policy, patients are monitored by sequential MRI and clinical evaluation. In addition, radiosurgery has become increasingly popular. The latter treatment option is now generally accepted as the treatment of choice in small-medium sized progressive VS, thereby avoiding an invasive skull base procedure.

Nowadays, the treatment of the vast majority of VS does not involve a life saving procedure. Clinicians are dealing with smaller neoplasms causing little symptomatology. The main focus is on saving the function of a patient, with minimal morbidity.

This thesis evaluates new developments in both conservative and invasive therapeutic treatment options for VS patients, in which current "common practices" are compared with new ones.

Chapter 1 gives a general introduction and a review of the treatment options for VS. In addition, the aims and outline of the present thesis are described.

Chapter 2 compares two measurement techniques to evaluate the VS size and growth: the conventional two-dimensional (2D) measurements, and volume measurements. The baseline MRIs (both on contrast enhanced T1-weighted images (CE T1-WI) and on T2-weighted images (T2-WI)) of 68 consecutive VS patients were analyzed by two readers. The reproducibility of each technique was calculated, consisting of the interobserver agreement and reliability. From the interobserver agreement, we calculated the measurement error of each measuring technique. Both $2 \mathrm{D}$ and volume measurements showed best reproducibility on CE T1-WI and, in this context, volume measurements were superior compared to $2 \mathrm{D}$ measurements. A relative measurement error of $40 \%$ was seen in 2D measurements, compared to $19.7 \%$ in volume measurements. In the smallest, intracanalicular VS, a similar reproducibility was obtained for both $2 \mathrm{D}$ and volume measurements, making both modalities suitable for followup (FU) analysis. In larger VS, volume measurements were more reproducible. 
The absolute measurement error for 2D measurements varied from $2.12-2.98$ millimeter, exceeding the clinically applied arbitrary criterion of 1 or 2 millimeters for growth. Thus, evaluation of VS growth is better performed with volume measurements. If $2 \mathrm{D}$ measurements are used, the current criterion for growth should be exercised with caution.

Chapter 3 analyses risk factors for VS growth or hearing deterioration during FU in a W\&S policy. Data from 36 patients were analyzed retrospectively, with a median FU of 20 months. The risk factors were either patient-related (symptoms, age, sex) or VS-related (volume, the presence of growth, Koos gradation, labyrinthine signal intensity on T2-WI). Vestibular schwannoma evaluation was performed by volume measurements, with the corresponding measurement error, in order to determine whether or not there was VS growth. Regarding growth, no baseline risk factor was found. Growth during FU was significantly related to further VS growth. Regarding hearing deterioration, a complaint of hearing loss at diagnosis, or a hypointense labyrinthine signal intensity on $\mathrm{T} 2-\mathrm{Wl}$ at diagnosis, were significantly related to further hearing loss on pure tone audiogram during FU. These findings can aid clinicians dealing with VS patients in a W\&S policy.

Chapter 4 retrospectively evaluates 37 patients, who were treated between 2003 and 2008 with linear accelerator (LINAC) based stereotactic radiosurgery or fractionated radiotherapy because of a progressive small-medium sized VS, by using the validated volumetric measuring tool. Also, growth patterns, and risk factors predicting treatment failure were analyzed. The median FU was 40 months. A 4-year probability of radiological growth control was seen in $85.4 \%$ of the cases; clinical control (absence of additional intervention) was seen in $96.4 \%$. This indicates that radiological growth was not considerable enough, in many patients, to undergo a second VS-related intervention. Overall, shrinkage was seen in $65 \%$, stable VS in $22 \%$, and growth in $13 \%$. In $54 \%$ of all patients transient swelling was observed, which could last long after treatment (median time until regression was 24 months (range 11-62). This should not be regarded as continuoing growth or treatment failure. No prognostic factors were found regarding VS growth. Previous treatment or treatment with radiosurgery were significantly associated with transient swelling. The lower amount of radiological growth control is attributed to the use of the more sensitive volume measurements. Review of the literature reveals a median growth control of $96 \%$ in studies measuring with 2D measurements and $90 \%$ in studies using volume measurements. Clinical control however, was similar between these studies (median 97\%), and compared well with the present study.

Chapter 5 retrospectively analyses a group of 50 patients with a large VS who were treated first with a subtotal microsurgical resection, followed by Gamma Knife surgery (GKS) of the residual tumor, between 2002 and 2009. Surgery was performed with a translabyrinthine (25 patients) or retrosigmoid (25 
patients) approach. Vestibular schwannoma measurements were calculated volumetrically. The mean preoperative VS volume was $14.9 \mathrm{~cm}^{3}$; the postoperative volume was $3.3 \mathrm{~cm}^{3}$.

Patient charts were reviewed in order to analyze clinical symptoms accompanying the large VS, and after the treatment. Audiograms were evaluated in order to classify hearing pre- and postoperatively. After a median FU of 33.8 months, a radiological growth control was seen in $90 \%$ of the patients, and clinical control in $92 \%$. Good facial nerve function (HouseBrackmann Grade I or II) was present one year after GKS in $94 \%$ of the cases. One of the two patients receiving hearing-preserving surgery maintained serviceable hearing during 37 months of FU. One major complication (a hemiparesis) occurred in a patient receiving re-intervention after subtotal resection because of hematoma formation due to a coagulation disorder. Four patients needed a second intervention, three received second GKS and the fourth patient needed second microsurgical resection, complicated by a facial nerve paralysis. During further FU, growth control was seen here. The subtotal resection followed by GKS can be a treatment option of choice in large VS.

Chapter 6 investigates the effect of primary GKS on large VS by evaluating retrospectively 33 patients treated between 2002 and 2009. A large VS was defined as a VS $>6 \mathrm{~cm}^{3}$, at least indenting the brain stem. The choice for primary GKS was based on the presence of serviceable hearing (12 patients), patient's choice (17 patients) or co-morbidity (4 patients). The mean VS volume prior to GKS was $8.8 \mathrm{~cm}^{3}$. As with the study reported in the previous chapter, VS measurements were calculated volumetrically and patient charts were reviewed in order to analyze clinical symptoms accompanying the large VS, and after the GKS treatment. Audiograms were evaluated in order to classify hearing pre- and postoperatively. After a median FU of 30 months, a radiological growth control was seen in $88 \%$ of the patients, clinical control was seen in $79 \%$ of the patients. No major complications occurred. During a FU of 37 months $58 \%$ of the patients entering GKS with serviceable hearing, maintained this hearing. Preservation of the facial and trigeminal nerves was achieved in $91 \%$ and $86 \%$ of patients respectively; in all of these cases the nerve dysfunction was transient. Seven patients needed secondary intervention because of either severe symptoms (1 patient) or growth (not always beyond baseline volume, therefore not always detected as radiological growth control failure) with minimal or absent symptoms (6 patients). Four patients received a subtotal microsurgical resection, followed by GKS. In one of these patients a facial nerve paralysis occurred. Two patients received a second GKS treatment. In all patients growth control was achieved during further FU. Primary GKS for large VS, therefore, leads to acceptable radiological and clinical control rates, with the chance to preserve functional hearing. One should take the greater number of clinical failures into account, compared to 
the results in Chapter 5. This shows that there is little reserve to observe growth or swelling in these large VS by means of imaging, when performing primary GKS, necessitating a second intervention.

Chapter 7 reports the editorial written by experts in the field of VS management: dr Kondziolka and Bambakidis et al., and the response to this editorial by van de Langenberg et al.

Chapter 8 comprises the general discussion and the conclusion of the results described in the thesis. Moreover, future perspectives are discussed.

Finally, we can conclude that the current management of VS regarding the measuring technique and the criterion for growth is outdated. Because of the increase in user-friendly volume measuring software, it is to be expected that volume measurements will replace $2 \mathrm{D}$ measurement, for non-intracanalicular VS, both in a W\&S policy and after intervention. The criterion for growth used thus far should now be used with caution. Furthermore, it has been shown that VS growth at the time of diagnosis is not predictable, although growth during the first year of FU predicts further growth. Hearing loss or a labyrinthine hypointensity on T2-WI at diagnosis, predict further hearing loss during FU. Moreover, volume measurements are more sensitive to detection of growth after radiosurgery, which will result in a level of radiological growth control lower than those reported in studies performing 2D measurements. This diminished radiological growth rate does not have clinical consequences in small-medium sized VS. In addition, the treatment options for VS continue to evolve; treatment techniques which are not yet in widespread use are described that further diminish the post therapeutic morbidity in patients with a large VS. These should further improve the perspectives of VS patients. 
SAMENVATTING 


\section{Samenvatting}

Een vestibulair schwannoom (VS) is een goedaardige zenuwschede tumor uitgaande van het vestibulaire deel van de $8^{\text {ste }}$ hersenzenuw. Deze tumoren gaan uit van de Schwann cellen. De meest voorkomende symptomen bij een patiënt met een VS zijn unilateraal perceptief gehoorverlies, tinnitus, dysbalans en duizeligheid. Indien een VS progressie toont kunnen ernstigere symptomen ontstaan. Dit kan zich uiten in dysfunctie van meerdere hersenzenuwen of symptomatisch massa effect ten gevolge van mechanische compressie van het VS op de hersenstam met eventueel het ontstaan van een hydrocephalus.

In het verleden werden VS vooral microchirurgisch behandeld. Het is echter gebleken, na de introductie van de MRI, dat VS vaak langzaam of niet groeien. Dit typische groeikarakter rechtvaardigt een "wait and scan" beleid. Deze patienten worden periodiek gecontroleerd middels een MRI, in combinatie met een poliklinische follow-up (FU). Deze groep patiënten blijft een interventie bespaard. Indien er toch sprake is van VS groei, wordt in toenemende mate radiochirurgie toegepast. Hiermee kan een operatieve schedelbasisingreep worden vermeden. Voor het grootste deel van de kleine en middelgrote progressieve VS is dit tegenwoordig de behandelingsvorm van eerste keuze.

De behandeling van een VS betrof decennia geleden voornamelijk een levensreddende procedure. Heden ten dage worden VS veelal eerder gediagnosticeerd, waarbij tijdens behandeling, het functiebehoud centraal staat.

In dit proefschrift worden nieuwe ontwikkelingen in zowel conservatieve als invasieve behandelingsopties voor VS nader bekeken. Ook worden deze vergeleken met de huidige klinische praktijk.

In Hoofdstuk 1 wordt een algemene introductie gegeven met een review van de verschillende behandelingsopties voor VS. Tevens wordt het doel van de verschillende studies behandeld en worden de hoofdlijnen van dit proefschrift beschreven.

In Hoofdstuk 2 worden twee meetmethoden, die gebruikt worden om de grootte en eventuele groei van het VS te bepalen, met elkaar vergeleken. Dit betreffen de conventionele twee dimensionale (2D) en volumemetingen. Voor dit onderzoek werden de baseline MRIs van 68 patiënten geanalyseerd door twee mensen met ervaring in het opmeten van VS. Het betroffen zowel contrast gekleurde, T1-gewogen opnamen (CE T1-WI) en T2-gewogen opnamen. De reproduceerbaarheid van elke techniek werd berekend. De reproduceerbaarheid bestaat uit de "overeenkomstigheid" tussen twee readers (de "interobserver agreement", hieruit kunnen we de meetfout van een bepaalde meetmethode berekenen) en de "betrouwbaarheid" (de "reliability") van een meetmethode. Zowel de $2 \mathrm{D}$, als de volumemetingen gaven een betere 
reproduceerbaarheid op de CE T1-WI, en in deze setting waren de volumemetingen superieur vergeleken met de $2 \mathrm{D}$ metingen. Een relatieve meetfout van $40 \%$ werd gezien bij de 2D metingen, vergeleken met $19,7 \%$ bij de volumemetingen. Bij de kleinste VS, dat zijn de intracanaliculair gelegen VS, werd eenzelfde reproduceerbaarheid gevonden voor beide meetmethoden: zowel de $2 \mathrm{D}$ als de volumemetingen kunnen gebruikt worden voor metingen bij deze VS. Bij grotere VS behaalden de volumemetingen een betere reproduceerbaarheid. De maximale absolute meetfout voor 2D metingen varieerde van 2.12 tot 2.98 millimeter. Dit is meer dan de in de kliniek gehanteerde arbitraire grens van 1 of 2 millimeter voor VS groei. Het evalueren van eventuele VS groei, kan preciezer verricht worden met volumemetingen. Wanneer toch de 2D metingen gebruikt worden, dan dient het huidige criterium voor VS groei met terughoudendheid gebruikt te worden.

In Hoofdstuk 3 worden risicofactoren bekeken, welke VS groei of gehoorverlies gedurende FU kunnen voorspellen wanneer deze VS conservatief vervolgd worden in een wait and scan beleid. De data van 36 patiënten werd retrospectief bekeken, de mediane FU duur bedroeg 20 maanden. De risicofactoren waren zowel patiënt gerelateerd (symptoom, leeftijd, geslacht) als VS gerelateerd (VS volume, aanwezigheid van groei, Koos gradatie, signaal intensiteit van het labyrinth op T2-gewogen opnamen). VS metingen werden verricht met behulp van volumemetingen, met de bij deze software en images behorende meetfout, om te bekijken of er sprake was van VS groei. Voor het voorspellen van VS groei, konden geen baseline factoren geïdentificeerd worden. Wel was groei gedurende het eerste jaar van de FU, significant gerelateerd aan verdere groei gedurende de FU. Voor wat betreft het gehoorverlies gedurende FU werden twee risicofactoren geïdentificeerd: mensen met gehoorverlies ten tijde van diagnose of een hypointens labyrinth op T2-gewogen opnamen lieten significant meer gehoorverlies zien op het toonaudiogram, vergeleken met niet aangedane labyrinthen. Deze bevindingen kunnen de clinici helpen die VS patiënten vervolgen in een wait and scan beleid.

Hoofdstuk 4 betreft een retrospectieve studie, waarin 37 mensen gevolgd worden die tussen 2003 en 2008 zijn behandeld met lineair geaccelereerde (LINAC) radiochirurgie in verband met een progressief klein-middelgroot VS. De VS metingen werden verricht met behulp van gevalideerde volume software. Ook werden groeipatronen bekeken en risicofactoren, welke het falen van de behandeling zouden kunnen voorspellen. De mediane FU duur was 40 maanden. De 4 jaars waarschijnlijkheid voor het verkrijgen van radiologische groei controle en klinische controle (afwezigheid van een tweede interventie) werd gezien in respectievelijk $85.4 \%$ en $96.4 \%$ van de gevallen. Dit laat zien dat niet alle radiologische groei aanzienlijk genoeg was om een tweede VS interventie te ondergaan. Een daling van het VS volume werd gezien in $65 \%$ 
van de gevallen, in $22 \%$ was er sprake van een stabiele tumor, en in $13 \%$ van de gevallen werd VS groei gezien. In $54 \%$ van de patiënten werd tijdelijke tumor zwelling gezien. Dit kon tot lang na behandeling aanwezig zijn (mediane tijdsduur tot regressie van tijdelijke zwelling was 24 maanden (range 11-62). Dit moet niet gezien worden als continue groei of falen van de behandeling. Geen factoren werden gevonden, die vooraf VS groei zouden kunnen voorspellen. Een behandeling voorafgaande aan de LINAC behandeling, evenals behandeling middels radiochirurgie was significant gerelateerd aan het optreden van tijdelijke tumor zwelling. De verminderde radiologische groeicontrole wordt gewijd aan het gebruik van de meer sensitieve volumemetingen. Een review van de literatuur laat een mediane groeicontrole van $96 \%$ in studies welke 2D VS meten na radiochirurgie en $90 \%$ in studies welke volumemetingen gebruiken. De klinische controle was echter gelijk in deze studies (mediane controle van 97\%), wat hetzelfde is als in de huidige studie.

In Hoofdstuk 5 wordt een groep van 50 patiënten met een groot VS nader bekeken, die eerst behandeld zijn middels een subtotale microchirurgische resectie, gevolgd door Gamma Knife chirurgie (GKS) van het tumor restant. Het betreft een retro-spectieve analyse van de periode 2002 - 2009. De VS werden translabyrinthair (25 patiënten) en retrosigmoidaal (25 patiënten) benaderd. Het gemiddelde pre-operatieve VS volume was $14.9 \mathrm{~cm}^{3}$, het postoperatieve volume was $3.3 \mathrm{~cm}^{3}$.

Vestibulair schwannoom volumemetingen werden uitgevoerd met behulp van speciale volume software. De statussen werden bekeken om de kliniek te achterhalen van deze grote VS bij presentatie en gedurende de periode na de behandeling. Audiogrammen werden geanalyseerd om inzicht te krijgen in het gehoor voor en na de behandeling. Na een mediane FU duur van 33.8 maanden, werd een radiologische groei controle gezien van $90 \%$ van de patiënten en een klinische controle van $92 \%$. Een jaar na GKS behandeling werd een goede functie van de nervus facialis (House Brackmann Graad I of II) gezien in $94 \%$ van de patiënten. Een van de twee patiënten welke gehoorsparende chirurgie had ondergaan, behield dit gehoor gedurende 37 maanden FU. Een ernstige complicatie (hemiparese) trad op bij een patiënt die een tweede ingreep nodig had in verband met hematoomvorming bij een stollingsstoornis. Vier patiënten hadden een tweede behandeling nodig, 3 hiervan ondergingen een tweede GKS behandeling, en de vierde patiënt onderging een tweede microchirurgische benadering van de VS rest. Dit werd gecompliceerd door een facialis paralyse. Gedurende verdere FU werd groei controle gezien in deze groep. De subtotale VS resectie, gevolgd door GKS kan een behandelingsoptie van keus zijn bij grote VS.

Hoofdstuk 6 beschrijft het beloop van 33 patiënten met een groot VS, welke allen behandeld zijn met primaire GKS, tussen 2002 en 2009. Het betreft een retrospectieve studie. Een groot VS werd gedefinieerd als een VS $>6 \mathrm{~cm}^{3}$, die 
op zijn minst de hersenstam raakte. De keuze voor primaire GKS was gebaseerd op het aanwezig zijn van een functioneel gehoor (12 patiënten), specifieke keuze van de patiënt (17 patiënten) of comorbiditeit (4 patiënten). Het gemiddelde VS volume voor GKS was $8.8 \mathrm{~cm}^{3}$. Vestibulair schwannoom metingen werden verricht met behulp van speciale volume software. De statussen werden doorgenomen om de kliniek te bestuderen voor en na de GKS behandeling. Audiogrammen werden geanalyseerd om het gehoor voor en na de behandeling te bekijken. $\mathrm{Na}$ een mediane FU duur van 30 maanden werd een radiologische groei controle gezien in $88 \%$ van de gevallen, een klinische controle in $79 \%$ van de gevallen. Er traden geen grote complicaties op. Gedurende een mediane FU van 37 maanden behield $58 \%$ van de patiënten die zich voor behandeling presenteerden met een functioneel gehoor, dit gehoor. Behoud van de functie van de nervus facialis werd gezien in respectievelijk $91 \%$ en $86 \%$, waarbij deze dysfunctie in alle gevallen slechts van tijdelijke aard was. Zeven patiënten moesten een tweede ingreep ondergaan ten gevolge van ernstige symptomen (1 patiënt) en groei (niet altijd boven baseline volume en daarom niet altijd gedetecteerd als falen van radiologische groei controle) met minimale of geen symptomen (6 patiënten). Vier patiënten ondergingen een subtotale resectie, gevolgd door GKS. In een van deze patiënten trad een nervus facialis paralyse op. Twee patiënten ontvinden een tweede GKS behandeling. In alle patiënten die een tweede behandeling ondergingen trad radiologische groei controle op. Primaire GKS leidt tot acceptabele radiologische groei en klinische controle met een kans op behoud van functioneel gehoor. Het grotere aantal klinische failures, vergeleken met de resultaten in hoofdstuk 5 , laat zien dat er weinig reserve is om tijdelijke zwelling of vervolg groei van het VS af te wachten.

Hoofdstuk 7 is een editorial, geschreven door experts op het gebied van VS management: Kondziolka en Bambakidis et al., en de reactie hierop door van de Langenberg et al.

In Hoofdstuk 8 worden zowel de algemene discussie als de conclusies van het proefschrift op een rij gezet. Ook worden toekomstperspectieven en voorstellen voor toekomstig VS-gerelateerd onderzoek beschreven.

Samenvattend, kunnen we concluderen dat de huidige manier van het meten van de grootte en eventuele groei van het VS, verouderd is. Omdat er steeds meer gebruiksvriendelijke software op de markt verschijnt, ligt het in de lijn der verwachting dat volumemetingen op termijn de 2D metingen gaan vervangen. Dit geldt voor de niet intracanaliculair gelegen VS, zowel in een wait and scan beleid als na interventie. Tot het zover is, dient de arbitraire grens voor groei, zoals deze nu gebruikt wordt, met terughoudendheid toegepast worden. Naast deze bevindingen hebben we aangetoond dat groei van het VS ten tijde van diagnosestelling niet voorspelbaar is, hoewel groei gedurende het eerste jaar 
van de FU verdere groei voorspelt. Gehoorverlies of verlies van signaal intensiteit van het labyrinth op T2-gewogen MRI opnames ten tijde van de diagnosestelling, voorspelt verder gehoorverlies gedurende de FU. Volumemetingen zijn sensitiever in het detecteren van groei van het VS. Na een radiochirurgische behandeling zal het percentage van VS, waarbij de groei radiologisch gecontroleerd is, lager uitvallen bij gebruik van volumemetingen, in vergelijking met studies die de VS 2D opmeten. Deze verminderde controle blijft zonder klinische consequenties bij de beschreven kleine-middelgrote VS. Tenslotte hebben we behandelingstechnieken beschreven voor grote VS, die op dit moment nog niet wijdverspreid gebruikt worden. Deze kunnen de morbiditeit na behandeling verder terug dringen. De perspectieven van patiënten met een VS kunnen hierdoor verder verbeteren. 

DANKWOORD 


\section{Dankwoord}

Alle mensen die op enige wijze hebben bijgedragen aan de totstandkoming van dit proefschrift wil ik hartelijk danken voor hun inzet. Er zijn bij het realiseren van het proefschrift een aantal personen betrokken die ik in het bijzonder zou willen danken voor hun bijdrage.

Prof. dr. Stokroos, beste Robert. Eind 2006 maakte ik een afspraak bij je om mijn interesse in de KNO kenbaar te maken en om te kijken of ik op wetenschappelijk gebied nog iets kon betekenen. Je had nog een leuk project liggen betreffende de imaging van vestibulair schwannomen... ik ben je enorm dankbaar voor het vertrouwen dat je vanaf het begin in me hebt gehad en de kans die je me hebt gegeven om dit onderzoek in opleidingssetting te continueren. Zonder jouw heldere ideeën, contacten en enthousiasme was dit boekje er niet gekomen.

Daarnaast dank ik prof. dr. B. Kremer, mijn tweede promotor. Beste Bernd, graag wil ik $u$ danken voor uw advies en vertrouwen gedurende het onderzoek. Als opleider bent $u$ een voorbeeld waar ik veel van leer.

Prof. dr. J.J. van Overbeeke, drs. P. Hanssens en dr. J. Verheul, beste Koo, Patrick en Jeroen. Jullie, als Gamma Knife team, wil ik hartelijk danken voor het faciliteren van en participeren in ons onderzoek naar de grote schwannomen. Patrick wil ik in het bijzonder danken voor zijn zeer meticuleuze manier van registreren.

Dr. P.J. Nelemans, beste Patty. Altijd kon ik langskomen met vragen. Je wist altijd de vinger op de zere plek te leggen. Hoewel ik menig maal met lood in mijn schoenen bij je ben vertrokken, heeft je opbouwende kritiek het geheel kwalitatief enorm verbeterd. Daarvoor ben ik je veel dank verschuldigd.

Graag wil ik de volgende co-auteurs nog bijzonder hartelijk danken: dr. B.G. Baumert, Brigitta, voor je waardevolle bijdrage aan het geheel vanuit de MAASTRO, in het bijzonder onze LINAC serie. Dr. B.J. de Bondt, Bert Jan, helaas vertrokken uit het Maastrichtse, ik dank je voor het meedenken en meewerken vanuit de neuroradiologie. Drs. A.J.C. Dohmen, Amy, dank voor je inzet als wetenschapsstudent.

De manuscriptcommissie wil ik bedanken voor hun aandachtige leeswerk.

Graag wil ik, naast mijn promotores, de overige leden van de vakgroep KNO, Janny Hof, Martin Lacko, Kenneth Kross*, Jan Wouter Brunings*, Laura 
Baijens, Jos Straetmans, Joost van Tongeren, Lucien Anteunis, Jan Brokx, Erwin George en prof. Herman Kingma, danken voor hun hulp en interesse, waar nodig. Het is erg prettig werken en opgeleid worden als er een hecht team staat.

Uiteraard wil ik de medewerkers van de afdeling KNO te Maastricht danken voor de ondersteuning tijdens het realiseren van dit proefschrift. Dat geldt ook voor de medewerkers van de afdeling KNO, Elkerliek ziekenhuis te Helmond, waar ik tijdens mijn eerste perifere stage onder begeleiding van Paul Schuil, Harm Kahmann en Martijn Alles met veel plezier heb gewerkt.

Natuurlijk dank ik ook alle (oud) assistenten KNO: Sjors van Pinxteren, Luke Schoenmakers, Job Postelmans, Jérôme Waterval ${ }^{*}$, Jeroen Mooren*, Diane Smit, Guido Dees*, Josine Widdershoven, Raymond van de Berg*, Marieke Lips, Sietze Reitsma, Nicoline Leunis, Darius Henatsch, Simone Schaefer en Sanne Wagemakers. Ik ben erg blij dat ik jullie als collega heb (gehad).

Al mijn vrienden en vriendinnen wil ik danken voor een onvergetelijke (studie) tijd. Een aantal noem ik hier in het bijzonder:

"Het HS", Maastrichts beruchtste studentenhuis, warme thuishaven van vier frivole bazen. Jan T(!)en Bosch (4), Joost van der Vorst (1), Thijs van Herpt (3) en ikzelf (2). Ontelbaar mooie herinneringen hebben we aan onze fantastische studententijd overgehouden, en nog steeds weten we elkaar te vinden. Waarom mag je maar twee paranimfen kiezen? Ondanks het feit dat het huis niet meer is, ben ik er zeker van dat deze band altijd blijft bestaan.

Verder wil ik alle (oud) leden van Onafhankelijk Heeren Dispuut Silenus danken, voor de jaren die ik nooit meer zal vergeten: stultus est cui bibere non placet! Tien jaar na dato kleurt Maastricht nog steeds bordeaux rood-oranje! Ook dank ik "groep Tilburg": ondanks het feit dat we meer dan twee lustra sinds ons vertrek uit Tilburg achter ons hebben liggen, houden we contact.

En dan mijn familie. Lieve papa en mama, Rien en Margriet. Ik wil jullie danken voor jullie onvoorwaardelijke steun en vertrouwen. Niets is teveel, altijd staan jullie voor me klaar. Zo kan ik me alleen al vijf verhuizingen binnen Maastricht herinneren... Hoewel we elkaar minder vaak zien dan voorheen, probeer ik Goirle vaak te betrekken in mijn reisplan (en dan kom ik inderdaad om weer weg te gaan (-)). Ondanks het feit dat jullie drie enorm verschillende zoons hebben gekregen vind ik het fantastisch hoe jullie ons alle drie gelijkwaardig supporten. Op deze manier heeft ieder er voor zichzelf het maximale uit kunnen halen. Ik ben jullie enorm dankbaar voor alles wat ik van jullie heb meegekregen. 
Natuurlijk dank ik ook mijn schoonfamilie, Hub en Rommie, Ruud en Sadaf, waar ik me altijd erg thuisvoel. Dankzij jullie is het Limburgs makkelijker voor mij te verstaan en kan ik met de lokale patiënten en caissières gesprekken in het dialect voeren.

Thijs en Geert, mijn broers. Ik vind het erg goed te weten dat ik in Tilburg altijd een plek heb om te crashen. Ondanks het feit dat we op veel vlakken totaal verschillen, zijn we toch beste vrienden en treffen we ons op feestjes, zomaar, of toch bij de gezamenlijke liefde: Willem II.

Verder vind ik het een eer dat oma Riki, oma Sonja en opa Piet op deze dag aanwezig kunnen zijn. Allemaal op naar de 100!

Lieve Celien. Waar een kijkavond voor een studentenkamer in Maastricht al niet goed voor is... Het gebeurt niet vaak dat een studentenrelatie zo lang stand houdt..! Gelukkig zijn wij hier een positieve uitzondering op. We begrijpen elkaar goed, en weten ons drukke leven goed te combineren met alle andere dingen die we zo graag (samen) doen! Jij bent de allerliefste, ik weet zeker dat we samen een mooie toekomst tegemoet gaan! 

CURRICULUM VITAE 


\section{Curriculum Vitae}

Rick van de Langenberg werd op 25 oktober 1983 geboren te Tilburg. In 2002 behaalde hij het eindexamen gymnasium aan het Koning Willem II college te Tilburg. Datzelfde jaar begon hij met de studie geneeskunde aan de Universiteit van Maastricht. Eind 2007 begon hij aan zijn semi-arts stage op de afdeling Keel-, Neus- en Oorheelkunde van het Maastricht Universitair Medisch Centrum (MUMC). Tijdens zijn wetenschapsstage werd de basis gelegd van het huidige promotieonderzoek. Na het behalen van het artsexamen, startte hij in december 2008 met de opleiding Keel-, Neus- en Oorheelkunde in het MUMC bij opleider Prof. dr. B. Kremer en waarnemend opleider Prof. dr. R.J. Stokroos. De eerste perifere opleidingsstage werd afgerond in het Elkerliek Ziekenhuis te Helmond, onder begeleiding van dr. P.J. Schuil en drs. H.F. Kahmann. Op 1 oktober 2012 zal hij starten met zijn tweede perifere opleidingsstage in het Catharina Ziekenhuis te Eindhoven, onder begeleiding van dr. F.C.P.M. Adriaansen en dr. D.J.M. Mateijsen. 

LIST OF PUBLICATIONS 


\section{List of publications}

\section{Papers}

van de Langenberg R, Dohmen AJ, de Bondt BJ, Nelemans PJ, Baumert BG, Stokroos RJ. Volume changes after stereotactic LINAC radiotherapy in vestibular schwannoma: control rate and growth patterns. Int J Radiat Oncol Biol Phys. 2012;84:343-49.

van de Langenberg $R$, Hanssens PE, van Overbeeke JJ, Verheul JB, Nelemans PJ, de Bondt BJ, Stokroos RJ. Response to editorials. J Neurosurg. 2011;115:898-9.

van de Langenberg R, Hanssens PE, van Overbeeke JJ, Verheul JB, Nelemans PJ, de Bondt BJ, Stokroos RJ. Management of large vestibular schwannoma. Part I. Planned subtotal resection followed by Gamma Knife surgery: radiological and clinical aspects. J Neurosurg. 2011;115:875-84.

van de Langenberg $R$, Hanssens $P E$, Verheul JB, van Overbeeke JJ, Nelemans PJ, Dohmen AJ, de Bondt BJ, Stokroos RJ. Management of large vestibular schwannoma. Part II. Primary Gamma Knife surgery: radiological and clinical aspects. J Neurosurg. 2011;115:885-93.

van de Langenberg R, de Bondt BJ, Nelemans PJ, Dohmen AJ, Baumert BG, Stokroos RJ. Predictors of volumetric growth and auditory deterioration in vestibular schwannomas followed in a wait and scan policy. Otol Neurotol. 2011;32:338-44.

van de Langenberg R, de Bondt BJ, Nelemans PJ, Baumert BG, Stokroos RJ. Follow-up assessment of vestibular schwannomas: volume quantification versus two-dimensional measurements. Neuroradiology. 2009;51:517-24.

van de Langenberg R, Stokroos R, de Bondt BJ. Radiology quiz case 2. Metastasis from NSCLC in both IACs. Arch Otolaryngol Head Neck Surg. 2009;135:717-9.

van de Langenberg R, Scheltinga MR, Streukens SA, Boelens O, Roumen RM. [Elderly women with abdominal pain due to an incarcerated 'femoral hernia']. Ned Tijdschr Geneeskd. 2008;152:1597-601. 


\section{Book contributions}

van de Langenberg R, Stokroos RJ. Vestibular schwannoma: optimizing tumor growth monitoring by volume measurements. In: Hayat MA, ed. Tumors of the central nervous system, volume 7: Meningiomas and Schwannomas. Berlin, Germany. Springer. 2012:233-8 